Portland State University

PDXScholar

Spring 6-5-2013

\title{
The Call for Cultural Responsiveness: Teachers' Perceptions about the Interplay Between Culturally Responsive Instruction and Scripted Curricula
}

Kathryn Elizabeth Toppel

Portland State University

Follow this and additional works at: https://pdxscholar.library.pdx.edu/open_access_etds

Part of the Bilingual, Multilingual, and Multicultural Education Commons, and the Educational Leadership Commons

Let us know how access to this document benefits you.

\section{Recommended Citation}

Toppel, Kathryn Elizabeth, "The Call for Cultural Responsiveness: Teachers' Perceptions about the Interplay Between Culturally Responsive Instruction and Scripted Curricula" (2013). Dissertations and Theses. Paper 1002.

https://doi.org/10.15760/etd.1002

This Dissertation is brought to you for free and open access. It has been accepted for inclusion in Dissertations and Theses by an authorized administrator of PDXScholar. Please contact us if we can make this document more accessible: pdxscholar@pdx.edu. 
The Call for Cultural Responsiveness: Teachers' Perceptions about the Interplay Between Culturally Responsive Instruction and Scripted Curricula

by

Kathryn Elizabeth Toppel

A dissertation submitted in partial fulfillment of the requirements for the degree of

\author{
Doctor of Education \\ in \\ Educational Leadership: Curriculum and Instruction
}

\begin{abstract}
Dissertation Committee:
Samuel D. Henry, Chair

Karen Noordhoff

Julie Esparza-Brown

Masami Nishishiba
\end{abstract}

Portland State University

2013 
(C)2013 Kathryn Elizabeth Toppel 


\begin{abstract}
The increased focus on the implementation of scientifically research-based instruction as an outcome of No Child Left Behind ("Understanding NCLB," 2007) has resulted in the widespread use of scripted reading curricula (Dewitz, Leahy, Jones, and Sullivan, 2010), which typically represents Eurocentric and middle class forms of discourse, knowledge, language, culture, and historical interpretations as academic knowledge (Howard, 2010; Delpit, 2012). In an era where the number of culturally and linguistically diverse students is increasing rapidly (Ginsberg, 2007), it is essential to consider that educational practices relying entirely on prefabricated content may require modification because, as recognized in the funds of knowledge theoretical framework (Veléz-Ibañez, 1988), all students bring a wealth of knowledge to the classroom that should be acknowledged, respected, valued and incorporated into instruction (Gonzalez, Moll, \& Amanti, 2005). However, even if teachers are granted the time and permission to modify scripted content in order to build bridges between the prescribed lessons and students' lived experiences, doing so is not easily accomplished when the lives of educators are disconnected from their students (Baeder, 2010).

This study investigated the behaviors and ideas teachers have developed as ways to connect with their culturally and linguistically diverse students and their families. Additionally, the study explored how teachers who implement scripted curricula describe the experience of creating culturally responsive lessons intended to specifically connect
\end{abstract}


with their culturally and linguistically diverse students and to connect with students' funds of knowledge.

This multiple case study describes how five teachers who implement scripted curricula reported their experiences of creating culturally responsive lessons for particular focal students. Findings are presented in individual case narratives followed by a crosscase synthesis. Findings suggest that teachers were able to carry out culturally responsive instructional practices while implementing scripted curricula; however, participants' CARE lessons did not represent Gay's (2010) ethnic and cultural diversity in curriculum content component of culturally responsive instruction. Additionally, findings indicate that building relationships with culturally and linguistically diverse students was key to adjusting instruction to suit their learning styles. The implications of these findings are discussed in recommendations for in-service teacher professional development and future research. 


\section{DEDICATION}

This work, and all that led up to it, is dedicated to my husband John for encouraging me to go for it and for always loving me for who I am.

Here's to the crazy ones! 


\section{ACKNOWLEDGEMENTS}

First and foremost, I would like to thank Dr. Samuel Henry for his unfaltering willingness to support me through my first year of the doctoral program via Skype as well as his gentle guidance, encouragement, and belief in my leadership capacity throughout my doctoral program. Equally important, I would like to thank Dr. Karen Noordhoff for her deep commitment to the well being of our group and for her sage leadership throughout the amazing journey. Thank you to my colleagues and cohortmates, Bernd, Edgar, Audrey, Sarah, and Jennifer. I cannot imagine this experience without them and am so thankful to have been given the gift of their inspiring presence, their astute feedback, and most importantly, their friendship. I would also like to thank my committee for their feedback and interest in my work. Finally, I would like to thank John for his love and support, Grace for her well-timed naps during her attendance at my dissertation seminars, and all my friends and family who have supported me during this journey. 
TABLE OF CONTENTS

Page

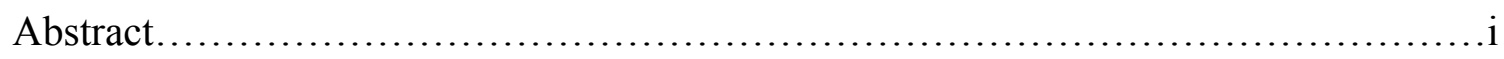

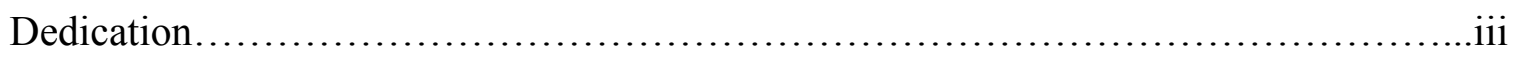

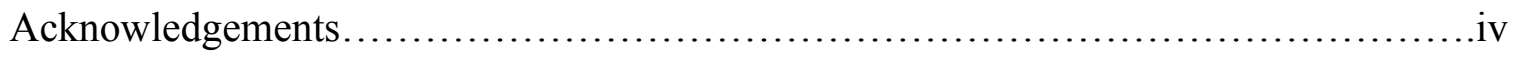

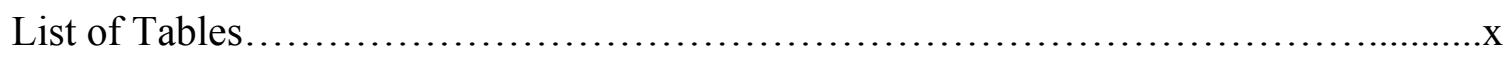

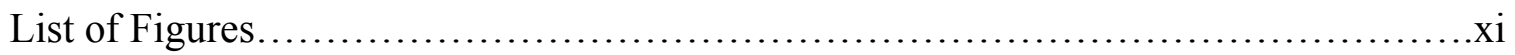

\section{CHAPTER}

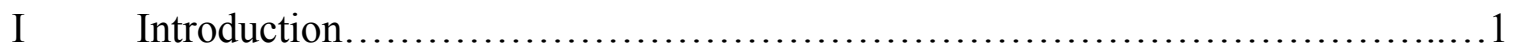

Inequalities for Culturally and Linguistically Diverse Students..................4

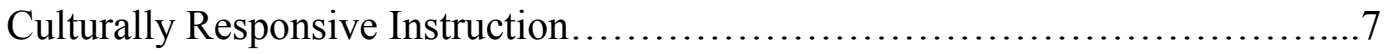

Utilizing Funds of Knowledge............................................13

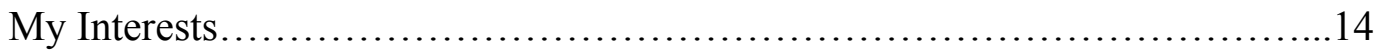

Role of the Researcher.................................................. 19

Statement of the Purpose..................................................20

Research Questions.............................................21

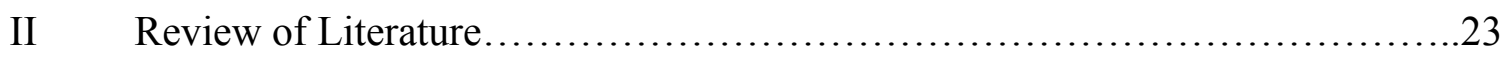

Foundations of Curriculum Theory ........................................23

Historical Background of Curriculum Debates...........................22

John Dewey-The Child and the Curriculum...........................26

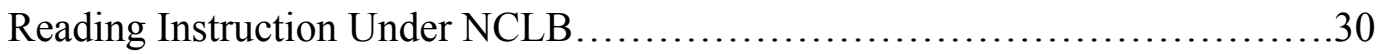


Culturally Responsive Instruction......................................31

Culturally Appropriate Texts......................................33

Engaging Student Voice......................................... 34

Incorporating Funds of Knowledge.................................35

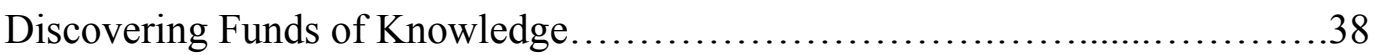

Teachers' Attitudes Before Home Visits............................42

Teachers Perspectives After Home Visits............................44

Culturally Responsive Instruction with Scripted Curricula.....................47

Conclusions.....................................................54

III Research Design and Methodology ....................................57

Research Questions.................................................57

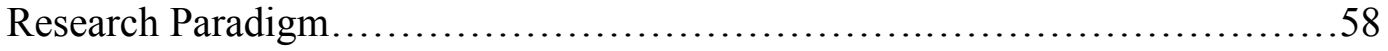

Theoretical Framework...............................................6 60

Overview of Research Methods.........................................61

Context of the Study..........................................61

Participants..................................................66

Methodological Approach.......................................68

Data Collection................................................ 71

Individual Interviews...................................... 71

Focus Group.............................................. 74

Artifacts from CARE Training............................77

Field Notes/Researcher Log..............................78

Ethical Considerations............................................ 79

Timeline and Schedule.......................................8 80

Data Analysis...............................................81 
Phase One: Within-Case Analysis.........................83

Cycle 1: Structural Coding............................84

Cycle 2: Descriptive Coding.........................85

Cycle 3: Values Coding.............................86

Reorganization of Data...............................87

Phase Two: Cross-Case Analysis...........................90

Phase Three: Assertions.....................................94

IV Results.........................................................95

Context for the Cases....................................................96

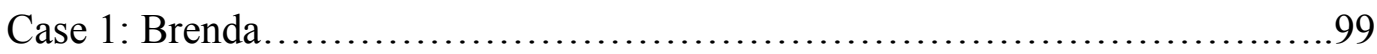

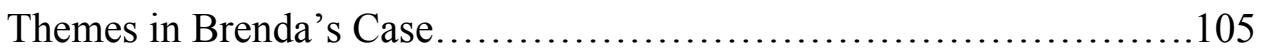

Case 2: Linda..................................................... 106

Themes in Linda's Case...........................................111

Case 3: Monica.................................................... 112

Themes in Monica's Case...........................................119

Case 4: Mary ..................................................... 120

Themes in Mary's Case.........................................124

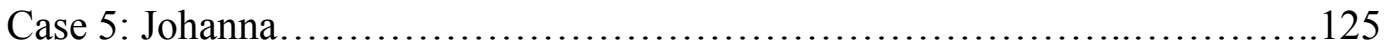

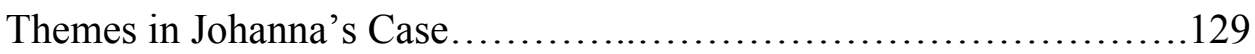

Cross-Case Themes.................................................... 131

Culturally Responsive Implementation of Scripted Curricula...........132

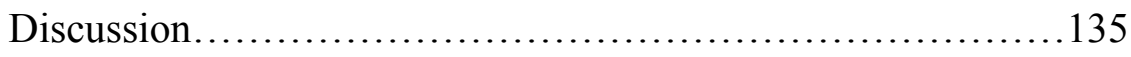

Relationships as a Key to Better Understanding Focal Students.........138 
Discussion.....................................................140

Teacher Collaboration.............................................. 142

Discussion .................................................144

Home Visits.................................................. 145

Discussion....................................................148

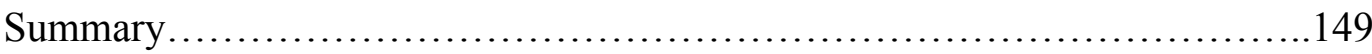

V Discussion and Conclusions............................................. 150

Summary of the Study ................................................... 150

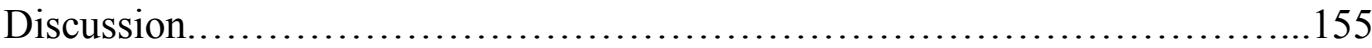

Fidelity vs. Integrity............................................... 155

Potential Limitations for Culturally Responsive Scripted Instruction...................................................... 157

Below the Line Information vs. Funds of Knowledge...................161

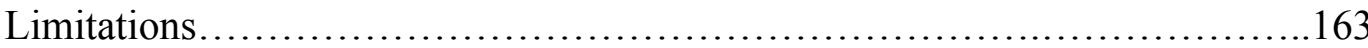

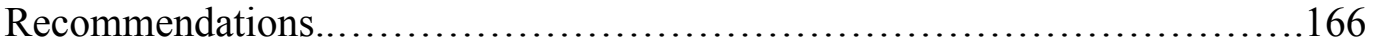

Future Research...................................................166

In-Service Teacher Professional Development........................168

Culturally Responsive Teaching Combined with Scripted Curricula.....170

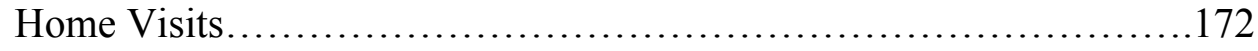

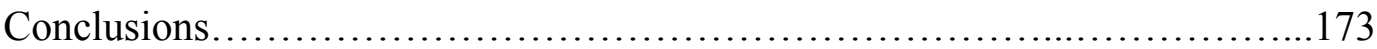

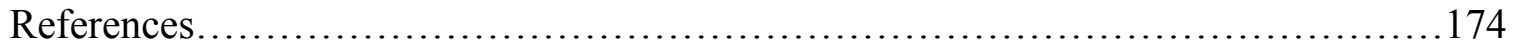


Appendices

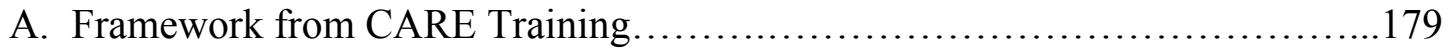

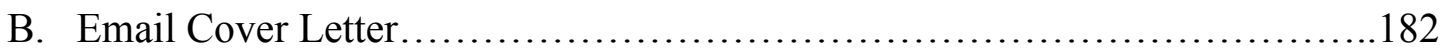

C. Letter of Informed Consent............................................... 183

D. Email Reminder......................................................... 185

E. Questionnaire to Determine Maximum Variation

Among Sample Population................................................. 186

F. Protocol for Individual Interviews...................................... 187

G. Protocol for Group Conversation.......................................189

H. Data Collection Timeline................................................. 190 


\section{LIST OF TABLES}

Table

Page

1 Components of Culturally Responsive Instruction Based on Gay (2010)...........9

2 Foundational Curriculum Theories Based on Kliebard (2004)....................25

3 Courageous Conversations Agreements and Conditions from Singleton

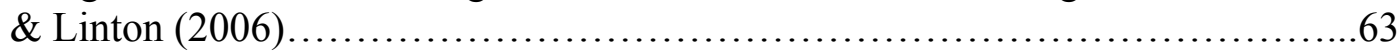

4 Framework for Culturally Relevant Teaching................................65

5 Overview of Coding Strategies and Their Purpose...............................84

6 Frequencies of Most Repeated Codes......................................92

7 Master List of Reoccurring Codes.........................................93

8 Summary of Within-Case and Cross-Case Themes...........................96

9 District-Adopted Curricula Used in CARE Lessons...........................97

10 Summary of the Relationship Between Cross-Case Themes and Assertions.........153

11 Summary of Answers to the Study's Research Questions..........................154

12 How This Study's Findings Represent the Components of Culturally Responsive Instruction and Multicultural Education as Described By Gay and Banks.........159 


\section{LIST OF FIGURES}

$\begin{array}{lll}\text { Figure } & \text { Page }\end{array}$

1 In-Depth Portrait of Case Study Data Analysis (Creswell, 2007)..................82

2 Phases of Data Analysis................................................... 83

3 Visual Organization of Research Questions and Codes............................88

4 Relationship Between Funds of Knowledge and Below the Line Information......162 


\section{CHAPTER I}

\section{INTRODUCTION}

The passing of No Child Left Behind (NCLB) in 2002 has led to many significant changes to schools nationwide (“Understanding NCLB,” 2007) particularly with respect to reading instruction. The far-reaching goals NCLB imparts, as well as the mandates set forth as means to reach those goals, have focused much attention on reading instruction and put pressure on teachers to help students improve their reading skills. NCLB ambitiously set out to ensure that all students would be able read at grade level no later than the end of third grade. Additionally it aims to identify and close the achievement gap that exists between culturally and linguistically diverse students and their White peers, to ensure that each state makes adequate yearly progress (AYP), and to ensure that all students will be proficient in reading by the end of the 2013-2014 school year ("Understanding NCLB," 2007). As stated in the law, these goals are monitored by annually testing students using the same academic assessments to measure achievement. NCLB requires implementation of reading instruction that is referred to as scientifically research-based in order to propel students towards achieving the intended outcomes the law sets forth. NCLB defines scientifically-based research as research that:

Applies rigorous, systematic and objective procedures to obtain valid knowledge relevant to reading development, reading instruction, and reading difficulties, and includes research that employs systematic, empirical methods that draw on observation or experiment, involves rigorous data analyses that are adequate to test the stated hypothesis and justify the general conclusions drawn; [and] relies on measurements or observational methods that provide valid data across evaluators and observers and across multiple measurements and observations (Title 1, Part B, Subpart 1, Section 1208). 
The increased focus on the implementation of scientifically research-based instruction that addresses phonics, phonemic awareness, vocabulary development, comprehension, and fluency in oral reading, as well as the use of standardized assessments to measure and track student progress in these areas ("Understanding NCLB," 2007), has resulted in the widespread use of scripted core reading programs as the primary vehicle for classroom reading instruction (Dewitz, Leahy, Jones, and Sullivan, 2010). Dewitz et al. describe scripted core reading programs as, "the central, essential part of every reading program — no other texts needed" (p.32), a statement which lends itself to the assumption that this type of program includes the "right" materials and steps to help students accomplish the goals set forth by NCLB and, therefore, would not require teachers to supplement with additional activities or materials based on their knowledge of their particular students.

However, nearly a decade after the inception of NCLB, the achievement gap persists (Howard, 2010). Despite efforts to standardize and streamline reading instruction ("Understanding NCLB," 2007) the "discrepancy in educational outcomes between various student groups, namely African American, Native American, certain Asian American, and Latino students on the low end of the performance scale, and primarily White and other Asian American students at the higher end of the performance scale" (Howard, p.10) has not been remedied. The differences in achievement between White students and most students who are culturally and linguistically diverse is an indicator that certain students, most often White and economically advantaged students, have the prerequisite background knowledge that is relevant for making sense of the scripted 
content and included texts (Hirsch, 2007). I believe, however, that particular students having background knowledge that is relevant to the curricular content does not mean that their perspective is superior or that their lived experiences count more than those of culturally and linguistically diverse students.

To choose what should be taught in schools inevitably results in the validation of certain kinds of knowledge or skills as more valuable to students, and therefore more worthy of being part of formal schooling, than the knowledge and skills that are left out. In an era where the number of culturally and linguistically diverse students is increasing rapidly (Ginsberg, 2007; Schmidt \& Lazar, 2011) while concurrently standardization, scripted programs, teaching to the test, and data driven practices are the norms in classrooms (Ravitch, 2010; Sleeter, 2005), it is essential to consider that educational practices relying entirely on prefabricated content may benefit certain students more than others. As a professional, I am calling for recognition that all students bring a wealth of knowledge to the classroom, which should be acknowledged, respected, valued and incorporated into instruction (Gonzalez, Moll, \& Amanti, 2005; Schmidt \& Lazar, 2011; Vélez-Ibañez \& Greenberg, 1992). My argument stands on the concept of funds of knowledge (Veléz-Ibañez, 1988) as referenced by Moll, Amanti, Neff, \& Gonzalez (1992) and Gonzalez et al., which is based on the premise that all people are competent, all people have knowledge, and people's life experiences give them that knowledge (Boske \& Benavente-McEnery, 2010).

I believe that in order to promote better learning experiences for culturally and linguistically diverse students, teachers need the flexibility to enhance scripted curricula 
to more explicitly foster connections between the content and students' background knowledge. Initially, I intended to frame my research entirely around the argument favoring a modification of scripted reading programs, yet as I explored the topic further, I realized that there is a deeper and more fundamental level of the problem. Even if teachers are granted the time and permission to modify scripted content in order to build bridges between the scripted content and students lived experiences, doing so is not easily accomplished when the lives of educators are disconnected from their students (Baeder, 2010). The differences between the respective lived experiences of teachers and their students left me wondering how teachers can familiarize themselves with students' funds of knowledge in order to go about providing culturally and linguistically diverse students with more equitable learning experiences. With this in mind, I decided to investigate how teachers become familiar with students' funds of knowledge in addition to how teachers can utilize students' funds of knowledge as an essential component of culturally responsive instruction in order to enhance scripted curricula to create improved learning opportunities for culturally and linguistically diverse students. In the following section, I address issues of inequality for culturally and linguistically different students. Additionally, I will clarify what culturally responsive lessons and the utilization of students' funds of knowledge can contribute to instruction and the implications for a more equitable learning environment for culturally and linguistically diverse students.

\section{Inequalities for Culturally and Linguistically Diverse Students}

Howard (2010) describes getting an education, by means of public schooling, as the proverbial equalizer which functions as, "the commodity that helps to transform life 
chances, improve economic prospects, change dire outlooks to promising possibilities, and reduce the gap between the haves and the have-nots" (p.9). This trust in the power of education is a common belief held by our society that champions education as a life changing experience (Nieto \& Bode, 2008). Yet, Howard points out that previous exposure to certain information and particular kinds of experiences, which Delpit (2012) identifies as middle-class home culture, privileges some students. Delpit indicates that consequently, it is assumed there is something wrong with students who do not exhibit knowledge of middle-class home culture and that they lack basic intelligence. Howard references Banks (1999) to explain that middle class, White culture is embedded in the concepts, paradigms, and experiences that are presented as mainstream academic knowledge, posing a problem for culturally and linguistically diverse students whose personal experiences at home and with their families may produce explanations, interpretations, and values that differ from those associated with academic proficiency within the school setting.

The concerns I have over the prevalent use of scripted core reading programs emphasizing the instruction of predetermined information that is taught in prescribed ways (Dewitz et al., 2010) is nested within a larger educational issue of inequity in educational experiences between students who are White and students who are not. I believe educational experiences are inequitable because of the extent to which scripted programs normalize Eurocentric and middle class forms of discourse, knowledge, language, culture, and historical interpretations as academic knowledge (Howard, 2010) without providing space for teachers to include culturally relevant material and/or 
practices. Given this, it is difficult to view education as the proverbial equalizer, when there are clear distinctions between how various students can access the benefits of education which corresponds to the extent to which their personal discourse, knowledge, language, and culture are reflected in the scripted content. This critique is not meant to discredit the effectiveness of scripted core reading programs or to attack the protocol of teaching such programs with fidelity. In fact, I use a scripted phonics program with my Kindergarten students and it is very effective. Rather, my argument is that educators have the potential to greatly enhance the impact of scripted content by drawing upon the life experiences of students in order to foster connections between the content being taught and the experiences students have outside of school. The incorporation of multiple perspectives and students' lived experiences provides an excellent context for promoting understanding, acceptance, and appreciation for differences that are inherent within diverse student populations.

Shannon (1998) notes that one of the traditional rationales for schooling is to "encourage students to broaden their local perspectives - to expand their horizons beyond their communities—in order that schools prepare them for life" (p.5); yet, this does not occur when there is one predominant perspective that is represented in scripted content. According to this outlook, public schooling would ideally function to expose students to different cultural norms in a positive way in order to remove cultural barriers (Shannon). However, Shannon points out that cultural differences are treated as natural deficits and that school norms are in fact, "biased social constructions based on social class, race, and language in order to preserve the privileges enjoyed by the 'normal' (that is, 
economically advantaged, white, and standard English speaking) youth” (p.5). VelézIbañez and Greenberg (1992) concur that public schools rely on a deficiency model that under utilizes the funds of knowledge contained in the households of culturally and linguistically diverse students. Consequently, societal stratifications are kept intact and culturally and linguistically diverse students continue to achieve at lower levels than their White peers (Howard, 2010).

Delpit (2012) indicates that when students don't display knowledge of basic skills, which she describes as, "knowledge of the strategies and conventions of middleclass cultural capital" (p.55) it is the students and their families that are seen as deficient rather than the instruction. Additionally, the perspective that "white" is good and superior is so ingrained and normalized throughout our society that, "we miss the pain in our children's eyes when they have internalized the societal belief that they are dumb, unmotivated, and dispensable" (Delpit, Introduction, paragraph 14). There is so much focus on what these students don't know rather than an exploration of what they do know. Delpit calls for recognizing the brilliance of culturally and linguistically diverse students lest we continue to perpetuate, "stereotypic societal views that these children are somehow damaged goods and that they cannot be expected to succeed" ("There is No Achievement Gap,” paragraph 8).

\section{Culturally Responsive Instruction}

In order to interrupt the current trend where stratifications among student achievement are perpetuated (Nieto \& Bode, 2008; Grant \& Sleeter, 2003), I argue reading instruction needs to help students experience reading and writing in ways that 
draw from and apply to their lives, families, cultural identifications, and future aspirations allowing them to make sense of new information in terms of what they already know. I gravitate towards Delpit's (2012) description of the need for a "consciously devised, continuous program that teaches skills and develops vocabulary in the context of real experiences, provides rigorous instruction, connects new information to the cultural frameworks that children bring to school, and assumes that the children are brilliant and capable" ("Learning at Home and at School," paragraph 9). In order to realize the type of instruction Delpit speaks of, I suggest that culturally responsive literacy instruction should be incorporated into the use of scripted core reading programs as a means to foster deeper understanding and contextualization of reading instruction as well as introduce multiple perspectives with regards to literacy so that White, middleclass values are not seen as the 'right' way or the only way to interpret the content being taught.

Culturally responsive instruction uses, "cultural knowledge, prior experiences, frames of reference, and performance styles of ethnically diverse students to make learning encounters more relevant to and effective for them" (Gay, 2010, p.31), while at the same time ensuring that students are exposed to different ways of thinking based on the multiple perspectives included in instruction. My support for culturally responsive instruction stems from the literature and research from scholars such as Gay (2002; 2010), Banks (2001), Ladson-Billings (1992a; 1992b; 1995, 1999), Moll et al. (1992) and Veléz-Ibañez \& Greenberg (1992) who align themselves with teaching practices incorporating the background experiences of students and emphasize making instruction 
relevant to students' frames of reference in order to accelerate student achievement and deepen understanding. Table 1 summarizes the components of curriculum and instruction for culturally and linguistically diverse students as identified by Gay (2010).

Table 1

Components of Culturally Responsive Instruction Based on Gay (2010)

\begin{tabular}{ll}
\hline Component & Brief Description \\
\hline Culturally Responsive Caring & $\begin{array}{l}\text { Caring for instead of about } \\
\text { students }\end{array}$ \\
Culture and Communication in the & $\begin{array}{l}\text { Understanding and } \\
\text { accepting students' cultural } \\
\text { classroom }\end{array}$ \\
Cultural Congruity in Teaching and & $\begin{array}{l}\text { Conveying knowledge } \\
\text { through students' learning }\end{array}$ \\
Learning & styles \\
& Making content accessible \\
Ethnic and Culturally Diverse & by connecting it to students \\
Curriculum Content & lives outside of school \\
\hline
\end{tabular}

I have chosen to focus on the components of culturally responsive instruction as described by Gay to provide a more in depth description of each component.

Gay (2010) refers to the act of culturally responsive caring as, "caring for instead of about the personal well-being and academic success of ethnically diverse students" (p.48) with the distinction that caring for students is an active engagement in doing something to positively affect students' well being rather than simply feeling concern. Culturally responsive caring manifests itself, "in the form of teacher attitudes, expectations, and behaviors about students' human value, intellectual capacity, and performance responsibilities" (p.48). Gay notes that caring teachers place students at the center of learning by using their interests and strengths into opportunities for academic 
success.

Communication is a key component in culturally responsive instruction because, as Gay (2010) describes, there is a "semiotic relationship among communication, culture, teaching and learning" (p.76). Gay calls teachers to understand and accept students' cultural communication styles in order to avoid communicative mismatches that may hinder academic performance. Gay references Boggs (1985) who held that, "the form of exchange between child and adult and the conditions in which it occurs will affect not only what is said, but how involved the child will become" (p.79).

Cultural congruity in teaching and learning reflects the need for teachers to understand how ethnically diverse students learn because, "the process of learning — not the intellectual capacity to do so-used by students from different ethnic groups are influenced by their cultural socialization" (Gay, 2010, p. 174). Teachers can help students achieve success by conveying knowledge through students' learning styles which should take into account procedural, communicative, substantive, environmental, organizational, and perceptual, relational, and motivational dimensions of learning.

Finally, ethnic and cultural diversity in curriculum content aims to empower ethnically diverse students by making content knowledge accessible to students by connecting it to their lives and experiences outside of school. It is this component of culturally responsive instruction that best relates to the concept of funds of knowledge on which this study is framed. Curricular content should include "histories, heritages, contributions, perspectives, and experiences of different ethnic groups and individuals, taught in diverse ways" (Gay, 2010, p.127). 
Culturally responsive caring, cultural communication, cultural congruity in teaching and learning as well as ethnic and cultural diversity in curriculum content all contribute to more effective educational experiences for culturally and linguistically diverse students. Because each of these components aims to specialize instruction for children that represent various ethnic and cultural groups, it is imperative to examine the potential for achieving these components while implementing scripted curricula.

Many scripted programs include separate instruction manuals or specific teaching strategies for use with English Language learners because, as noted by Goldenberg (2008), modifying instruction should into account students' language levels as a key aspect of improving education for children from non-English speaking homes. Students' native languages are a significant aspect of their culture; yet, I think that only focusing on language in terms of helping students acquire the skills in English they "lack" rather than fostering connections between native languages and English or utilizing native languages to enhance understanding or communication, does not achieve culturally responsive communication as described by Gay (2010). I contend that making adjustments to the language used in instruction needs to be paired with Gay's other components in order to provide additional pathways to support the achievement and engagement of culturally and linguistically diverse students.

Gay's perspective on culturally responsive instruction helps to support my claim that there is an inherent problem in the widespread usage of scripted core programs if they are used as the only resource for reading instruction because of the extent to which they do not extensively integrate students' frames of reference (Sleeter, 2005). Gay 
affirms the significance of matching curriculum more closely to the particular students in a particular classroom in order to utilize their own experiences as a foundation for learning, which is less likely to take place if teachers only use curricula that were created outside the context of what their particular students know and how they experience the world.

Sleeter (2005) also supports this claim with the indication that the use of scripted curricula reduces the likelihood that curriculum will be culturally relevant to one's own students and that it extensively fractures the curriculum, eliminating opportunities for teachers to utilize their own judgment about what to teach, which would be based on what they know about their students. Sleeter suggests that students are subjected to "one size fits all" standardized curricula that often perpetuate stereotypes and racism, which is indicative of curricula not reflective of the principles of multicultural education. I do not have the same degree of opposition to the use of scripted curricula as Sleeter; yet, I agree that scripted programs should not be used as a replacement for teachers' expertise about their craft and their knowledge of their students. When scripted curricula are used in the absence of teachers' reflections on how the scripted content might be perceived by culturally and linguistically diverse students or mismatched to their experiences, these students do not have the same access to learning opportunities that draw upon their specific background knowledge and personal schemas if the content in the scripted lessons does not resonate with what is familiar to them (Howard, 2010). Again, I would like to reiterate that I am not favoring the elimination of scripted core reading programs in exchange for a more constructivist approach to reading instruction; rather, I support the 
need for teachers to be active participants in evaluating scripted instruction and enhancing the prefabricated content based on their understanding of and familiarity with students' funds of knowledge rather than simply relying entirely on a prefabricated script.

\section{Utilizing Students' Funds of Knowledge}

Literature that focuses on funds of knowledge helps to clarify the significance of being familiar with, and drawing from, students' experiences at home to enhance curriculum. Informed by the work of Veléz-Ibañez (1988), Moll et al. (1992) refer to "funds of knowledge" as "historically accumulated and culturally developed bodies of knowledge and skills essential for household or individual functioning and well-being" (p. 133). In terms of learning, funds of knowledge refer to the experiences, frames of reference, and perspectives students gain from their households, which they subsequently bring to the learning environment at school. Students use these funds of knowledge as a base on which they build in the process of learning. Utilizing students' funds of knowledge in order to facilitate better connections to new information is a core component of culturally responsive instruction, and aligns with Banks' (2001) content integration and equity pedagogy dimensions as well as Ladson-Billings' (1995) cultural competence tenet of culturally responsive instruction. Even small bits of knowledge such as the names of students' siblings, family size, where students live and/or parents' occupations can positively influence how connected culturally and linguistically diverse students feel if teachers' incorporate that knowledge into instruction. Drawing upon students' funds of knowledge requires going beyond the script in order to craft instruction that reflects familiarity with students and families as opposed to teaching the same exact 
content year to year regardless of changes in student demographics, cultural affiliations, and/or students' home languages.

The idea of teachers' enhancing scripted content by incorporating students' funds of knowledge seems logical; yet, it poses a problem for teachers if they are not familiar with the lives students lead outside of school and if the teachers' lives are quite different from those of their students. My curiosity regarding how teachers get to know their students at a deeper level brought me back to my attitudes when I first became a teacher and fuels my interest in searching for literature that explains and clarifies how teachers gain access to students' funds of knowledge.

\section{My Interests}

In my teacher preparation program, I learned how to teach middle class white students, which is reflective of my own background, and that is exactly what I set out to do when I began looking for my first teaching job after moving to Portland. In learning about pedagogy and instructional strategies, there was no attention in my coursework to working with children who were acquiring English or who were not White. Having grown up in a monolingual, White environment, I had no awareness that what I was learning was lacking a certain depth that would merit it applicable to our increasingly diverse society. I began researching school districts in the Portland area that I wanted to apply to and I can recall, having a different perspective on diversity than I do now, paying particular attention to the demographics of the schools. Portland Public School District has many elementary schools and I was most interested in the schools that had a large percentage of White students. I felt more comfortable not applying to the schools 
that had large populations of African American or Hispanic students and made my decisions about where to apply based on the percentages of White students attending the schools, consequently crossing the diverse schools off my list. I wanted to teach people who were like me because that is what I knew how to do. Looking back, and reflecting on the limited view of what kind of job I "wanted," I think I am fortunate for how things worked out.

After not being able to get a job teaching at the elementary level, I took a position as a preschool teacher in a bilingual Head Start classroom. One of my requirements as a Head Start teacher was to conduct visits to my students' homes. The experiences of seeing students in their home environment, how they interacted with their parents, what their living space looked like, and how the families treated me as a guest in their house, contributed very much to my current interest in students' funds of knowledge and the role they play as a contributing factor for classroom and school success. Visiting homes wasn't always comfortable or easy; yet, it provided me with a new perspective on my students and their families. Nine months as a Head Start teacher had a profound impact on the teacher I have become. Time spent working with migrant children and their families as well as teaching in a classroom where all of the students were Latino and native Spanish speakers was time invested in changing my beliefs about my purpose in teaching. I no longer wanted to avoid the schools that housed the brown and black students; rather those students became the ones whom I desired more than anything to work with. I was tired of being naïve to differences and wanted to dive into alternative experiences where I could learn more about people who were quite different from me. At 
this point, despite the changes in my awareness about teaching non-White children, my attitude was still very much centered on myself and what I was learning, rather than truly recognizing the need to rethink myself and my teaching practices given the students in my classroom.

Once I secured a job teaching first grade in a public school, I continued teaching many students from Spanish speaking backgrounds. I felt very confident about working with these students and families because of my previous experience in Head Start. I felt very equipped with many strategies to help them acquire English because at that point because I had returned to graduate school to get an ESOL endorsement. In my first two years of teaching I completed all the courses in the endorsement program and learned a lot about language acquisition. I conducted my own parent teacher conferences, in Spanish, rather than getting a translator, and I truly enjoyed working with these native Spanish speakers. It felt good to be a teacher in a classroom where the sea of faces looking back at me were not all white like the ones in my own elementary classrooms. It felt empowering to know that I could communicate with these families when other teachers could not. I felt like I was going to make a difference, be a different kind of teacher, and that I would be successful in teaching my students. I had a sense of satisfaction because I was adding some color to my very monochromatic life. At the same time, when handed incomplete, incorrect, or insufficient work from one of these Latino students, my inner dialogue said, it's okay, they just don't know better. I didn't realize it at the time, in the midst of my inflated sense of self-importance, but I still had a lot of learning to do. I loved all my students equally, that is for sure; yet, I lowered my 
expectations for certain students based on their language abilities, which were grounded in their cultural identities.

In my third year of teaching, I began participation in a workshop series called Courageous Conversations, which I have continued to be a part of since. Courageous Conversations is an anti-racism awareness program that addresses the many issues surrounding institutionalized racism in our educational system. The process of this training has been most influential on my own personal awareness of myself as an educator thus far in my career. Throughout the course of this program, I learned a lot about "Whiteness" and how much being White plays a part throughout the course of one's life. Because of my race, I am part of the majority culture in the United States and I belong to a culture of privilege. Many of my students do not have access to this privilege, simply based on the color of their skin.

In subsequent years, I joined other branches of this training and became part of the Partnerships for Academically Successful Students (PASS) and Collaborative Action Research for Equity (CARE) teams at my school. Each school year I have participated in additional series of trainings focused on better meeting the educational needs of culturally and linguistically diverse students with emphases on working with parents of color (PASS) and culturally responsive instruction (CARE). All of the work related to Courageous Conversations has helped me engage in active reflection on the practices I use in the classroom and how culturally and linguistically diverse students experience education in our country, in the school district I work for, and more particularly in my classroom. As a result, I have learned to think about multiple perspectives and consider 
how educational practices that normalize White, middle class values can stunt the academic success and learning opportunities for non-White students. Therefore, I feel committed to remaining open-minded in my pedagogy so that I can foster the appreciation of differences among my students and maintain high expectations for all students, while at the same time nurturing the need all students have to feel a sense of belonging in the classroom and connection to school content. It is with these beliefs in mind that I commit myself to ensuring that my instructional practices are grounded in the literature on best practices for culturally and linguistically diverse students representing deep reflection on better meeting the needs of students whose values, beliefs, and funds of knowledge are not as readily present within scripted instruction.

It is with this work in mind that I have taken interest particularly in reading instruction for culturally and linguistically diverse students and how students' funds of knowledge can be woven into the use of scripted core reading programs. My interest and inquiry into this area does not come with a simple answer, therefore, as I previously mentioned, the main focus for this research approach is how teachers like me can become familiar enough with students' funds of knowledge to be prepared for using this information effectively as a resource for classroom instruction in conjunction with the use of scripted curricula. Although my main focus is on enhancing scripted core reading programs with culturally responsive instructional strategies, I recognize that it is also significant to explore how teachers modify scripted curricula for science, math, and other subject areas and how students' funds of knowledge can be applied to those subjects areas as well. 


\section{Role of Researcher}

As a classroom teacher, I am faced with the challenge of instructing many culturally and linguistically diverse students who do not share my own background and life experiences. My school district has placed a great emphasis on reducing the racialized achievement gap and better meeting the needs of culturally and linguistically diverse students; yet, culturally responsive instruction is a complicated pedagogy without a clear path for proper implementation. As an employee of a district that has adopted a scripted reading program as well as scripted programs in other content areas, I often struggle to find a balance between teaching the program with fidelity as it was intended and trying to make my instruction more culturally relevant by enhancing or altering the scripted content in an effort to make it more compelling, appropriate, or relevant for culturally and linguistically diverse students.

As a professional committed to being a culturally relevant educator who continuously examines my practice and reflects on how my instruction is or is not meeting the needs of culturally and linguistically diverse students, I am continuously looking for ways to make the scripted content more accessible for this population of students. Although I am dedicated to trying new strategies and including practices that are more culturally responsive, I find it difficult to know exactly what actions and/or strategies will actually be more culturally relevant for the students in my classes. My review of literature in the area of funds of knowledge has peaked my interest in the practice of home visits as a way for teachers to better understand culturally and linguistically diverse students and their families; yet, I recognize that home visits are not 
the only way for teachers to access information about their students and the students' families that would contribute to their abilities to make lessons more culturally responsive. A better understanding of what life is like for these students and families outside of the classroom and the school can aide teachers in making pedagogical decisions in the best interest of these children.

As the researcher, I am interested in better understanding how other teachers in District $\mathrm{CC}$ who implement scripted curricula have experienced the process of creating culturally responsive lessons and becoming more familiar with students in their classes who are culturally and linguistically diverse. However, at the same time, conversations with other teachers and the content that will be covered in this investigation will undoubtedly impact my own personal understanding of the process and will likely impact my own attitudes and perceptions about the process. I will work hard to ensure that my own perceptions are not projected onto participants and to focus on hearing what participants are sharing about their experiences in order to uncover themes and contribute some new ideas to body of literature that exists.

\section{Statement of the Purpose}

The goals of this study were to explore how teachers in District CC, who utilize district-adopted scripted curricula, adapt the prescribed curriculum for culturally and ethnically diverse students as well as to investigate what behaviors and/or ideas teachers have for becoming familiar with students' funds of knowledge. The study was designed with the purpose of contributing to the knowledge base regarding how the seemingly separate bodies of literature pertaining to both implementation of scripted curricula and 
the creation of culturally responsive instruction can be combined to elicit more equitable and effective instruction for culturally and linguistically diverse students.

Qualitative methods were used to capture teachers' perceptions about the culturally responsive lessons they planned to engage their culturally and linguistically diverse students and how those lessons impacted their focal students. Qualitative measures were used to identify themes pertaining to how teachers learned more about their culturally and linguistically diverse students as well as ways in which they used district-adopted scripted curricula while implementing culturally responsive lessons. Results were intended to inform the field of the potential for culturally responsive instruction to be used in conjunction with scripted curricula as well as to inform practice by providing teachers with detailed descriptions of what lessons can look like when they are based on the implementation of prescribed curricula, but also focus on utilizing culturally responsive instructional practices.

\section{Research Questions}

Based on the purpose of this study, the overarching research questions were:

- R.Q.1 How do teachers who implement scripted curricula describe the experience of creating culturally responsive lessons intended to specifically connect with their culturally and linguistically diverse students' funds of knowledge?

- What do teachers report about the ways in which they draw from or incorporate students' funds of knowledge in their instruction?

- How do teachers perceive the impact of culturally responsive instruction for culturally and linguistically diverse students in their classrooms? 
- R.Q.2 What do teachers report regarding the ideas and behaviors they have developed as ways to better understand their culturally and linguistically diverse students and their families?

- What do teachers report about factors that influence their willingness to conduct home visits as an avenue to better understand culturally and linguistically diverse students and their families?

In the following chapter I will discuss four foundational curriculum theories that shed light on the attitudes and values that support different purposes for the development of curriculum. The differences in conceptualizations of what curricula should entail are reflected in variations of contemporary educational practices. After briefly outlining four influential theories that played a significant role in shaping how curriculum is conceptualized, as well as John Dewey's ideas about the extent to which the curriculum should relate to the child, I will discuss what reading instruction looks like today under the mandates set forth by NCLB. This research effort also calls attention to the learning needs and outcomes for culturally and linguistically diverse students through reviews of literature in the areas of culturally responsive pedagogy and funds of knowledge. Each of these sections aims to describe educational practices that benefit culturally and linguistically diverse students that are not always used in conjunction with scripted curricula. 


\section{CHAPTER II}

\section{REVIEW OF THE LITERATURE}

This chapter reviews literature in the areas of curriculum theory, reading instruction in the era of No Child Left Behind, culturally responsive instruction, and funds of knowledge in order to contextualize the foundational literature on which this study was based. Additionally, I will address the gap in the literature that inspired the research questions in the study.

\section{Foundations of Curriculum Theory}

When considering the state of education in the context of our pluralistic, technological, globally economical, and continuously growing society, returning to the theoretical frameworks of curriculum helps to contextualize educational values currently prevailing and the depths of their roots. The same disagreements over what should be taught that take place today were also the source of much educational debate as early as the $19^{\text {th }}$ century and the curricular theories that arose then still play a significant role in

contemporary education. The theories I discuss emerged in the early $19^{\text {th }}$ century, creating contention among scholars regarding the function of curriculum and what factors should be addressed in curriculum as a means of preparing students for future endeavors in their lives. In this section, I describe four influential curriculum theories and present a table outlining the main beliefs and ideas associated with each. I have also chosen to include John Dewey's ideas about the child and the curriculum as his work is integral in the field of curriculum development. 


\section{Historical Background of Curriculum Debates}

As America transformed into an urban, industrial nation throughout the $19^{\text {th }}$ century, the growth of railroads, increased newspaper readership, and population increases aroused greater public awareness of the social changes the country was undergoing as well as uncertainty regarding the country's future (Kliebard, 2004). Kliebard notes that this increased social awareness led to a new vision of the significance of schooling in shaping American society. This new interpretation of the role of schooling consequently influenced a change in the educational center of gravity, which led to increased attention to the nature of curriculum and the desire for change within the traditional education.

Until the end of the $19^{\text {th }}$ century, the mental disciplinarian doctrine influenced curriculum, seeking to strengthen the powers of the mind (develop the ability to think) and fill the mind with content (learn knowledge and skills) as described by Yale faculty members Jeremiah Day and James Kingsley who supported the mind-as-a-muscle metaphor in their 1828 report (Kliebard, 2004). The influences of this metaphor for learning were evident in the monotonous drill, hard discipline, and mindless verbatim recitation that were prominent aspects of the school regime in order to strengthen the brain. Preservation of the curricular status quo was desirable for some; however, the increased focus on the course of study in U.S. schools as a result of the rapidly changing society triggered decades of contention between educational scholars over what curriculum should entail, what students should learn in school in order to meet the 
demands of the new industrial society, and how the curriculum needed to adapt to prepare students for their future endeavors.

Further Kliebard (2004) notes that during the $20^{\text {th }}$ century four distinct interest groups of educational scholars-the humanists, developmentalists, social efficiency educators, and social meliorists-held ground in the continued debate over curricular supremacy. Each group valued and supported different versions of what knowledge is worth teaching and the desired functions of schooling; they competed for dominance in order to shape the nature American curriculum according to their beliefs. Table 2 provides brief descriptions of the ideas each group represented in order to clarify the distinct perspectives present in the academic arena of school reform beginning in the late $19^{\text {th }}$ century.

Table 2

Foundational Curriculum Theories Based on Kliebard (2004)

\begin{tabular}{ll}
\hline Curriculum Theory & Basic Beliefs \\
\hline Humanism & $\begin{array}{l}\text { Sought to preserve traditions and values of Western civilization } \\
\text { and to continue emphasis on development of reason in the fac } \\
\text { of rapid change and burgeoning school system }\end{array}$ \\
Bevelopmentalism & $\begin{array}{l}\text { Believed that curriculum should be informed by scientific data } \\
\text { with respect to stages of child development and the nature of } \\
\text { learning in order to harmonize with the child's real interests, } \\
\text { needs, and learning patterns }\end{array}$ \\
Social Efficiency & $\begin{array}{l}\text { Prioritized creating an efficient, smoothly running society by } \\
\text { applying standardized techniques of industry to the business of } \\
\text { schooling }\end{array}$ \\
Social Meliorism & $\begin{array}{l}\text { Saw schools as a principle force for social change and social } \\
\text { justice with the power to create a new, more equitable social } \\
\text { vision }\end{array}$
\end{tabular}

Sought to preserve traditions and values of Western civilization and to continue emphasis on development of reason in the face with respect to stages of child development and the nature of learning in order to harmonize with the child's real interests, needs, and learning patterns

Prioritized creating an efficient, smoothly running society by applying standardized techniques of industry to the business of schooling justice with the power to create a new, more equitable social vision 
The four factions of curriculum theory outlined in Table 2 are significant because they continue to influence contemporary pedagogy. More than a hundred years later, scholars and educators still debate best practices, what curricula should entail, and the ultimate mission of schooling for students in the United States. For example, modern scholars who emphasize the need for multicultural education and culturally responsive instruction are still calling for major reform movements in education as they strive for an educational system that better suits the needs of culturally and linguistically diverse students as well as students living in poverty within our pluralistic society (Banks, 1999; Banks, 2001; Gay 2010; Howard, 2010). Later in this section, I will discuss how the quest for equity in schools and the vision of social justice as promoted by the Social Meliorists is very limited in the context of the scripted curricula that go hand in hand with NCLB.

\section{John Dewey-The Child in the Curriculum}

In addition to the aforementioned groups, John Dewey's work also greatly influenced beliefs about curriculum and instructional practices. I believe Dewey's perspective about the importance of acknowledging the child in the curriculum relates to my concern for culturally and linguistically diverse students whose background knowledge and lived experiences, if utilized for instructional purposes in the classroom, have the potential to positively impact their educational experiences. Therefore, I have chosen to include his theories about curriculum, which transform the ideas of the previously discussed theories in Dewey's own distinct theory. 
Dewey's (1902) perspective on curriculum touches upon the tenets of other previously mentioned curricular theories; yet, Dewey's outlook is indeed unique and therefore worthy of mention. His point of view represents a delicate balance between the "old education" that ignores the dynamic nature of the individual child in order to propel him on a controlled path and the "new education" that provides the child with ample room to discover and figure things out on his own without sufficient guidance. Dewey proclaims that, "a map does not take the place of an actual journey" (p. 26) meaning the experience of the child cannot be disregarded in the process of teaching content. Yet, he acknowledges that the map still has an essential function, which is to guide, provide direction, and point out paths that quickly lead to a desired result. Dewey calls for a combination of delivering the content knowledge to passive students, which brings to mind Friere's (2007) notion of the banking effect, while also connecting to students by bridging content with the life experiences they bring to the classroom. Dewey cautions that prioritizing the content or subject matter to be taught de-emphasizes the role of the learner; however, on the other hand, excessive focus on the child can diminish the role of specific content and the role of the teacher as well. Dewey's perspective is similar to my own in that, I believe a scripted program provides a very well planned and essential roadmap for curriculum and instruction; yet, the script cannot be used as a map without being informed by the teacher's knowledge of and connection with his or her students. Similar to Gay (2010) and Moll et al. (1992) who advocate for culturally responsive instruction, Dewey asserts that an essential component of education requires that, "School must represent present life—-life as real and vital to the child as that which 
he carries on at home, in the neighborhood, or on the playground" (Dewey, 1987, p.7). The child's personal experiences should be the true center of correlation for different subjects within the curriculum as the child engages in a continuing reconstruction of experience while making sense of new information by means of incorporation with prior knowledge. Dewey notes several problems that arise when the subject matter has no direct relationship to the experiences of the child. He indicates that without any organic connection to what the child has seen or experienced, the content is simply formal and symbolic rather than meaningful. Additionally, he notes that content presented in the most logical fashion, which he describes as, "a lesson to be learned as a lesson" (p. 33), results in decreased motivation from the child as needs to undergo some modification in order to truly resonate and take on meaning.

Dewey's (1902) emphasis on the importance of recognizing the child within the curriculum to more effectively position the curriculum in accordance with how the child sees and understands the world shares some ground with the theory of developmentalism. The developmentalists assumed that, "the natural order of development in the child was the most significant and scientifically defensible basis for determining what should be taught" (Kliebard, 2004, p. 11). Both Dewey and the developmentalists emphasized the importance of aligning curriculum with the interests, needs, and learning patterns of the child; however, Dewey considered his views to be a reinterpretation of prevailing curricular theories and considered his own theory about the nature of curriculum to be quite distinct from the others (Kliebard). Dewey disagreed with the developmentalists regarding the extent to which the child should steer the curriculum. Where the 
developmentalists advocated for a curriculum driven by the interest of the child, Dewey called for more of a balance between attending to the desires and needs of the child and forging ahead with a specified curriculum that provides structure and guidance (Kliebard) in order to maximize learning.

Dewey's (1902) viewpoint on curriculum diverges from the other previously mentioned curricula theories (i.e. Social Meliorsim, Social Efficiency, and Humanism); however, he particularly took issue with the work of the Social Efficiency educators. In fact, Kliebard (2004) refers to the Social Efficiency movement as the antithesis of Dewey's ideas relating to curriculum reform. The Social Efficiency educators focused on creating a smoothly operating society by insisting on standardization in order to make the business of schooling more efficient (Kliebard). Social Efficiency reformers believed the role of curriculum was to prepare students "specifically and directly for the role they would play as adult members of the social order" (Kliebard, p.76) calling for "education according to predicted social and vocational role" (p.84). Dewey rejected this idea and believed that curriculum should facilitate the process of helping the child develop the necessary skills and dispositions to have an intellectual command of the modern world (Kliebard). In other words, Kliebard explains that Dewey acknowledged the importance of ensuring that children would be able to function as productive members of society; yet, he also supported the need to respect the way children see the world. While acknowledging the importance of teaching content, Dewey rejected the notion that the content should be standardized to the extent the Social Efficiency educators supported in the effort to control societal outcomes. 
The divergent viewpoints regarding whether curriculum should be systematic and standardized or contingent upon the particular learners involved are mirrored in the "Great Debate" (Chall, 1996) over what methods of reading instruction are most effective. Dewey's perspective on the significance of the child in the curriculum is a necessary reminder that there should be balance between teaching the content and regarding the learner despite the tendency of scholars to take sides and proclaim one as more important than the other. With the prevalent use of scripted curricula as an outcome of NCLB, I believe culturally and linguistically diverse students are not experiencing enough of a balance between the map that guides the curriculum and attention to the knowledge and unique experiences that they can apply to their learning.

\section{Reading Instruction Under NCLB}

Scripted reading programs are not new (Dewitz et al., 2010; Duncan-Owens, 2009); however, Duncan-Owens points out that, "the requirements of No Child Left Behind and the Reading First initiative have given commercial reading programs prominence in schools as principals look for ways to improve reading instruction through implementation of scientifically based reading methods" (p.26-27). Teachers are expected to implement scripted programs with fidelity (Duncan-Owens), which translates to:

Reading instruction where the commercial reading program, not the classroom teacher, determines what the teacher says during instruction and/or the particular lessons and the pace at which the lessons are taught (e.g., so many lessons taught in so many days). The teacher's role is to execute the plan of the commercial program without making adjustments for the instructional needs of the children in the classroom ("Scripted reading instruction," n.d., http://edresearch.info/scripted.asp). 
The belief that scripted programs should be used as the definitive map of how instruction and learning should play out, is reminiscent of curriculum theories supported by the Social Efficiency educators and the Mental Disciplinarian perspective (Kliebard, 2004). Overt focus on standardization, efficiency, and repeated practice diverge from the idea that instruction should be informed by the students who are trying to make sense of the content and assimilate new information into their existing knowledge. The desire to follow a particular protocol for success creates many positive outcomes; yet, at the same time the value that students bring to the equation can be lost. Strict adherence to a script does not reflect the balance called for by Dewey (1902), which he claims is necessary in order for students to find instruction meaningful and pertinent to their lives.

The obligation teachers face to teach a scripted program with fidelity using only the prescribed material juxtaposes the research in the area of culturally responsive teaching instruction, which calls for incorporating instructional strategies that draw upon what experiences and knowledge students contribute to the classroom (Gay, 2010). In the following section, I synthesize literature in the area of culturally responsive instruction in order to clarify what this type of instruction can look like in the classroom and how such instruction impacts culturally and linguistically diverse students.

\section{Culturally Responsive Instruction}

In order to illustrate examples of culturally responsive instruction, I will reference studies that incorporate empirical descriptions of teachers' actions in the classroom from Morrison, Robbins, and Gregory Rose's (2008) review of 45 classroom-based research 
studies, which utilized Ladson-Billings' (1995) three central tenets as an organizational framework for examining culturally responsive teacher practices. Ladson-Billings' tenet of cultural competence includes teacher actions such as reshaping the prescribed curriculum, building on students' funds of knowledge, and encouraging relationships between school and communities, which are directly related to my concerns regarding the implications of instruction that is based entirely on a prescribed core program. I considered all of the brief descriptions of the studies Morrison et al. included in their analysis and selected examples from their work that specifically address cultural competence per their coding. I further limited the studies analyzed by Morrison et al., in order to focus specifically on studies pertaining to culturally relevant teaching in reading instruction at the elementary level. Their work and reference list was a useful tool in order to locate other studies pertaining to culturally relevant reading instruction, which I will also reference.

The studies that I read describing examples of culturally responsive reading instruction shared three main themes. First, teachers utilized culturally appropriate texts and literature; second, they created learning environments where students' ideas, stories, values, and opinions were an integral part of the learning process; third, they made connections between their instruction and students' lives and students' funds of knowledge. According to the studies that I read, these three instructional strategies contributed to students feeling more connected to the curriculum and more engaged in their learning. 


\section{Culturally Appropriate Texts}

Several of the studies that I read focused on the inclusion of culturally relevant texts as an instructional tool to improve comprehension and engagement among linguistically and culturally diverse students (Bell \& Clark, 1998; Hefflin, 2002; LadsonBillings, 1992b; Powell, 1997). Teachers in these studies selected texts that were written by African American and Hispanic authors because the authors' cultural backgrounds and/or races matched those of their students (Ladson-Billings, 1992b; Powell, 1997). Teachers also took the time to select texts that included African-American characters and themes reflective of their students' lives (Bell \& Clark, 1998; Hefflin, 2002) and texts that "reflected the best elements of a cultural group's identity and practice" (Hefflin, 2002, p.234). Hefflin, for example, studied the process of collaboration between two teachers who worked together to plan a culturally responsive literature lesson for AfricanAmerican students. After observing a lesson on the text Cornrows, which positively highlighted and accentuated the lives of the children in the classroom, the author reflected that the students were more engaged with the culturally relevant lesson which was evident in more active participation in pre-reading activities, more elaborately written responses about their lives and their families, and longer engagement in richer discussions about African-American heritage and tradition. A quantitative study by Bell and Clark (1998) that compared comprehension results for groups of students that were exposed to texts that both did and did not align with their own racial identities also found that, "culturally sensitive reading material" (p.473) positively affected reading comprehension among African American children. 


\section{Engaging Student Voice}

In the literature that I reviewed, teachers also fostered a collaborative teaching and learning environment where students were able to share their ideas, tell their stories, and contribute to discussions that were open to an active dialogue among all participants (Howard, 2001; Hefflin, 2002; Powell, 1997). The teacher from Hefflin's (2002) study geared pre-reading discussion questions towards her students' African American heritage, called on students individually to share their ideas during a read aloud, and engaged in "dialogue journals" (p.244) with her students. She also facilitated a group discussion where she had students respond first and asked students to share portraits from their dialogue journals with the rest of the class. Both the teacher and author of this study realized the importance of thinking about pedagogy from different cultural perspectives and providing students opportunities to bring their cultural knowledge into the discussions. As a result of students' having many opportunities to share their own ideas and personal stories, their learning experience was enhanced and they more actively participated. Powell's (1997) longitudinal case study of culturally relevant teaching describes the teaching practices of a teacher who "often asked her students to write stories about their personal lives" (p.474) and "affirmed their many languages" (p.477) in order to make their voices heard. Another teacher taught her students that "good books are tied to authors' experiences" and asked her students to name topics they felt qualified to write about in order to affirm for them "the fact that they had a wealth of knowledge stemming from their personal and cultural experiences" (Ladson-Billings, 1992b, p.385). 
Additionally, Howard (2001), in a qualitative case study of 4 elementary school teachers, describes the use of communication styles in classrooms that were consistent with the communication styles used in students' homes so that students "didn't experience a cultural discontinuity between home and school" (p.190). One teacher incorporated a "morning circle" (p.191) so that students could talk about current events, issues, and whatever was happening in their lives and recognized that many of her students preferred oral communication, which is why she made sure to incorporate it into the daily classroom routines. Some of students in Howard's (2001) study indicated that they preferred teachers who allowed them to "actualize their own ideas" in assignments and group discussions (p.133). This study also indicated that students felt like their teachers interacted with them in ways consistent with how their parents and family members communicated with them. When interactions between students and teachers were conducted in a familiar cultural context, the students were more likely to behave in accordance with the teachers' expectations. The study concluded that students felt more comfortable with their teachers because of their "methods of communication, modes of interaction, and overall cultural knowledge" (p.145).

\section{Incorporating Funds of Knowledge}

A consistent theme among the studies read was the facilitation of connections between school content and students' lives through lessons that draw upon what knowledge students bring to the classroom. Moll et al. (1992) encourage educators to develop a deeper level of understanding about their students' cultures and families, which are referred to as students' funds of knowledge. They support viewing students' homes 
as, "rich in funds of knowledge that represent important resources for educational change" (p.139) and suggest that familiarity with these funds of knowledge allows teachers to facilitate a more "participatory pedagogy" (p.139) that draws on meaningful elements and activities of students' lives. Several of the teachers whose instruction was analyzed used the strategy of relating their lessons to students' lives (Benson, 2003; Powell, 1997). Consistent with Moll et al.'s understanding of funds of knowledge, one teacher visited students' homes in order to gain insight into their lives. She continuously explored students' cultural backgrounds and families and linked students' backgrounds to school culture (Powell, 1997) in order to reshape her curriculum to better align with students' backgrounds. She also felt it was important to reshape the prescribed curriculum and to provide students with differentiated assignments including different choices in order to best meet their needs. Finally, this teacher allowed her students to participate in shaping the classroom environment to make it more reflective of their homes, which resulted in her students bringing in a giant Mexican rug to tack on the wall. Because the concept of funds of knowledge is so integral to teachers being able to make curricula more culturally appropriate for linguistically and culturally diverse students, I have also included an entire section that reviews the literature in this particular area.

The teachers who were mentioned in the studies that I reviewed utilized culturally appropriate texts, established classroom communities in which students were encouraged to share their ideas as well as bring forth their values, and connected instruction to students' backgrounds and home lives which were all considered culturally responsive teaching strategies that led to positive outcomes for culturally and linguistically diverse 
students. As a result of their efforts to make their reading instruction more culturally responsive, their students had positive learning experiences in which they felt connected and motivated to learn. These studies provide clear, grounded examples of what culturally responsive instruction can look like in terms of early reading instruction.

The majority of the studies I read were qualitative and were based on case studies, interviews, and observations of teachers who were considered to be culturally responsive or teachers who were adjusting their teaching practices in order to reflect more culturally relevant pedagogy. I had a difficult time locating studies that utilized quantitative methodologies with experimental and control groups or correlations. I assume this is because culturally relevant instruction seems to be approached from constructivist and critical theory research paradigms due to the emphasis on cultural values and constructed experiences of different minority student groups. I believe this represents a gap in the research literature pertaining to culturally responsive reading instruction for diverse learners. Future research needs to weave together the valuable information gleaned from studies on culturally responsive pedagogy, the conclusions from literature in the area of reading instruction, and the literature focused specifically on instruction for culturally and linguistically diverse students, in order to investigate, through experimental and correlational research, what practices will positively impact student achievement and student learning. Culturally responsive teaching practices need to be used in conjunction with the platform of research-based scripted core reading programs that align with state standards and fulfill the mandates put forth by NCLB. 
For the purpose of this study, I have chosen to focus on funds of knowledge as a key component of culturally responsive instruction. In the next section, I will review literature relating to funds of knowledge and the process of conducting home visits in order for teachers to gain access to students' funds of knowledge. Additionally, the next section addresses how visiting their students at home affected teachers' perception of culturally and linguistically diverse students and their families.

\section{Discovering Funds of Knowledge}

Once I identified the concept of funds of knowledge as being integral to my interest in better meeting the needs of culturally and linguistically diverse learners, I began to seek out literature that would help me understand what actions teachers took to learn about their students' funds of knowledge. When using funds of knowledge as a search term, I was not successful in locating many articles or studies on this topic. I found more success when I used the thesaurus tool in the search engine because it was through this function that I discovered some related terms, one of which was home visits. In reviewing the literature on home visits in addition to exploring some other related search terms such as "schema" and "prior knowledge," I realized the distinction between what I would call background knowledge, or students' lived experiences (Vogt \& Echevarría, 2008) and funds of knowledge, which encompass knowledge accumulated from the students' households and families. It is now my understanding that while a teacher can gain an understanding of a student's background knowledge directly from the child within a classroom setting, in order to become better acquainted with a child's funds of 
knowledge, a deeper level of inquiry needs to take place in terms of the teacher investing more time to get to know students and their families.

As previously mentioned, the term funds of knowledge comes from the work of Vélez-Ibañez (1988) which focused on describing the "historically accumulated and culturally developed bodies of knowledge and skills essential for household or individual functional and well-being” (Moll et al., 1992). Subsequent to the work of Vélez-Ibañez and Vélez-Ibañez \& Greenberg (1992), Gonzalez et al. (2005) describe extensive work on the Funds of Knowledge Project that provided a deeper look at how families' funds of knowledge were accumulated by ethnographic researchers partnering with teachers. The work done to research funds of knowledge is grounded in anthropology stemming from an interest in culture (Gonzalez et al.). However, Gonzalez et al.'s project emphasizes ethnographic researchers collaborating with teachers in order to incorporate students' funds of knowledge into curriculum and instruction. Gonzalez et al. indicate that funds of knowledge are distinct from the concept of culture. Many different definitions of culture exist within the field of anthropology as well as education; yet, the work of Veléz-Ibañez and Gonzalez et al. provide a clear definition for the concept of funds of knowledge, making it much more narrow than the broad concept of culture. Additionally, The National Center for Research on Cultural Diversity and Second Language Learning (1994) points out that the concept of culture is often represented by generalizations such as food, dances, folklore; yet, funds of knowledge is a distinct concept based on the idea of talking and visiting with families in order to learn specifically about their lives, their family history, and their strengths rather than subscribing to generalizations about 
particular cultural groups. This reminds me of what we refer to as "below the line" information in Courageous Conversations indicating information that is not assumed about someone, but specifically garnered by getting that person's perspective and speaking directly to them.

Home visits are not traditionally part of teachers' requirements, as teachers are not provided the time and resources to make visiting families feasible (Meyer \& Mann, 2006). Yet, the studies and related articles I reviewed that focused on home visits (Baeder, 2010; Boske \& Benavente-McEnery, 2010; Ginsberg, 2007; Lin \& Bates, 2010; Meyer \& Mann; Moll et al., 1992; Vélez-Ibañez \& Greenberg, 1992) consistently report positive outcomes for teachers who participated in home visitations. Although research on teachers' attitudes about home visits is limited (Meyer \& Mann), the research I read consistently indicates that the practice of home visits has the potential to alter teachers' habits of making negative assumptions about culturally and linguistically diverse students and their families. Additionally, home visits have the potential to greatly impact the educational experiences of culturally and linguistically diverse students due to teachers being much more equipped and better prepared to infuse curricula with information, content, and themes that may be more culturally relevant to students.

Most of the studies I reviewed that focused on home visits were qualitative studies that utilized teacher narratives, field notes, interviews, and observations (Boske \& Benavente-McInery, 2010; Gonzalez et al., 2005; Lin \& Bates, 2010; Moll et al., 1992). The concept of funds of knowledge was utilized as a theoretical framework for investigating how teachers and school leaders could redefine their roles to engage with 
the community (Boske \& Benavente-McInery) and to work with teachers to develop innovations in teaching that draw upon the knowledge and skills found in local households (Moll, et al.). Additionally, much of the research took an ethnographic approach focusing on, "describing and interpreting the learned patterns of values, behaviors, beliefs, and language of a culture-sharing group" (Harris, 1968 as referenced by Creswell, 2007). There are many different types of ethnographic research and although the studies I read did not claim a particular affiliation to any sub-category of ethnography, I would suggest critical ethnography is a good fit with the purpose of funds of knowledge research. Creswell gives the example that critical ethnography is used as a means to speak out against inequality and domination, which aligns with how the funds of knowledge framework supports that all people are competent and all people have knowledge to offer (Boske \& Benavente-McIntery) despite prevalent deficit thinking with regards to culturally and linguistically diverse students and their families. One study took a narrative inquiry approach to researching funds of knowledge with a focus on investigating the meaning of experiences (Boske \& Benavente-McInery), which again acknowledged the core purpose of funds of knowledge by validating the perspectives of culturally and linguistically diverse families.

The practice of home visits emerged in the research I read as the dominant manner in which researchers' and teachers' collected information that was considered students' funds of knowledge. Prior to discussing the outcomes these visits had on teachers' perspectives of culturally and linguistically diverse students and their families, 
it is important to acknowledge and reiterate some of the perceptions held by teachers and schools regarding these students and their families.

\section{Teachers' Attitudes About Home Visits}

Lazar (2011) discusses how student populations are becoming increasingly more diverse; yet, the teaching force in the United States continues to be predominantly White, middle-class, and from European heritage, representing the culture of power. Teachers' lives are often disconnected from those of their students (Baeder, 2010); yet, Lin and Bates (2010) assert that teachers can certainly learn how to work more effectively with culturally and linguistically diverse students. Vélez-Ibañez \& Greenberg (1992) indicate that unfortunately, many public schools employ a deficiency model with regards to culturally and linguistically diverse students, which underutilizes their household funds of knowledge as a legitimate resource for developing innovations in teaching and does not place emphasis on the importance of teachers getting to know culturally and linguistically diverse families on a more personal level. This deficit perspective positions certain households as poor in terms of the quality of the experiences for the child (Moll et al., 1992), therefore teachers who are unfamiliar with the concept of funds of knowledge may not realize the extent to which familiarizations with students' households can positively impact their instructional practices and educational experiences for culturally and linguistically diverse students (Schmidt \& Lazar, 2011). The approach taken by Gonzalez et al. (2005) strives to, "re-present households in a way that is respectful to issues of voice, representation, and authenticity" in order to "represent communities in 
terms of resources, the wherewithal they possess, and how to harness these resources for classroom teaching" (p.x).

Only a few of the studies I read focused specifically on teachers' attitudes about conducting home visits; yet, as a result of the way in which the studies were conducted, teachers' reflections were a significant part of the data regardless of whether or not teacher attitudes about home visits were the main focus. Many of the descriptions of teachers' perceptions and thoughts about culturally and linguistically diverse students prior to actually participating in home visits align with the deficit thinking as mentioned by Moll et al. (1992) and Vélez-Ibañez \& Greenberg (1992).

Prior to participating in home visits, some teachers in Lin and Bates' (2010) study expressed concerns about the prospect of visiting students' homes indicating they were fearful of how parents would react to them visiting. Other teachers expressed fears about their personal safety when considering visiting students' homes such as Pauline who feared being robbed as well as Joyce and Pauline who expressed fears about being shot (Boske \& Benavente-McInery, 2010). Boske and Benavente-McInery note that all teachers who participated in their study shared misconceptions about of students and families, "owing to race, poverty, language, and immigration status" (p.379), which were all viewed as problems. Many of the teachers involved in the study placed blame on families such as Timothy who claimed he attended a home visit in order to, "show parents how bad their kids really are" (p.379) and Jessica, who identified students problems as "bigger than herself” (p.379). Additionally, many pre-service teachers who were asked to conduct home visits as part of an assignment during their student teaching 
were anxious about visiting students' home expressing skepticism about how the assignment would help them when they are in the classroom (Peralta-Nash, 2003). The author notes that principals at the school where these student teachers were placed and the cooperating teachers supervising the student teachers shared skepticism about home visits indicating that it would be dangerous to visit certain neighborhoods and that they would not invite themselves to the homes of students (Peralta-Nash). Many of these attitudes expressed in Peralta-Nash's study reflect the belief that, "students' home lives were to be left at the school gate" (p.119).

These concerns and hesitations are reflective of the deficit thinking that steers educators away from attempting to get to know culturally and linguistically diverse students and their families on a more deeper and personal level. Yet, despite the concerns they expressed prior to visiting homes, teachers' perceptions after getting to know students and families within their households were positively impacted (Boske \& Benavente-McInery, 2010; Meyer \& Mann, 2006; Peralta-Nash, 2003). In the following section, I will illuminate some of the more positive perspectives teachers expressed after participating in home visits and gaining a better understanding of culturally and linguistically diverse students and their families.

\section{Teachers Perspectives After Home Visits}

Despite concerns, fears, and negative thinking prior to participating in home visits, the results of the studies I read indicate that many teachers had very positive reactions to the experiences they had during the home visits and the interactions with their students and the students' families (Boske \& Benavente-McInery, 2010; Lin \& 
Bates, 2010; Meyer \& Mann, 2006). Teachers were exposed to poverty and home environments that left them thinking families were dirty, irresponsible, or illegal; yet, they still acknowledged how increased cultural awareness gained from the home visits affected their ability to examine their role as educators and understand their students and their families in new, distinct ways (Boske \& Benavente-McInery).

Teachers who participated in Boske \& Benavente-McInery’s (2010) study felt that home visits provided them opportunities to "deepen their empathetic responses and connect with the community at large" (p.382). The process of relinquishing the role of the expert in order to truly become a learner within students' households also allowed teachers to better understand "the rich cultural and cognitive resources of each household" (p.383) and develop meaningful relationships with families that they did not have previously. One of the conclusions made in this study was that building bridges between home and school has a positive influence on students' academic and language achievement, which contributed to teachers becoming invested in the process (Boske \& Benavente-McInery).

In Meyer and Mann's (2006) study, 26 teachers of grades K-2 visited the homes of 363 students. The authors note that when questioned about the home visits, teachers' responses included positive outcomes such as improved communication with parents, better understanding of the child, parents, and/or their personal situation, better understanding of the child's behavior, a different perspective on the child's strengths and challenges in the classroom, and increased rapport and trust between the teacher and the parent. Some of the teachers who participated in this study also reported increases in 
average daily attendance, an increase in the number of parents attending conferences, and greater family participation in school activities among families who were involved in home visits (Meyer \& Mann). One teacher commented that participating in a home visits made her more understanding of students who are not offered help at home and therefore she was able to make time during the school day to provide students with the support they needed. Finally, $100 \%$ of teachers who participated stated they planned to do home visits again the following school year (Meyer \& Mann).

Lin \& Bates (2010) note that all participants in their study found that their attitudes toward families of different backgrounds became more compassionate and empathetic as a result of participating in home visits with their students. Teachers in this study also reported the desire to bring their students' cultures into the classroom as much as possible and to spend more time building respect for all cultures. One teacher from this study remarked, we seem to fear the unknown, knowing someone and understanding some things about them keeps us from being part of that fear" (Lin \& Bates, p.184). This statement concisely summarizes the distinct power of visiting students' homes in order to bypass the fears associated with broad generalizations about culturally and linguistically diverse students that result in deficit thinking.

However, despite the potential benefits for teachers, students, and families that can be derived from home visits, home visits are not a widely used practice (Meyer \& Mann, 2006) in elementary schools as a means for teachers to deepen relationships with students and their families. The previously mentioned studies include data and feedback from elementary teachers; yet, the practice of home visits is not widespread enough, and 
not enough studies have been done focusing on home visits at the elementary school level, to provide substantive data regarding the positive effects of home visits for elementary aged children and their families.

\section{Culturally Responsive Instruction with Scripted Curricula}

Literature pertaining to the concept of culturally responsive instruction is predominantly situated in constructivist teaching scenarios in which teachers have a high degree of control over curriculum creation and implementation. However, many contemporary teachers are not provided such freedom and are mandated to use prepackaged commercial programs that are scripted (Fang, Fu, \& Lamme, 2004; Peck \& Serrano, 2002). When considering the process of utilizing information about students and their families to make curricula more relatable or engaging for them, it follows that teachers who have more freedom to create and design their own lessons or content units would have more opportunities to teach in a culturally responsive way.

Given the prevalence of prepackaged commercial programs, I found myself more interested in literature and studies pertaining to the use of culturally responsive instruction and teaching strategies within the context of the implementation of scripted curricula. Searching scripted instruction and related terms, however resulted in a lot more literature calling for implementation of scripted programs with fidelity as opposed to examples of how such programs can be modified, altered, changed, or enhanced or suggestions that such programs should be changed at all. Fortunately, I was able to find a few studies that address modifying scripted curricula that relate to my intended research and highlight the gaps in the literature that I believe need to be addressed. 
Eisenbach (2012) identifies three different approaches (i.e. the accommodator, the negotiator, and the rebel) teachers took when implementing a scripted language arts curriculum that did not align their personal teaching ideologies. The accommodator used the scripted program as the primary source of instruction despite not agreeing with all of the activities, which was evidenced by "scripted posters, student-generated scripted work, a scripted word wall, and a bulletin board containing 'unpacked' scripted assessment" (p.154). The rebel teacher, conversely, simply did not do the scripted curriculum at all. Instead, he utilized lessons, activities, and novel studies he designed himself defending his actions by saying, "my students learn and they score well on standardized assessments" (p.156). Finally, the negotiator "infused her own ideas and beliefs into a prepackaged agenda" (p.154) with strategies such as utilizing terms from the curriculum on the classroom word wall as well as terms from supplementary classroom content and having students complete their own mini-booklets as opposed to completing a lesson on paper in standard writing format. This teacher found ways to compromise by picking and choosing the lessons/activities that would benefit her students the most and filling the "non-scripted gaps" with her "own items" (p.155). In considering how teachers incorporate culturally responsive lessons while implementing scripted curricula, it remains to be seen which approach they emulate in order to do so.

Based on much of the literature I read, I found the notion of culturally responsive instruction to be distinct from and unrelated to the implementation of scripted instruction. This mutual exclusivity would lend itself to the assumption that teachers would have to employ the attitude of the rebel and completely diverge from scripted curricula in order to 
teach in a culturally responsive way. Yet, efforts to "negotiate" culturally responsive instruction into the use of scripted curricula were apparent in a few of the studies that I read.

Peck \& Serrano (2002) examined the effectiveness of Open Court, a scripted language arts curriculum, with ESL children through eliciting the perspectives of approximately 100 teachers who were education students at California State University Northridge, supervising six student teachers, and examining Open Court manuals and decodable books. The authors describe Open Court as a teacher proof language arts curriculum that includes prescribed materials and a script to be followed. Yet, in their study they did observe lessons in which the teachers adapted the curriculum to meet the needs of the children. The authors indicate that some experienced teachers were able to "tweak" Open Court in order to make it more effective for ESL students. Some of the ways in which teachers did so were by brining in realia and guest speakers to build background knowledge for a lesson, by allowing students to pose their own questions and story predictions rather than those indicated by the teacher's manual, by making vocabulary activities that students see or hear in their environments, and by teaching the lessons at a slower pace than indicated in the teacher's manual. The study also indicated that teachers needed to plan their own lessons to build background knowledge and those lessons then took time away from the lessons in the curriculum.

Although this study addressed the idea of tweaking a scripted program in order to make it more appropriate for English Language Learners, the examples of what teachers did and how they went about "tweaking" the program were vague and did not supply 
sufficient detail to provide a model for replication. The authors concluded with additional questions such as, "What is the range of ways in which teachers follow or alter the lessons, with the blessings of their principals and coaches?" and "What portraits can we paint of expert and effective teachers who use Open Court (e.g., strategies, techniques, ways to follow and not follow the teacher's guide?" (p.15). These are some of the same questions that I have regarding precisely how teachers might enhance scripted curricula. Another study by Fang, Fu, and Lamme (2004) describes “a longitudinal professional development project designed to support the efforts of in-service teachers to make pedagogical transitions from total reliance on prepackaged commercial programmes to making informed decisions about curriculum and pedagogy autonomously" (p.58). The study involved four teachers who agreed to create professional development classrooms (PDCs) and who were provided a support structure by means of a) an annual summer institute, $b$ ) regular classroom visitations by university faculty, staff members from the North each Florida Educational Consortium, and fellow teachers, c) monthly meetings throughout the school year, and d) the end-of-year showcase meeting for the purpose of empowering them to make informed pedagogical decisions. From participating in such structured and thorough professional development, the PDC teachers learned to "trust their own professional wisdom and judgment based on their daily observation and interaction with students" (p.61). One teacher reflected, "In the past I just followed the textbooks, now I design my teaching and choose what I want to teach according to what my kids need" (p.61). 
It is important to note, however; that teachers participating in this study were given exemption from textbook adoption, meaning they were not required to utilize the adopted textbooks as other non-PDC teachers were expected to. Consequently, in order to instruct in ways that better addressed the needs of the students in their particular classes, teachers moved away from scripted instruction and began to make more instructional decisions themselves rather than referring to a textbook or commercial program. Additionally, the main focus of the study was to investigate the effect a complex model of professional development had on teachers' ability to make pedagogical decisions more independently and how students were affected as a result. Therefore, once again, despite addressing the need for "teachers to make informed, flexible and creative uses of available instructional resources rather than adhere to a particular programme or dogma" (p.64), the study did not explicitly describe specific pedagogical decisions teachers made in their classrooms to make instruction more suited to their students. Nor did it address the extent to which PDC teachers used textbooks or commercial programs, if at all, in conjunction with their instruction. With regards to my interest in the interplay between scripted curricula and culturally responsive instruction, this study also leaves many questions unanswered pertaining to specific strategies, methods, or tactics teachers are utilizing in order to weave culturally responsive instruction into the use of a scripted program.

Kavanagh (2010) conducted a holistic multiple case study for which the research questions share common ground with my study. Kavanagh's study investigated how two female $4^{\text {th }}$ grade teachers negotiated their culturally relevant teaching ideologies with the 
mandate to utilize Success For All (SFA) language arts curriculum with fidelity. Primarily through interviews, classroom observations, and observation debriefs, the researcher found that the teachers Dee and Fiona supplemented the scripted program and skipped certain parts of SFA in order to modify their instruction to better align with their culturally relevant teaching ideologies.

The teachers in Kavanagh's (2010) study felt that simply following SFA as it was intended did not result in instruction that was rigorous enough; yet, they acknowledged the importance of sticking with the main idea of what they were supposed to teach and then making it more challenging and more culturally relevant through supplements. One of the teachers spoke of a "balance between doing what they tell me to do and then adding things that the kids relate to," emphasizing that "you can incorporate and infuse your own ideas but still do what they are asking you to do" (p.113). The teachers reflected on sticking to the script completely when they first started using it, then feeling more competent to modify the scripted program as they became more experienced and familiar with the content. In order to make instruction more culturally relevant, Dee and Fiona added more opportunities for their students to engage in critical and higher order thinking and also made efforts to connect to students' identities by bringing in pamphlets, outside readings, video clips, and articles so students would have something to relate to. Additionally, Dee and Fiona tried to "craft opportunities for kids to be known, seen, valued, and contributory to the learning of others" (p.136). The teachers, however, felt constrained by many factors, including the mandate to use SFA according to the script, therefore they made "subtle attempts" to infuse culturally relevant pedagogy during the 
scripted instruction and incorporated culturally relevant instruction to a greater extent outside the 90 minute SFA block when they had "the freedom to do so."

Although this study shares significant common ground with my study, there is one particular difference that is noteworthy in terms of a unique contribution my study addresses. Dee and Fiona were both African American and the populations of students in their schools were 96\% and 99\% African American respectively. Culturally responsive instruction does not become less pertinent if the teacher shares the same race or ethnic background as the students; however, when the teacher does not share the same race or ethnic background as the students, there may be a much larger disconnect between the lived experiences of the teachers and those of the students. It is with that in mind that my study sought to better understand how teachers (within a district where the teaching force is predominantly White) go about better understanding their students who are culturally and linguistically diverse to the extent that they could modify or enhance instruction in order to make connections to their students' lives and lived experiences. I do think however, the idea of becoming familiar with students' funds of knowledge as part of culturally responsive instruction depends getting to know specific information about specific students rather than making generalizations about students based on their racial, cultural, or ethnic affiliations whether shared between students and teachers or not. For this reason, my interest in understanding the ideas and behaviors teachers have regarding how to better understand their culturally and linguistically diverse students still addresses a gap in the existing literature. 
After previously having difficulty locating literature addressing culturally responsive pedagogy in the context of scripted instruction, I was excited to come across the aforementioned studies that are not situated in a constructivist context. However, despite encountering some literature that aligns much more closely with the teaching context I have experienced, I still believe that the overall body of literature in this specific area is lacking. There are gaps in the literature that leave me wanting more details and examples of how teachers negotiate the relationship and experience the interplay between culturally responsive pedagogy and the implementation of scripted curricula. It is my hope that my research will contribute to filling that gap.

\section{Conclusions}

In the attempt to synthesize and fit together the various components of my literature review including curriculum theory, reading instruction under NCLB, culturally responsive pedagogy, and accessing students' funds of knowledge, I recognize a gap in the literature that separates the ideals ingrained in culturally responsive instruction and the way instruction is carried out in classrooms as a result of NCLB and the prevalence of scripted curriculum. I believe that teachers have the potential to greatly enhance scripted curricula by infusing prescribed lessons with instructional activities and strategies that relate to culturally and linguistically diverse students' funds of knowledge; yet, the current literature does not provide sufficient descriptions or resources that would provide teachers with a clear understanding of how to do so in a way that maintains the fidelity of the scripted program. Because culturally responsive instruction is a very subjective endeavor, there will not exist a specific set of practices or directions for modifying 
scripted content to better reflect the experiences of culturally and linguistically diverse students. Teachers who are intentional about becoming culturally relevant educators will have a significant undertaking in order to know culturally and linguistically diverse students and become familiar with the students' funds of knowledge in such a way that allows the teachers to enhance scripted curricula based on what they discover about their students. Yet, more detailed descriptions of how teachers have succeeded in negotiating the relationship between the implementation of scripted curricula and their intentions to be culturally responsive will make an excellent contribution to the existing body of knowledge about current educational practices. My study provides rich examples of how my participants, who implement scripted curricula, have engaged themselves in an explicit focus on creating culturally responsive lessons focused on better meeting the needs of culturally and linguistically diverse students.

The body of knowledge pertaining to home visits supports the practice of visiting students' homes as a very productive avenue for teachers to learn more and better understand culturally and linguistically diverse students and their families. However, because home visits are not required or a widespread practice among elementary teachers, I was interested in discovering what ideas and behaviors teachers have developed for becoming more familiar with their culturally and linguistically diverse students and their families in order to be acquainted with their funds of knowledge. I gained a better understanding of how teachers go about becoming familiar with their culturally and linguistically diverse students' funds of knowledge, how those funds of knowledge were used to create culturally responsive instruction, and how teachers wove 
culturally responsive instruction into scripted curricula they are expected to implement with fidelity. 


\section{CHAPTER III}

\section{RESEARCH AND METHODOLOGY}

Chapter three serves as an outline for understanding my research goals and how I carried out my research study. In this chapter, I will identify my research paradigm (i.e. constructivism/social constructivism) and the theoretical framework (i.e. funds of knowledge) on which the study was based. I include descriptions that contextualize my study in terms of who participated, how I collected and analyzed data, and how I used the data from the study to inform and connect the bodies of literature related to my research questions.

\section{Research Questions}

The research questions explored in this study were:

- R.Q.1 How do teachers who implement scripted curricula describe the experience of creating culturally responsive lessons intended to specifically connect with their culturally and linguistically diverse students' funds of knowledge?

- What do teachers report about the ways in which they draw from or incorporate students' funds of knowledge in their instruction?

- How do teachers perceive the impact of culturally responsive instruction for culturally and linguistically diverse students in their classrooms?

- R.Q.2 What do teachers report regarding the ideas and behaviors they have developed as ways to better understand their culturally and linguistically diverse students and their families? 
- What do elementary teachers report about factors that influence their willingness to conduct home visits as an avenue to better understand culturally and linguistically diverse students and their families?

The goals of this study were to investigate how and what teachers employ to adapt the prescribed curriculum for culturally and ethnically diverse students. Additionally the study investigated what behaviors and/or ideas teachers have for becoming familiar with students' funds of knowledge. The study was designed with the purpose of contributing to the knowledge base regarding how the seemingly separate bodies of literature pertaining to both implementation of scripted curricula and the creation of culturally responsive instruction can be combined to elicit more equitable and effective instruction for culturally and linguistically diverse students.

\section{Research Paradigm}

Based on my commitment to valuing the funds of knowledge that all families have to offer and my investment in enhancing scripted curricula with culturally responsive instruction that incorporates students' unique funds of knowledge, the constructivist research paradigm closely aligns with my goals as a researcher. The constructivist paradigm values multiple constructions of reality (Guba, 1990) and the acknowledgement and appreciation of multiple perspectives are integral aspects of funds of knowledge and culturally responsive instruction (Gay, 2010). Guba notes that according to the constructivist research paradigm, reality is relative, meaning there is not an ultimate truth that can be discovered through inquiry. This notion makes individual constructions of reality pertinent even if various interpretations do not lead to a single 
conclusion. I would like to better understand how teachers utilize students' funds of knowledge to enhance scripted curricula in order to make it more appropriate and engaging for culturally and linguistically diverse students. As I continue to learn about culturally responsive instruction it becomes increasingly clear that there is not a specific formula or list of best practices for making curriculum culturally responsive. On the contrary, making curriculum and instruction culturally responsive is a unique endeavor for each teacher that attempts to do so because culturally responsive instruction is closely tied to specific students in particular classrooms and their unique experiences and funds of knowledge. This research focused on teachers' experiences of enhancing curriculum in order to better reflect their particular students and focused on understanding their individual perspectives about the process and subsequent outcomes in terms of their teaching and their students' learning.

More specifically, I approached this study from a social constructivist perspective because I acknowledge that my own experiences and background influenced how I made sense of and interpreted the meaning of participants' responses (Creswell, 2007). Given that I am also a teacher who is trying to create culturally responsive lessons that attend particularly to my culturally and linguistically diverse students' funds of knowledge, I deeply reflected on the questions that asked my participants. I acknowledge that my role as the researcher coincided with my role as a classroom teacher and therefore I continued to reflect and adjust my own teaching practices as I collected data and analyzed the data derived from the participants. I have common ground with the teachers who participated in the study in terms of our shared experience as members of our schools' Collaborative 
Action Research for Equity (CARE) teams, so it was my intention to use my own ideas, experiences and interpretations as a means to add to the depth and clarity of the themes and conclusions yielded by the data from the study. I was purposeful, however, in ensuring that my own experiences did not interfere with my ability to analyze participants' data without bias.

\section{Theoretical Framework}

Vélez-Ibañez's (1988) concept of funds of knowledge serves as the theoretical framework on which this study stands. The conviction that all people are competent, all people have knowledge, and people's life experiences give them that knowledge (Boske \& Benavente-McEnery, 2010) is the foundation on which I have established my own theory that teachers can greatly enhance the impact of scientifically research-based curricula by blending it with culturally responsive instruction that draws upon culturally and linguistically diverse students' funds of knowledge. Although the current bodies of literature do not represent a synchrony between instruction driven by scripted curricula and culturally responsive instruction, I believe that teachers who employ reflective practice and make efforts to become more familiar with their culturally and linguistically diverse students can successfully enhance scripted curricula in order to provide for better outcomes for those students.

A variety of perspectives and ways of encountering the world must be represented in schools and therefore content instruction should reflect and relate to the variety of perspectives that are inherent among today's diverse classrooms. If teachers rely entirely on prescribed, scripted curricula it is more than likely that culturally and 
linguistically diverse students will continue to lag behind their White peers and experience school in a way that makes them feel disconnected. The evidence in literature pertaining to culturally responsive instruction needs to push teachers towards a higher level of reflection and evaluation with regards to scripted curricula in order to modify curricula in ways necessary for better connecting with culturally and linguistically diverse students.

\section{Overview of Research Methods}

This qualitative collective case study utilized individual interviews and a focus group as the primary means to investigate what teachers think and do in order to better understand culturally and linguistically diverse students. Additionally, the study investigated how teachers describe the process of creating culturally responsive lessons based on their students' funds of knowledge while also being held accountable for implementing scripted curricula.

\section{Context of the Study}

The school district I selected for my study serves more than 10,000 students near Portland, Oregon. For the purpose of maintaining confidentiality in this study, I will refer to the district as District CC. Approximately 13\% of students within District CC are categorized as English Language Learners, meaning they are in the process of learning and acquiring English language skills. Like many other school districts in the United States, District CC serves an increasingly diverse population of students and students of color. Both teachers and administrators are focused on improving student achievement particularly because certain subgroups of students, such as Latino students and ELL 
students have failed to meet annual yearly progress (AYP) targets in recent years. Lowincome students, English Language Learners, students with disabilities, and Latino students continue to be District CC's lowest achieving subgroups.

In addition to making efforts to ensure that all teachers within District $\mathrm{CC}$ are highly qualified and providing teachers with professional development opportunities to help improve their practice, District $\mathrm{CC}$ has invested a lot of time and resources into eliminating the racial achievement gap in order to ensure educational equity and excellence for all students. District CC enlisted the assistance of Pacific Educational Group as a partner in the systematic equity transformation within the district in order to focus professional development and teacher training explicitly on race and the role race plays in classrooms. Beginning in 2007, District CC began involving teachers in Courageous Conversations about Race and Beyond Diversity trainings with the goal of having all teachers trained in the Courageous Conversations protocols, which include four agreements and six conditions outlined in Table 3. 
Table 3

Courageous Conversations Agreements and Conditions from Singleton \& Linton (2006)

\begin{tabular}{|c|c|}
\hline Four Agreements & 6 Conditions \\
\hline 1. Stay engaged & $\begin{array}{l}\text { 1. Establish a racial context that is } \\
\text { personal, local, and immediate }\end{array}$ \\
\hline 2. Experience discomfort & 2. Isolate race \\
\hline 3. Speak your truth & $\begin{array}{l}\text { 3. Normalize social construction and } \\
\text { engage multiple perspectives }\end{array}$ \\
\hline \multirow[t]{3}{*}{ 4. Accept and expect non-closure } & $\begin{array}{l}\text { 4. Monitor agreements, conditions, and } \\
\text { establish parameters }\end{array}$ \\
\hline & 5. Establish a working definition for race \\
\hline & $\begin{array}{l}\text { 6. Understand the role and presence of } \\
\text { Whiteness }\end{array}$ \\
\hline
\end{tabular}

The protocols listed in Table 3 are utilized in various trainings that are part of Courageous Conversations as well as other professional development opportunities within District CC in order to establish expectations for conversations and conduct. The agreements and conditions are used with the intention of keeping an explicit focus on race and the district objective of reducing the racialized achievement gap.

Further, I chose to use District CC for this study because many teachers in the district participated in a series of CARE trainings during the 2011-2012 school year specifically focused on the creation of culturally responsive lessons that addressed their culturally and linguistically diverse students. At the beginning of the 2011-2012 school year, each school principal put together a team of teachers to participate in the series of trainings on culturally responsive instruction that occurred every other month of the 
school year. I am part of the CARE team from my school and therefore have firsthand experience with the exercises and activities that were included in the district's training. I have worked extensively with colleagues from my school to create culturally responsive lessons and have acquired a significant interest in how other teachers perceived the same experience as well as an acute interest in how other teachers engaged in the process of creating culturally responsive lessons.

At the initial training in the fall, teachers were asked to select 3-5 students of color to function as their focal students for the duration of the year. I use the term culturally and linguistically diverse students in my written work to align my verbiage with the literature I have read. However, I consider students of color, or focal students as they are referred to in my interview protocols, to be among the students I would describe as culturally and linguistically diverse. For the purpose of this study the terms culturally and linguistically diverse students and focal students will be used synonymously. Teachers were asked to collect below the line information about their focal students, which refers to information that comes specifically from the students about themselves and their families as opposed to information derived from assumptions about students based solely on their racial or cultural identity. At the outset of this study, I equated below the line information to the type of information about students and families referred to in the literature as funds of knowledge and therefore used those terms synonymously. A discussion about that decision and its implications for the study can be found in the final chapter. Teachers were also asked to document what engagement looks like for each 
student during instruction as a baseline for judging the effectiveness of the culturally responsive lessons that would be created as part of the training.

CARE team members were presented with a motivational framework for culturally relevant teaching summarized by four quadrants, or areas of focus, which are a) establishing inclusion, b) developing a positive attitude, c) enhancing meaning, and d) engendering competence (Table 4). Teachers were then asked to select and incorporate specific items from the framework (Appendix A) into culturally responsive lessons.

Table 4

Framework for Culturally Relevant Teaching

Establishing Inclusion
"Relationships"
A learning experience in which a
community of learners feels respected and
connected to one another

Enhancing Meaning "Rigor"

A learning experience that engages students in challenging ways

\section{Developing a Positive Attitude "Relevance"}

A learning experience that offers meaningful choices and promotes personal relevance

\section{Engendering Competence "Realness"}

A learning experience where students become more effective in learning they understand and value because it has authentic real world meaning

The information about focal students that the teachers collected and documented was incorporated in conjunction with the framework in order to create several culturally responsive lessons geared towards eliciting active engagement, participation, and achievement from the focal students.

In order to evaluate the effectiveness of the culturally responsive lessons that were created, CARE team members also participated in an observation process in which 
teachers from each team collaborated to observe each others' culturally responsive lessons. Teachers were asked to focus only on the focal students during observations and to provide the teacher conducting the lesson with notes and feedback about the engagement of that teacher's focal students during the lesson. The first time this observation process took place the entire CARE team, including the CARE facilitator and building principal, participated in observing each team member during the implementation of his or her culturally responsive lesson. Teachers were encouraged to then continue the observation process in a more casual format by arranging to observe one another on a more frequent basis without the entire group in attendance for each observation.

Teachers who participated on CARE teams in District CC were part of a fairly extensive process of receiving training in the area of culturally responsive instruction and creating culturally responsive lessons. I invited CARE teachers to participate in my study in order to learn about how they became more familiar with their focal students' funds of knowledge as well as their process of creating culturally responsive lessons particularly focused on enhancing the educational experiences and opportunities for those students.

\section{Participants}

I deemed five cases necessary in order to gather sufficient data and represent multiple perspectives that may have been experienced by teachers who participated in CARE training. I had hoped to secure six participants, which would offer a safeguard in the event any of the participants decided to discontinue participation in the study prior to completion; yet, I was only able to recruit five teachers for the study. Because I 
conducted a series of three in depth interviews with each participant, a sample larger than six cases would have presented logistical challenges in terms of scheduling interviews and managing the large amount of data that would accompany a larger number of participants and interviews. Using fewer than four cases would have resulted in data analysis challenges with regards to developing themes across cases.

In order to obtain the desired sample size, I initially provided information about the study to all teachers who participated in CARE training during the 2011-2012 school year via email (Appendix B) inviting them to contact me if they were interested in participating in the study. I also encouraged CARE participants to contact me if they had any additional questions that had not been answered with the information provided in the email. A letter of informed consent (Appendix C) was included with the email as an attachment, so all potential participants would be aware of the research purpose, potential risks, and participant expectations. Although the email describing the study was sent to all teachers who participated in CARE training, which included K-12 teachers, I indicated that I was looking for participants who teach grades K-5. Two principals responded to my email indicating they would be willing to help in any way they could; yet, I did not use them as participants because, although they were part of the CARE observation process, they did not create or teach culturally responsive lessons themselves.

Because I sent out my request for participants very close to the end of the school year, I was concerned that I would not be able to achieve the desired sample size as teachers have many other concerns and obligations as the end of the school year approached. I decided to send a follow-up email (Appendix D) including information 
about the study and the significance of the research goals shortly after sending the initial email so CARE participants would have another reminder about the opportunity to take part in the study relating to their CARE work. Also, just in case more than six teachers expressed interest in participating in the study, I prepared a survey (Appendix E) to administer electronically via Survey Monkey to function as selection criteria and to ensure maximum variation with regard to grade level and school, favoring schools that have greater populations of culturally and linguistically diverse students. The survey was not needed; however, as I only received responses from five interested teachers. The five teachers who expressed interested in participating in my study served as the cases for this collective case study. A sixth teacher emailed me shortly after the school year was over and expressed interest in participating; however, I did not get a response when I replied back.

\section{Methodological Approach}

I selected a case study as the most appropriate research method to answer my research questions. I selected a case study as the ideal methodology grounded on Yin's (2009) statement that, "You would use the case study method because you wanted to understand a real-life phenomenon in depth, but such understanding encompassed important contextual conditions - because they were highly pertinent to your phenomenon of study" (p.18). This statement made sense in the context of my research questions because the research questions for my investigation draw from specific experiences (i.e. collecting information about particular students of color they selected as focal students and creating culturally responsive lessons based on the information they 
gathered) teachers in District CC had as part of CARE training. All elementary teachers in District CC are expected to implement the scripted programs the district adopted; however, not all teachers within the district have specifically engaged in work pertaining to the creation and implementation of culturally responsive lessons. Teachers who have not been part of CARE training would not necessarily have the foundational knowledge pertaining to culturally responsive instruction and gathering information about culturally and linguistically diverse students to provide sufficient data with regards to the research questions. Therefore, the case study method was appropriate because it allowed me investigate and better understand teachers' perspectives about getting to know their culturally and linguistically diverse students and creating culturally responsive lessons within a given context of a shared experience (i.e. participation in CARE training).

A case is defined as a bounded system (Johnson \& Christensen, 2008), or the “object of study" (p.406). Creswell (2007) indicates that an individual, several individuals, a program, an event, or an activity can all serve as cases. Using these descriptions of what constitutes a case, I defined my intended units of analysis, or cases, as the individual K-5 elementary teachers who work in District CC that have participated in CARE training during the 2011-2012 school year. I chose individuals as cases rather than the overall process of CARE training because I wanted to know how each individual teacher utilized the activities assigned in CARE training (i.e. collecting below the line information about focal students and creating culturally responsive lessons based on that information) within the context of his or her own classroom and students. The CARE training provided a common experience and a context for the cases; yet, my interest was 
more focused on the individual teachers' perspectives and actions as a result of that experience than details about the experience itself. By designating individuals as cases, I was able to gather in depth data pertaining to the perceptions of, and actions taken by, each person in order to gain a better understanding about the potential interplay between culturally responsive instruction and scripted curricula. Because I anticipated that each participant's experience of gathering information about his or her focal students, creating culturally responsive lessons, and integrating cultural responsiveness with scripted programs would be unique, I chose to focus on each particular individual as a distinct case (Nieto, 2008).

Creswell (2007) describes three different types of case studies, which are the single instrumental case, the collective or multiple case study, and the intrinsic case study. I selected a collective case study because I agree with Herriott \& Firestone (1983) as cited by Yin (2009) that multiple cases are needed to make the study more compelling and robust. I believe that a collective case study was necessary in order to obtain a data set representative of different ways teachers interpreted and described the process of adapting instruction to better meet the needs of culturally and linguistically diverse students as well as different ways teachers accessed their culturally and linguistically diverse students' funds of knowledge. Even though the number of cases studied was not large, the study elicited sufficient data to contribute to a richer understanding of how teachers experienced using scripted curricula while at the same time attending to the cultural and racial differences among student populations in order to incorporate students' funds of knowledge into instruction. Johnson and Christensen (2008) indicate 
that multiple cases sacrifice depth for breadth; yet, I don't think one case would provide sufficient data to draw conclusions about the topic or generalize the results in order to contribute insight about the interplay between scripted curricula and culturally responsive instruction to the field of education.

When selecting cases for the study, I used what Creswell (2007) refers to as "accessible cases" because teachers became subjects based on their willingness to participate. Creswell also refers to ordinary cases and unusual cases; however, I did not have a way to classify the cases as either ordinary or unusual prior to collecting and analyzing data from the participants. Therefore, I collected data from cases that were accessible and then drew conclusions about the cases during data analysis. I also used replication of procedures (Yin, 2009) across all participants in order to have a basis for comparing the various cases.

\section{Data Collection}

Yin (2009) notes that a major strength to the case study design is the opportunity to use many different sources of evidence. I used several different data sources so that conclusions made from the data collected draw upon multiple sources of evidence. Data sources for the study included a) individual in-depth interviews, b) a focus group, c) artifacts from CARE training, and d) a research log.

\section{Individual Interviews}

Seidman (2006) suggests that a series of three interviews with each participant is most appropriate in order to contextualize the participants' experiences and additionally 
explore the meaning of those experiences. Seidman references Schuman (1982) to describe the purpose of each interview within the series noting that:

The first interview establishes the context of the participants' experience, the second allows participants to reconstruct the details of their experience within the context in which it occurs, and the third encourages the participants to reflect on the meaning their experience holds for them (p17).

Although Seidman uses this interview format as part of phenomenological research, I found it applicable to the multiple case study approach that I selected. I agree that interviewing a participant only one time would not necessarily allow time to develop a repertoire with that person in order to obtain data that not only contextualizes their experiences but that would also speak to the meaning behind those experiences and the participant's reflections pertaining to the experiences. A series of three interviews with each participant over a period of approximately five months facilitated a better opportunity to collect meaningful data pertaining to my research questions.

Because I did not conduct a phenomenological study, I used the series of three interviews format with adjustments made to the purpose of each interview making the sequence more appropriate for my particular research questions. This allowed me to focus each interview on a particular aspect of my inquiry and to spend a sufficient amount of time with each participant while she unpacked and described her experiences with creating culturally responsive lessons and becoming familiar with her focal students' funds of knowledge. The first interview focused on establishing what participants understood as the meaning of being a culturally responsive educator and how the participants became familiar with their students' funds of knowledge. The second 
interview allowed participants to reconstruct details of their experiences creating culturally responsive lessons as part of the CARE training process. Participants were encouraged to bring artifacts from CARE training to this interview such as notes, samples of their culturally responsive lesson plans, or their team's CARE story. Finally, the third interview allowed participants to reflect on the value of home visits. The third interview followed a focus group session centered on a discussion about home visits. When selecting participants I hoped to include participants who had conducted home visits, as well as participants who had not, in order to represent perspectives from both groups. Luckily, despite only having five teachers willing to participate in the study, they did represent a mixture regarding whether or not they had previously conducted home visits. Regardless of whether or not participants had personally conducted home visits; the focus group conversation provided an opportunity for all participants to think about the potential benefits and challenges that can be associated with home visits. The final interview then allowed each participant to share personal reflections about the idea of conducting home visits as an avenue to better understand their culturally and linguistically diverse students and connect instruction to those students' funds of knowledge.

I spent approximately 30-60 minutes with each participant during each interview and all interviews were guided by interview protocols (Appendix F). Although I intended to replicate questions across each case, I maintained flexibility in the flow of the conversations, bearing in mind that conversations with various participants would differ greatly and wouldn't necessarily provide for the same exact flow of questioning in the 
course of the conversation. Interviews took place in participants' homes, participants' schools, and my home, which provided a quiet, private atmosphere. All interviews were audio recorded using the iTalk app on an iPhone device and then transcribed verbatim. In order to organize transcripts, I assigned each participant a number, which was recorded at the top of the first page along with the date of the interview, the location of the interview, the ordinal number for that interview, and the time the interview took place. The iPhone and MacBook Pro used for recording and transcribing data remained in my possession or in my residence at all times so no one else would have access to the data or the files. Additionally, both the iPhone and MacBook Pro were password protected. All data was kept confidential and accessible only to my doctoral advisor and myself.

\section{Focus Group}

Once all of the participants completed the second interview, they were invited to come together for a focus group discussion centered on the topic of home visits. The intention of using a focus group as a source of data was to gather data that explicitly provided evidence about similarities and differences among participants' perspectives (Morgan 1996; Morgan, 1997) on the topic of home visits as opposed to aggregating data from individual interviews to make that comparison. A focus group provides an opportunity for the researcher to specifically ask participants to compare and contrast their experiences and/or ideas (Morgan, 1996), which is not an option in conversing with one participant at a time. One of the discussion prompts in the focus group protocol (Appendix G) directed participants to brainstorm some of the benefits and challenges associated with home visits. It was my hope that participants would include both teachers 
who had conducted home visits and teachers who had not conducted home visits, therefore resulting in a group discussion representing different points of view. Through engaging in this activity, participants may not have completely agreed on the benefits and detriments to home visitation, therefore as noted by Kitzinger (1994), participants had opportunities to question one another, disagree, misunderstand one another, or possibly try to persuade each other to see their point of view. I made sure to clearly state to the participants that the purpose of the focus group was not for the teachers who had conducted home visits to convince the teachers who hadn't done any home visits that they should. Rather, the intention was to explore the reasons teachers had or had not done home visits (i.e. what factors motivated them or what factors were barriers) and to better understand if the participants perceive a relationship between home visits and culturally responsive instruction.

Through the shared conversation, participants may have been moved to clarify their thinking or possibly re-think their point of view (Kitzinger), which therefore resulted in richer data pertaining to the topic of home visits than would be generated from individual interviews. I followed the focus group with a round of individual interviews also focusing on the topic of home visits. Using the data collected during the focus group session, I revisited and made adjustments to the interview questions for the third interview.

In order to ensure a quality conversation with multiple perspectives and diversity among opinions, the number of people participating in a focus group conversation should be large enough to merit a good discussion but not so large as to hinder the participation 
of all group members (“Guidelines for Conducting a Focus Group,” 2005). Johnson and Christensen (2008) suggest that the ideal size for a focus group is 6-12 participants while Morgan (1997) suggests 6-10 participants because "below 6, it may be difficult to sustain a discussion; above 10, it may be difficult to control one" (p.43). Although six people is typically the minimum number of participants recommended for conducting a focus group, I only had five participants because that number coincides with the number of cases I used for the study. Morgan suggests over-recruiting participants by $20 \%$, which ensures a large enough group if some participants discontinue their participation in the study or if certain participants are unable to attend the focus group, but based on the number of willing participants I had, I was not be able to use this safeguard. Therefore, I used Doodle, which is an electronic tool for easy scheduling, to plan a date/time that was convenient for all participants ensuring I had everyone in attendance. I was satisfied with the size of the focus group because Morgan also notes that larger groups can create difficulties for the researcher if participants are highly involved in the topic because members of a large group may break up into smaller conversations or people may begin to talking at once which both present challenges for audio recording and being able to hear each individual's comments or ideas.

Procedures for the focus group followed a protocol (Appendix G) and the conversation was video taped with a Flip Camera as well as audio recorded with the iTalk app on the iPhone device. All teachers attending the focus group first viewed a short video about culturally responsive instruction put forth by the Teaching Diverse Students Initiative. The viewing was followed by a 5-minute period of time during which 
participants were asked reflect on the video and to write down their immediate thoughts after having viewed the video. The notes written during this free write were collected and used as an additional source of data. Finally, I used discussion prompts and questions listed in the focus group protocol to begin a conversation about home visits. It was my intention to facilitate the conversation and observe participants rather than actively participating in the conversation. I did not offer my own personal ideas, thoughts, or reflections; rather, I entered the conversation only to offer another question or discussion prompt.

The audio files from the focus group conversation were transcribed verbatim for data analysis. Additionally, the video recording of the conversation was available in case I was unable to identify a particular speaker in the audio recording of the focus group session. When transcribing the conversation from the focus group session, I did not need to use the video footage in order to identify speakers.

\section{Artifacts From CARE Training}

During CARE training, teachers were asked to collect and record below the line information about their chosen focal students. Additionally, teachers were asked to create a series of culturally responsive lesson plans focused on objectives from the motivational framework for culturally relevant teaching (Appendix A). As part of the observation process, teachers were asked to write reflections both before and after the implementation of their culturally responsive lessons. It was my intention to use these articles in conjunction with the second round of interviews to assist participants in describing and discussing how they went about creating culturally responsive lessons. I thought that if 
participants had access to their notes and lessons it allow them to describe in greater detail the process of creating lessons geared towards meeting the needs of their culturally and linguistically diverse focal students. Unfortunately, only one participant brought any of these artifacts to the second interview, therefore most participants described their culturally responsive lessons from memory rather than using their documented lesson as a guide for explaining their planning process and the details of how the lesson was conducted. I received copies of CARE lesson plans from one other participant after the interview process had concluded.

I requested copies of these artifacts so that I could use them for further analysis when comparing data across cases with the idea that retaining hard copies would also allow me to look more comprehensively at patterns and trends in how lessons were created or perhaps to identify differences in how various participants undertook this assignment as part of the data analysis. I received copies of lessons from two out of the five participants, and because I didn't have copies of lesson plans from each participant, the written plans were not as significant a contribution to data collection as I had hoped.

\section{Field Notes and Research Log}

As I went through my research, I had a lot of my own reflections based on what I heard from participants. I kept a journal of my reflections and ideas that come about as a result of interacting and listening to participants during interviews and the focus group. I took time to write down reflections following most interviews in order to unpack my personal experience of the conversations. I believe writing down my own reflections directly following each interaction with participants allowed me to keep my own 
assumptions and interpretations from interfering with or introducing bias to my interpretation of the participant data. I also used this log as a way to further analyze the data when comparing across cases. Although I am in the role of the researcher and not a participant in the study, I still reflected on how participants' experiences compared with my own when evaluating the data.

The research log also contained my interview schedule, participant contact information, and notes on themes emerging from the cases in addition to my notes and reflections. The log served as a way to organize data and begin data analysis.

\section{Ethical Considerations}

Participants in the study were asked to share their ideas and reflect on their experiences. I do not think the study posed significant ethical threats of harm to participants given that teachers became participants by choosing to be involved in the study and they had the right to discontinue their participation in the study at any point.

It is feasible that participants may have had feelings of uncertainty or concern about discussing negative experiences, unfavorable opinions, or frustrations related to the process of CARE training and creating culturally responsive lesson plans within the context of a district that mandates the implementation of scripted curricula with fidelity. It was made clear to participants; however, that everything they shared as part of the study would not in any way be connected with their true identities.

Additionally, teachers may have experienced discomfort stemming from fear of exposing their ignorance or struggles with a peer. Fortunately, due to the Courageous Conversations protocols mentioned previously, CARE team members have practiced and 
become accustomed to "speaking their truth." It is understood among CARE participants that discussions remain free of judgment and that each CARE team member is entitled to have his or her own opinions about and interpretations of the experience. Again, participants were informed that everything they said would remain anonymous. Also, participants were encouraged to speak their truth as it would increase the likelihood that future professional development opportunities pertaining to culturally responsive instruction will address aspects of the process of creating culturally responsive lessons that were challenging for CARE team members.

\section{Timeline and Schedule}

Following a successful defense of the study proposal in early June, a Human Subjects application was submitted by mid-June 2012. A request to conduct the study within District CC was also submitted for review by the Director of Curriculum and Instruction. The application was reviewed by Portland State University quite quickly and upon approval of the Human Subjects application by Portland State University and permission from the district, I proceeded by inviting all elementary teachers who were part of their schools' CARE teams to participate in the study via district email.

Participant selection was completed by the end of June 2012 and I scheduled the first round of individual interviews to take place during July 2012. The second round of interviews took place in August of 2012. The focus group took place in late August and final interviews took place in October of 2012. Data analysis commenced in November once all transcription was completed and continued for approximately three months. 
I also had an alternative plan for data collection in the event that my anticipated timeline was not met. In this case, each stage of data collection would have been pushed back by approximately one month (Appendix H). My data collection timeline ended up being a combination of my two plans as I was not able to schedule the focus group until the very end of August and then waited until October to conduct the final round of interviews as I wanted to allow participants sufficient time to get settled into the new school year before taking their time for the final interview.

\section{Data Analysis}

The study was designed to contribute to the body of knowledge that currently reflects a separation between instruction based on the implementation of scripted curricula and instruction that is attuned to distinct needs, learning styles, and funds of knowledge of culturally and linguistically diverse students. An investigation of multiple cases was conducted with the intention of collecting extensive data that would allow me to describe in detail how teachers have experienced the process of a) trying to bridge the gap between implementing scripted curricula and creating culturally responsive lesson plans and b) trying to better acquaint themselves with the funds of knowledge of their culturally and linguistically diverse students. It is my hope that my research will inform the field of education in terms of the extent to which culturally responsive instruction might be used in conjunction with mandated scripted instruction as opposed to only in a constructivist context where teachers have more flexibility in designing their own lessons. 
Data analysis for the study followed Creswell's (2007) template for coding a case study (Figure 1), which provides an organizational structure and sequence for data analysis procedures. I began by transcribing the audio content from the interviews as well as the focus group conversation so that I could organize the data by cases and read the content thoroughly and repeatedly. I documented thoughts and memos in my research log and wrote notes and ideas for initial codes in the margins as I read the transcripts in order to capture and facilitate analytical thinking as well as to form a list of potential codes (Creswell; Yin, 2009).

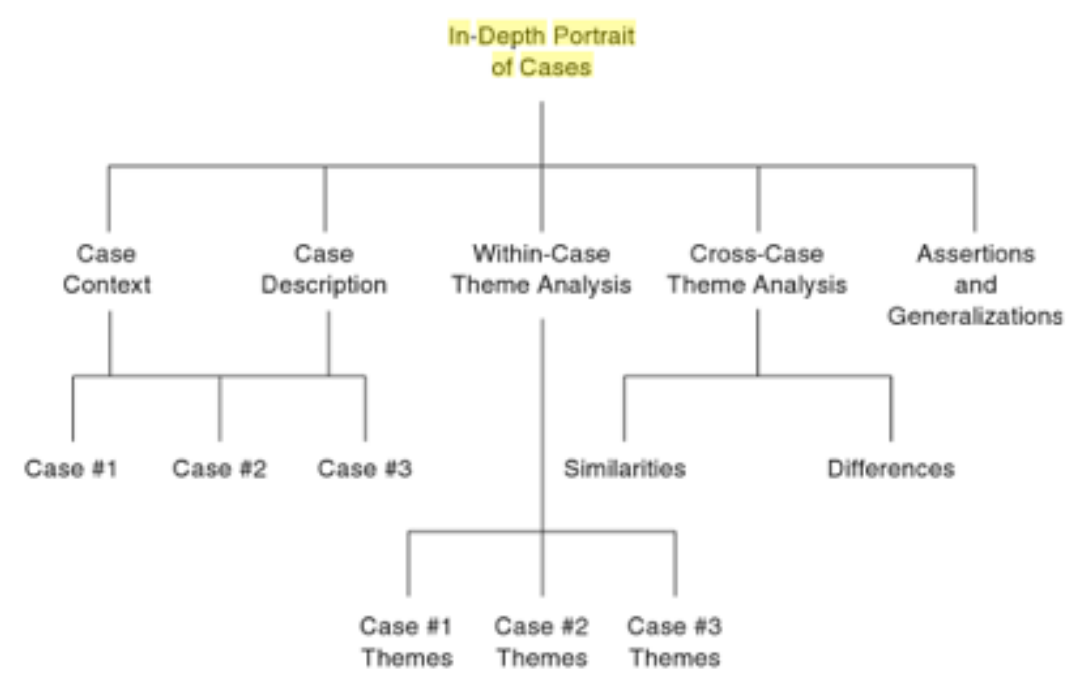

Figure 1. In-depth portrait of case study data analysis (Creswell, 2007).

In order to interpret the data in different ways, I conducted three phases of data analysis (Figure 2). I used both within-case and cross-case analysis to determine themes that emerged from each individual case as well as themes that emerged from the 
collective group of cases. The final phase of data analysis consisted of making assertions about the overall "lessons learned" (Lincoln \& Guba, 1985) from the case study.

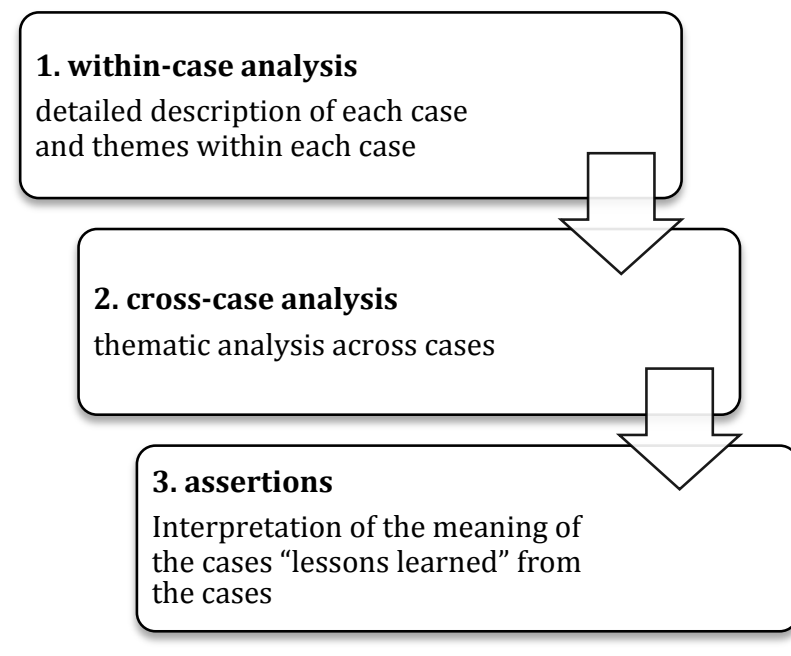

Figure 2. Phases of Data Analysis

\section{Phase One: Within-Case Analysis}

To complete the first phase of data analysis, I used the qualitative software program HyperRESEARCH primarily to assist with organization of data through coding. Saldaña (2009) describes a code as, "a word or short phrase that symbolically assigns a summative, salient, essence-capturing, and/or evocative attribute for a portion of language based or visual data" (what is a code?, paragraph 1). Despite this fairly straightforward definition of a code, Saldaña offers approximately thirty different types of coding methods with the indication that each qualitative study is unique and there is not a "best" way to code qualitative data. I reviewed each approach to coding in order to determine which would be most appropriate and beneficial for my study and decided that structural coding, descriptive coding, and values coding were each a good fit, and would compliment one another, to better organize my data in preparation for the next part of 
analysis. Per Saldaña's recommendation, I wanted to "mix and match" (Overview of First Cycle of Coding Methods, paragraph 1) several approaches to potentially bring more depth to my analysis. Table 5 provides a brief overview of the purpose for each cycle of coding. Each cycle will be described in more detail in its own subsequent section.

Table 5

Overview of Coding Strategies and Their Purpose

\begin{tabular}{lll}
\hline $\begin{array}{l}\text { Cycle 1 } \\
\text { Structural Coding }\end{array}$ & $\begin{array}{l}\text { Cycle 2 } \\
\text { Descriptive Coding }\end{array}$ & $\begin{array}{l}\text { Cycle 3 } \\
\text { Values Coding }\end{array}$ \\
\hline $\begin{array}{l}\text { Grand tour overview of } \\
\text { data }\end{array}$ & $\begin{array}{l}\text { Breaks down data and } \\
\text { categorizes segments }\end{array}$ & $\begin{array}{l}\text { Applies codes based on } \\
\text { participants' value, beliefs, } \\
\text { and attitudes }\end{array}$ \\
$\begin{array}{l}\text { Segments data according } \\
\text { different topics of inquiry }\end{array}$ & $\begin{array}{l}\text { according to the "essence" } \\
\text { of what was said }\end{array}$ & \\
\hline
\end{tabular}

Cycle 1: structural coding. Saldaña (2009) recommends employing structural coding to achieve a "grand tour" overview of the data. Saldaña references MacQueen, McLellan-Lemal, Bartholow, \& Milstein (2008) to describe structural coding as a question-based code that, "applies a content-based or conceptual phrase representing a topic of inquiry to a segment of data that relates to a specific research question used to frame the interview" (Saldaña, 2009, Structural Coding, Description). I created a distinct code for each of my five research questions, selecting a phrase of key words from each question to use as the code. I then proceeded to go through each interview and assigned codes to larger chunks of the text based on which of the five research questions that particular portion of text addressed. Given that I organized the interview questions for the three interviews around separate themes, or areas of interest (i.e. culturally responsive teaching, planning/implementing culturally responsive lessons, and home visits), it was 
fairly easy to go through the interview transcripts and assign the codes that reflected the topics of inquiry and my research questions. Structural coding did not play a big role in the second phase of data analysis, but it did serve the purpose in the first place of helping me to learn the HyperRESEARCH program as well as helping me better familiarize myself with my data. Using structural coding was a preliminary way to help me organize the data, but additional coding was necessary to further categorize the data by content and topics. I chose to follow structural coding with descriptive coding and values coding in order to further organize my data by determining the variety of topics that were brought up and talked about within those broad topics.

Cycle 2: descriptive coding. Saldaña (2009) notes that descriptive coding is also called topic coding because it essentially uses a short phrase to categorize or describe the basic topic of a particular passage or segment of text. Bearing this in mind, as I read through the transcripts line by line; I broke the data into chunks and assigned to those chunks a code representing the "essence" of what the participant was talking about in that sentence or phrase. As I went through the first few interviews, I created codes based on the main ideas and/or salient topics that were discussed by the participants. Where appropriate, I used a "word or short phrase from the actual language found in the qualitative data record" (InVivo CODING, Description, paragraph 2) to create codes, which is a strategy for coding called InVivo coding. Saldaña notes the importance of creating codes that represent the topics included in the data rather than abbreviations of the actual content. As I got further into this first phase of data analysis, I took time to reflect on whether a particular portion of data could truly be represented by a code that 
was already in the codebook or if it required the creation of a new code. By the time I was coding the final few interviews, most of the data could be coded with an existing code; however, there were a few instances when the participant spoke about something that did not fit into any of the existing codes. In those instances, I created new codes in order to ensure that the codes were fitting the data and not the other way around.

I viewed the coding process as a way to categorize the pieces of data into groups, so that I could then attend to the content of the data. My coding system allowed me to classify sections of data by topic so that I could later opt to view all of the data pertaining to certain topics. Focusing on smaller portions of data that were assigned to the same code allowed me to synthesize and analyze what participants said about the topic, how the various topics they spoke about fit together or did not, and what themes were apparent for individual cases as well as across all of the cases.

Cycle 3: values coding. My final cycle of coding consisted of values coding, which is the "application of codes on qualitative data that reflect a participant's values, attitudes, and beliefs, representing his or her perspectives or worldviews" (Saldaña, 2009, VALUES CODING, Description, paragraph 1). I chose to utilize values coding because I wanted to sift through the data with explicit attention to identifying statements or passages that reflect the participants' personal values, beliefs, and attitudes.

Saldaña (2009) differentiates and defines values, beliefs, and ideas for clarity; yet, states that, "Values coding does not necessarily have to code for all three or differentiate between them" (VALUES CODING, Applications, paragraph 2). When I conducted this round of coding, I did not feel it was necessary to code the data as specifically a value, 
belief, or attitude; rather, I coded any statement or portion of text that I considered any one of the three with the same code name. I also chose not to attach any further description to the code so that the data pertaining to values, beliefs, and attitudes would not be fractured into smaller categories based on the theme of each value, belief, or attitude. My intention was to look with a wider lens at all of the data pertaining to values, beliefs, and attitudes in order to synthesize how those values, beliefs, and attitudes play a part in the "story" of each case.

Utilizing values coding seemed particularly relevant for one participant, who expressed herself with strong convictions regarding what it meant to her to be a "professional" and what "her job" as an educator entailed. I wanted the ability to view the data relating to values, beliefs, and attitudes separately in order to consider how each participants' values, beliefs, and attitudes related, if at all, to the themes that emerged from descriptive coding cycle as well as to "paint a better picture" of each participant. In order to best describe each participant in the individual case narratives, I felt that looking for, and being aware of their values, beliefs, and attitudes was pertinent. Conducting values coding was easier with some cases than others because certain participants were more vocal and direct with making statements about what they believe in or what they think is very important. Because each case is unique, I conducted values coding for all five cases; yet, I did not "force" the data into this category if it didn't seem to fit.

Re-organization of data. My goal for the second part of data analysis was to better understand possible categories and themes by manipulating and exploring all of the categories that I established during coding. As a visual learner, I decided that I needed to 
move away from the technical process of coding in order "see" the data more clearly. I began by writing my five research questions as well as all of the codes I created on a small index cards. I then placed each index card containing a code underneath the research question to which it best applied. This was primarily a way for me to visually see all of the categories as well as how the categories related to one another and how the categories related to the overarching research questions of the study. The arrangement I created (pictured below) remained on the floor of my office to reference as I proceeded with further analysis.

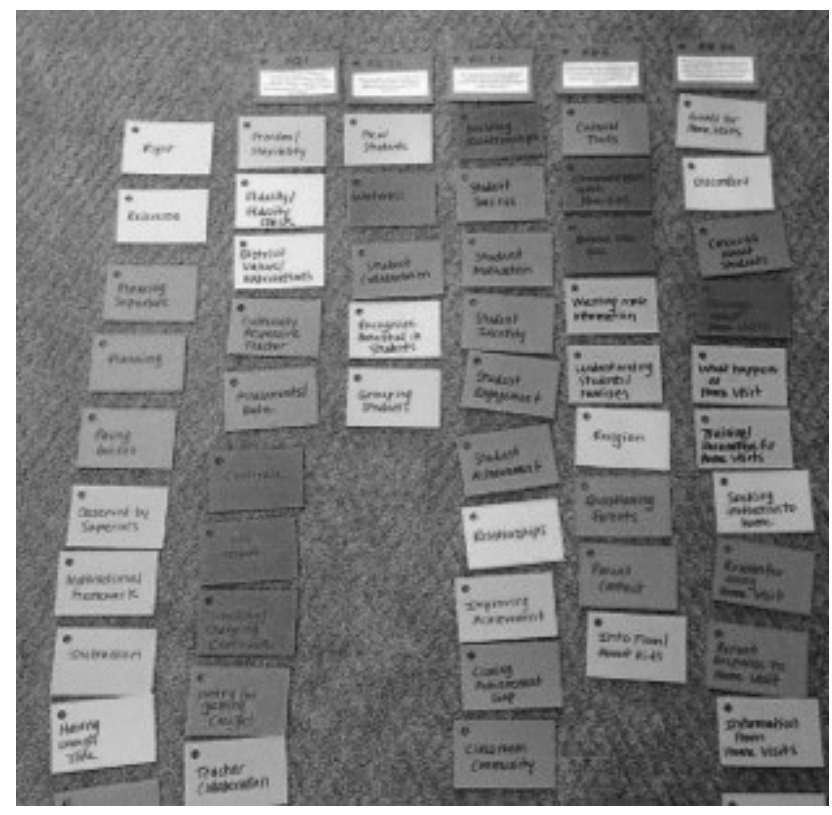

Figure 3. Visual Organization of Research Questions and Codes

Following the creation of a visual that represents the relationship between the codes and research questions, I used the frequency report tool within HyperRESEARCH to view the total number of times each code was used for each case. When viewing the frequency report for each case, I looked to see which codes were used with the most 
frequency for each case (the modes). In the majority of the cases, there were 2-3 codes that were assigned to data more frequently than the remaining codes. I used the codes that appeared most frequently for each case as a center point to create different mind-maps that tied together the various "details" of data associated with each particular category. I created the mind-maps using a program called Popplet, which is a tool that allows users to visualize ideas through the use of graphic organizers by connecting ideas in textboxes called "popples." After placing the "main" code in the center, I used the filter tool within HyperRESEARCH to view all the data related to that particular code. I re-read the data pertaining to the main code and added additional popples to the mind-map containing direct quotes from the interview transcripts and detailed data from that particular category of data. Within each mind-map, I divided the data into sub-categories to better organize the data within the theme and better understand the larger picture of that data representing that theme.

To make sure I didn't leave out any data that was pertinent to the main theme, I used another function of the filter tool in HyperRESEARCH to filter the remaining codes by building criteria. Building criteria involved selecting several codes in order to view the data pertaining to all of the included codes at once. I used the original visual map as a guide to decide which other codes to investigate so I could sift through additional data that could potentially overlap the main theme and add depth. I tried different combinations of codes as criteria for filtering the data and read the accompanying data until I felt confident that I had included all the ideas and thoughts pertinent to the main theme. 
I printed the mind-maps for each case and used those to create a written narrative describing the participant and the main themes that emerged from her data. I referred to the mind-maps to weave together the specific details from the data and quotes from the participant in order to tell her story making sure to include the content from each popple. I also referred back to the artifacts participants wrote at the focus group session as well as the notes in my research log pertaining to each case to ensure that I was incorporating all of the types of data that I had collected. Each case narrative includes a detailed description of the participant and various aspects of her teaching. The narrative is meant to provide the reader with a story that allows him or her to have a clear picture of the poignant and significant aspects of that participant's story. The case narrative concludes with my synthesis of the main themes that emerged from coding and re-organizing the data.

\section{Phase Two: Cross-Case Analysis}

Cross-case analysis is a strategy for data analysis that involves examining themes across cases and to determine themes that are common to all cases (Creswell, 2007; Yin, 2003). Conducting cross-case analysis allowed me to see the themes and/or differences between participants' reports of their experiences. During this stage of data analysis I focused more on fracturing the data from particular cases and "re-sorting it into categories that facilitate comparison between things in the same category and that aid in the development of theoretical concepts" (Maxwell, 2005, p. 96). At this point in the data analysis, I was looking to establish what similarities and differences existed between the 
cases, so I began to take a broader look at the data by zooming out to consider data from all five participants at once.

I began my cross-case analysis by using the frequency report tool within HyperRESEARCH once again; however, for this phase of data analysis I did not filter the cases so that I could view the frequency of each code across all of the data. I viewed the code frequencies in descending order in order to determine which codes were used most often. Using the frequency report, I made a list of which codes were used most often and originally chose the frequency of 29 as my cutoff point. Consequently, any code that was used 29 times or more was included on my "master list." The table below shows the various codes and corresponding frequencies. 
Table 6

Frequencies of Most Repeated Codes

\begin{tabular}{ll}
\hline Code & Frequency \\
\hline Tweaking/Changing Curricula & 102 \\
Feelings about Home Visits & 80 \\
Info about/from Kids & 70 \\
Curricula & 67 \\
Teacher Collaboration & 40 \\
Parent Contact & 56 \\
Communication with Parents & 42 \\
Below the Line & 42 \\
Building Relationship & 41 \\
CARE Lesson & 41 \\
Information from Home Visits & 39 \\
Training/Parameters for Home Visits & 35 \\
Reasons for Home Visits & 32 \\
\hline
\end{tabular}

After compiling the master list of the codes, I considered which of those codes were related to one another so that I could combine some of the codes into broader categories that would then encompass the specific codes. The table below illustrates how I combined and, in most cases, re-named the various codes on the master list. When doing this, I referred back to my research questions in order to stay focused on the 
inquiries driving the study. Looking back to the research questions helped me think about which different codes could be combined to paint a complete picture in terms of answering those questions.

Table 7

Master List of Reoccurring Codes

\begin{tabular}{ll}
\hline Broader Categories & Codes Included \\
\hline Home Visits & Feelings about Home Visits \\
& Reasons for Home Visits \\
& Information from Home Visits \\
& Training/Parameters for Home \\
& Visits \\
& Parent Contact \\
& Communication with Families \\
Parent Contact & Curricula \\
Interplay between Scripted Curricula and & Tweaking/Changing Curricula \\
Culturally Responsive Instruction & CARE Lesson \\
& Info from/about kids \\
Better Understanding Focal Students & Building Relationships \\
& Student Engagement \\
& Below the Line \\
& Teacher Collaboration \\
&
\end{tabular}

Once I had combined codes and created broader categories, I was able to utilize the filter tools within HyperRESEARCH to view the data relating to all of the codes in any particular broad category at once. At that point I used a variety of strategies to further organize the data coming from all five cases including creating tables, making lists, and re-reading larger portions of the interview transcripts without focusing on the coding to get a "big-picture" sense of what the data was saying. For each of the broad categories, I 
wanted to determine if the participants expressed common ideas, which indicated a consensus in perspectives and consequently a theme to be asserted. I also wanted to determine around what points the participants did not agree because the divergences could also inform emerging themes for the study.

\section{Phase Three: Assertions}

The final phase of data analysis took place in the form of a last layer of processing what participants shared both individually, and collectively, in order to reflect and draw conclusions about what it all means and the "lessons learned" (Lincoln \& Guba, 1985). For clarity and organizational purposes, I have included a discussion section about each case following the case narratives in Chapter 4. I also included a discussion section for each of the major themes that emerged via cross-case analysis after the section on each theme. The final assertions for the overall implications for the study are included in chapter 5 . 


\section{CHAPTER IV}

\section{RESULTS}

In this chapter, I will describe the major themes that emerged within each case as well as the themes that were meaningful across the different cases. For each case I will provide some information on that participant's teaching experience, why she decided to become part of her school's CARE team, as well as some brief information about her focal students and how she went about selecting them. I will present a narrative to showcase each participant's unique experiences followed by a discussion of the themes that were reflected in her story. Then, I present and examine four themes that emerged from my analysis of the collective group of cases, which represent the common ground among the five cases. Table 8 provides a glimpse at the pertinent themes for each of the five cases as well as the themes that applied to all of the cases collectively. 
Table 8

Summary of Within-Case and Cross-Case Themes

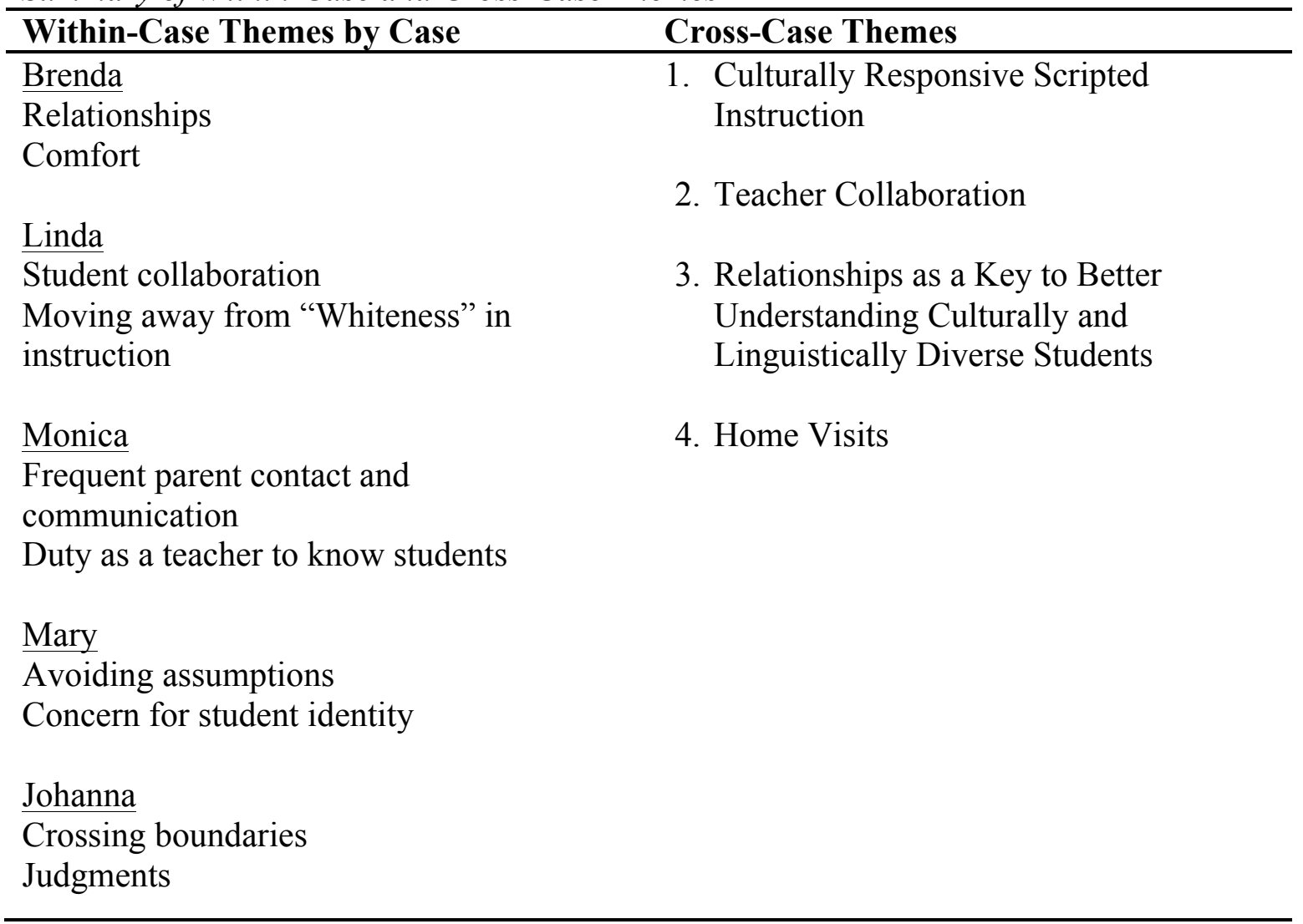

\section{Context for the Cases}

Each of the cases is a teacher who attended a series of CARE trainings within District CC throughout the 2011-2012 school year. The training consisted of 4 full-day training sessions as well as interim assignments that teachers conducted at their school sites with their fellow CARE team members. Assignments included selecting 3-5 students of color to serve as "focal students," collecting below the line information (both defined below) about those focal students in order to get to know them better, taking notes on what engagement looked like for each of those students, and finally creating a series of 
culturally responsive, "CARE," lessons geared towards better engaging the selected focal students. CARE participants worked with a Motivational Framework for Culturally Relevant Teaching (Appendix A) and were asked to select some of the criteria from the framework (i.e. rigor, relevance, relationships, and realness), when planning their CARE lessons.

Reading instruction was the primary area of interest in the study because of the extent to which reading instruction is highly scripted; yet, CARE lessons were in the areas of Math, Social Studies, Language Arts, and English Language Development in addition to Reading. Although some CARE lessons did utilize district-adopted curricula that are not scripted (i.e. the Social Studies curriculum that Mary could not recall the name of), each participant created several CARE lessons and the majority of CARE lessons were in Reading or Language Arts utilizing scripted programs. Table 9 includes the various district-adopted curricula used by teachers during CARE lessons.

Table 9

District-Adopted Curricula Used in CARE Lessons

\begin{tabular}{ll}
\hline Subject & District-Adopted Curricula \\
\hline Reading (Elementary) & $\begin{array}{l}\text { Success For All FastTrack Phonics } \\
\text { Macmillan/McGraw-Hill Treasures }\end{array}$ \\
Language Arts (Middle) & Voyager Learning LANGUAGE! \\
Math & Houghton Mifflin Math Expressions \\
English Language Development & $\begin{array}{l}\text { Susana Dutro Systematic English Language } \\
\text { Development } \\
\text { Ballard \& Tighe Carousel of IDEAS }\end{array}$ \\
Social Studies & Not identified by name \\
\hline
\end{tabular}


CARE teachers participated in a collaborative effort to observe and give feedback to their fellow CARE team members in order to help the instructing teacher determine the effectiveness of her CARE lessons for her focal students. Because the participants often refer to terms or words used in CARE training, I think it is important to acknowledge which terms that the participants used came directly from the CARE training. Each of the terms, or expressions, below was explained, discussed, and used in the context of CARE training. Although no formal definitions were provided to CARE participants, understanding of these terms and expressions was built through reading articles and engaging in discussions. Within the CARE community, these terms were used as common language; therefore I did not place emphasis on asking participants to define specifically what they meant when they used these them during the interview process. For the purpose of clarity, I have provided a brief definition for each term, based on my personal understanding of the concept.

- Below the line information: Information about students and their families that pertains to their particular lives as opposed to generalizations about their cultural group. Obtaining below the line information refers to understanding aspects of students' lives that may not be overtly apparent, similar to the section of an iceberg that is hidden below the water.

- Focal Students: 3-5 students of color chosen to be the recipients of teachers' extra attention and consideration when planning and implementing instruction

- Whiteness: Behaviors, attitudes, mindsets, and actions that exemplify White privilege 
- Decentering Whiteness: Specifically considering how one's Whiteness influences their thinking or behavior and attempting to put that aside in order to incorporate other perspectives into classroom curriculum and instructional practices.

As previously stated focal students fit within the category of culturally and linguistically diverse students, as used in my research questions. I also conducted the study with the belief that for all intents and purposes the term below the line information is consistent with the concept of funds of knowledge and therefore the terms were used synonymously. To ensure consistency between the specific wording in the data collected and my findings, I will use the terms focal students and below the line information from here onward.

Given that each individual participant reported experiencing the CARE process in difference ways, it is not surprising that unique themes emerged for each case narrative. The themes highlighted in the case narratives represent aspects of each participant's experience that stood out most to that individual.

\section{Case 1: Brenda}

Brenda is a half-day Kindergarten teacher with four years of teaching experience in the same school where she currently teaches. She decided to become part of her school's CARE team because her team was very interested in the topic of culturally responsive instruction. Brenda selected an African American male student with behavior problems, one Hispanic student who was low-performing, and one Marshallese student who was low-performing to be her focal students. She identified unfamiliarity with 
students' backgrounds, low academic achievement, and behavior problems as reasons for choosing those particular students as her focal students.

Over the course of the three interviews, Brenda spoke frequently about her relationships with her students and how the process of creating CARE lessons and receiving feedback from colleagues who observed her helped her to realize there was a need to work on building relationships in her classroom. She attributed the lack of relationships to an overt focus on academics in Kindergarten and said, "Sometimes I think I forget about how important relationships are" as well as "You can realize all of a sudden that you don't know them and they don't know you." Brenda first opted to focus her CARE lessons on rigor, which the Motivational Framework for Culturally Relevant Teaching describes as "challenge and engagement," because she wanted to please her superiors and she felt there was a school wide emphasis on teaching with rigor. Yet, feedback from her colleagues indicated that some of her focal students did not appear to be comfortable when activities called for peer-to-peer interaction. Her focal students were observed not sharing their thoughts or answers and standing on the edge of the carpet during a mixer game that reviewed letter names. Brenda shared that she realized she couldn't focus on rigor without first helping her students to build relationships with one another. She needed her students to feel comfortable enough speak in class and share their ideas with other children. She recognized the need to specifically teach her students how to build relationships with one another and that it was important "not to just build them once but continue the community thing." Brenda realized her students needed time to grow comfortable enough to share and stated, "They have to trust you." In order to 
build stronger relationships with her students and among her students, Brenda intentionally made efforts to make personal connections, particularly with her focal students. She began checking in with them as a way to show them that she cared and made efforts to find out what they were interested in.

Brenda realized that when planning her instruction, she couldn't make assumptions about shared experiences and she couldn't just try "what she thought worked.” Rather, she knew that she could better reach her students by understanding them, which meant looking for what kinds of things they enjoyed and what they needed to feel comfortable. Brenda reported that she collected most of the below the line information about her students through observation and by having conversations with them. However, she spoke about two barriers to learning more about her students: 1) she was not able to get as much information from her student who was lower on the language level scale and 2) she felt that simply observing students led her to make assumptions. When I questioned Brenda about why she thought that observing students led her to make assumptions, she explained that when she was observing students in her classroom she questioned whether she was gathering information that was true about the students or she was just assuming something was true based on what she saw. For example, she said one of her focal students seemed very social because when he talked, he seemed very comfortable. She thought he would do really well with the interactive mixer game she planned, but in that whole group interactive atmosphere, he turned very introverted and he looked uncomfortable. She shared that even though the conversations were short, she felt she got more "real" information from talking with her students, compared to 
observing them. Additionally, Brenda mentioned that her preferred times to observe students was during unscripted times such as first thing in the morning or during playtime, but those opportunities were rare. Brenda reflected that collecting below the line information changed her relationship with her students. For instance, regarding her focal student whom she described as having behavioral problems, Brenda stated, "it made me want to work with him more."

Brenda was also able to gather more information about her students from their parents and their siblings, but she commented that in some cases she wished she had more contact with parents beyond the chance encounters at school and phone calls. She made efforts to include more questions about families during her spring conferences and was comfortable asking specific questions about things they like to do at home and if they went to preschool, but explained that she didn't want to ask "probing" questions that would be intrusive such as inquiring why a student did not have particular background knowledge about something that was talked about in class.

In terms of classroom activities and instruction, after getting to know her focal students better and trying to figure out who they would connect with and what commonalities they had with other students, Brenda began pairing students with a particular partner because she reconsidered what she was asking students to do in front of the whole class rather than on an individual basis. She found that students were learning better in one-on-one relationship building activities as opposed to the mixer type activity she had done previously. She noticed her focal students were "catching on to things more quickly" and "their knowledge came out more quickly." 
At several points Brenda talked about wanting more information about students and their families, particularly when her students had behavior issues or attention issues because she felt like she could be more understanding and have more empathy if she could better understand the situation they were coming from. She mentioned wanting to better understand "their world." When speaking about home visits as a potential way to learn more about students and their families, Brenda indicated that she felt limited by only ever hearing about students' homes as opposed to experiencing them first hand and drawing her own conclusions. Brenda said, "I think the value is really amazing of a home visit [but] I think it's still scary to approach." Brenda did indicate that she would be willing to do a home visit; yet, she had many concerns about doing home visits. She mentioned not having enough time, not having any training, feeling timid, feeling hesitant due to information she already knew about a particular family, fearing families wouldn't receive a home visit well, fearing that she would offend a family, and not knowing exactly how to broach the topic of home visits with families.

Brenda shared a story about an experience she had in which a family that receives a food backpack from school didn't have transportation to retrieve the bag themselves when their child was absent on the day the bags were sent home. She called the family offering to bring it to their home and felt that the parents were apprehensive about her coming and did not want her to. Brenda ended up offering to drop the food backpack on the family's porch and the family agreed, so she did visit their home without interacting with the family or entering their home. Even though she didn't communicate with her student or the family during her visit, Brenda still thought she was able to get pertinent 
information from her visit. She reflected that she had made some assumptions about the type of apartment complex the student lived in, believing that it would be "rundown, packed with people, and not super well kept up." When she visited, she encountered a complex with duplex houses with little backyards. She described the location as “intimate," a "very close neighbor community," and "not what I expected." Although she felt she got valuable information from the drive-by visit, as a result of feeling as though she was not welcome to actually visit the student's home, Brenda feared that if she tried to initiate home visits with other families, they, too, would respond with a closed door. She worried that parents would think the intent of her visit was to check up on them and made several references to not feeling comfortable with going unless she knew the parents were receptive and open to the idea.

Brenda said she would be willing to try home visits indicating she would feel more comfortable knowing that the parents were comfortable with it and having a specific reason for visiting or a more concrete objective like when she had to drop the bag off. In that situation, she knew the family needed the food, so she didn't question her personal comfort level as much. She also expressed interest in attending a training about how to conduct home visits because she would want a protocol to follow and coaching on how to make sure she didn't offend anyone because she wouldn't want to do anything "to hurt the relationship you're building with those parents." Brenda thought that parents might be more comfortable with the idea of home visits if it was a district initiative or if home visits were done in replacement of conferences. 


\section{Themes In Brenda's Case}

I interpreted Brenda's lesson plans, her reflections about her CARE lessons, and her ideas about home visits to consist of themes of the relationships and comfort. Brenda worked hard to build relationships with her students and their families as well as to facilitate stronger relationships among her students. She believed that by getting to know her students, she would be able to connect with them better in order to help them feel more comfortable in the classroom. Brenda recognized that her students needed to trust her and to feel comfortable in her classroom in order to truly demonstrate their knowledge and participate in classroom activities. Brenda's reflections indicated that during the CARE process, she experienced a big shift in her thinking about what she should focus on in her CARE lessons. She started out focusing on rigor because she felt it was emphasized by the district; yet, she quickly realized that she needed to take a step back from rigor and develop relationships with her students in order to have them engaged in academically rigorous activities. She took ownership of how to best apply what she learned in CARE with her particular students, even when that meant shifting her focus away from what she felt the district was pushing for.

The idea of comfort was also important to Brenda with regard to home visits because she wanted to make sure she could go about initiating and conducting home visits in a way that made parents feel comfortable. Additionally, she wanted to ensure her own comfort by learning more about how to conduct home visits so she would be more prepared. Brenda's discomfort around asking parents if she could visit their homes stemmed from her personal experience with her first attempt at home visitation. Brenda 
feared that if she attempted to inquire about visiting other families, they too, would be cautious and hesitant. Therefore, Brenda became very concerned about how to approach home visits so that she would know how to broach the subject with parents in a way that would not make them uncomfortable or suspicious about her intentions. Brenda was interested in learning about the process of home visitation as well as learning strategies to ensure she would not unintentionally do something to offend families. Brenda thought that she would feel more comfortable talking with parents about home visits if she was more knowledgeable and prepared for the process.

\section{Case 2: Linda}

Linda is an educator with 21 years of experience primarily in Kindergarten, which she currently teaches and has been teaching at the same school for the past 11 years. She has taught at schools with a variety of socioeconomic and diverse settings, going from a school with a very high Hispanic population and very low socioeconomic level to a school with a very high socioeconomic level and a primarily white student population out in the country. She described the school where she currently works as "mid between the very high and the very low." In addition to teaching Kindergarten, Linda taught first grade for several years and was a Title I Reading Teacher for one year.

Linda decided to become part of her school's CARE team because she was asked by the principal and she is "always open to doing something that will benefit the kids" and "learning things that will help students become more successful." The focal students Linda chose were three students who she saw as struggling academically in the class. When explaining why she chose them to be her focal students she said, "They were going 
to need the best instruction that I could give them" and "I was going to give them more time than the other students." Her three focal students were all Latina females. She shared that she had six students of color in her class, but that the other students of color were either "on" or "ahead" academically so she wasn't worried about them. She also took classroom performance into consideration when choosing which students would be her focal students and explained that the students she picked "really weren't engaged in learning." Linda talked about what she meant by engagement in saying that she wants her students to be at the carpet or at their table and absorbing information. She said that prior to CARE, her focal students were "shoe tiers" and "shoe players." They also "played with each other's hair" and "were more interested in each other than learning."

When Linda talked about trying to better understand her focal students, she shared that she had some difficulty collecting below the line information directly from her students because, “young kids don't necessarily share about their culture, they share more of their age appropriate interests." She indicated that the types of things her students would tell her about did not match the kind of information she was seeking. When she inquired about what her focal students do at home, they shared things such as playing with Barbies, playing tag, and riding their bikes. Linda expressed that she felt her students were telling her what they thought she wanted to hear when she was really looking to find out something about them that she didn't already know or to learn more about their cultures. She also indicated that it was difficult to collect below the line information from kids who speak a native language other than English because those 
students may be more comfortable sharing in their native language, which she did not understand.

Rather than discontinuing her efforts to collect below the line information, Linda enlisted the help of a Spanish-speaking instructional assistant who supported the ELL students in her classroom. She asked the assistant to pull her group of focal students into the hallway and have a discussion with them in Spanish. Linda was not able to be part of the discussion; yet, she indicated that she got a lot of below the line information about her focal students by having them speak in their native language in a small group setting to an adult who also speaks that language. She thought that perhaps the girls were able to make a better connection with a teacher who looked more similar to them and who could speak with them in their native language. Linda said the girls "really kind of fed off of each other and told more about their families and their homes than just an interview." And, the information the girls shared during this group discussion really helped Linda to better understand the dynamics she saw between them in her classroom because she learned how interconnected the girls' families are.

Although Linda was able to get a fair amount of below the line information with the help of her instructional assistant, she indicated that when she began to plan her CARE lessons, she still relied on "generalisms on cultures rather than specific below the line information because it just was really hard to get." She used general information about different cultures she got from the CARE trainings pertaining to how students or children in those cultures learn and work to make changes to her teaching. Over the course of the interviews, Linda repeatedly talked about recognizing the need to move 
away from the "Whiteness" in her teaching. She said that looking at her CARE students, their cultures, and their backgrounds "awakened" her to see culture and background as reasons why students learn differently and to think about how to address that in her instruction so that all of her students would be actively engaged in learning. She spoke about moving away from the Whiteness in her instruction and her desire to develop a classroom in which students who experience social groups differently than the way she was using them in class could have access to learning opportunities that fostered more communication and collaboration. Linda expanded on what she meant by moving away from the Whiteness in saying, "so it's just not learning the White way you know sitting in rows and repeat and repeat." She also shared that she thinks the White philosophy in education is that students should work independently and that students should not share their work because they need to do all their own learning. She categorized the aforementioned description as a very White way of thinking, which is the kind of thinking that she was trying to move away from by introducing more opportunities for her students to collaborate with one another.

Linda recognized how important it is for students who come from collectivist cultures to experience opportunities to work together in school and said that student collaboration was a "big aha moment" in her CARE work. She said that most of her CARE lessons were designed with collectivism and students working together in mind because "learning socially and learning or working with peers was the piece they understood." Linda said, "they were really comfortable with that social and talking and 
moving" and she attributed that to their cultural background. During her CARE lessons, Linda had her students work together in whole group activities as well as in partnerships. Linda described one of her activities as a mixer with letters and sounds where "instead of just using flashcards, which is how the scripted program does it," she handed out cards to the kids and they had to say the sound on their card, listen to their partner, and then switch and go. She said, "Rather than memorizing, they were teaching their partner and then receiving instruction and then switching." Linda explained that the children were given the answer in advance if they needed it and then repeating it to someone else, which was another layer of practice. She said her students loved the activity and that rather "turning off," her focal students would listen to what their partners said. Although this type of collaborative activity was successful for most students in her class, she explained that it "allowed an out" for a student who might be "walking around looking like she was doing something when she really wasn't," which is the behavior she saw from one of her focal students. So, Linda also utilized student partnerships as a way for students to work together and collaborate.

Linda said she assigned students to pairs and was very mindful of who she assigned each child to work with. For her focal student who got somewhat lost in the mixer type activity, working with a partner elicited a higher level of engagement. Linda thought her lessons utilizing partners were very successful in terms of getting that particular student engaged and she said, "just by having that connection my student was more involved." She thought her focal student seemed more confident and comfortable and that helped her to be more successful. 
When reflecting on her collaborative activities, Linda was able to offer some before and after comparisons of her focal students' engagement in the classrooms. She indicated that before she introduced collaborative activities, one of her focal students would not even come to the carpet to join the class. Instead she would walk around the room or turn her back in the other direction and Linda could tell, "She didn't want to be there." The other two focal students were the "shoe tiers" who Linda said were not engaged in learning at all and preferred to focus on each other or the laces on their shoes, rather than instruction. She indicated that once she started teaching her CARE lessons, two of her focal students became active participants and she could see that they were more engaged and noticed they had more confidence and enjoyment in the activities. Linda's third focal student did not become engaged as quickly; however, through the paired activities and Linda's continued efforts to get her engaged like the other students, she was "right in there working with the other kids and the other kids working with her."

\section{Theme's In Linda's Case}

Analysis of Linda's discussion of her CARE lessons and classroom activities reflected the themes of engaging students through the use of student collaboration and also moving away from the "Whiteness" in her teaching. When interviewing Linda, I was very struck by her commitment to change aspects of her instruction that likely have been part of her pedagogy for many years in order to meet the needs of her current students.

She seemed very devoted to doing everything in her power to ensure the success of all of her students, which required her to reflect on her teaching practices and make adjustments so that students from collectivist cultures could experience more 
opportunities for collaboration and communication with peers. She intentionally made efforts to engage students who were disengaged from the "White" ways of teaching that required them to sit and listen as well as complete work independently. Linda transformed her classroom into a community of classmates who were capable of learning from one another, teaching one another, and navigating learning tasks side by side while offering support to one another. Linda's ability to reflect on her practice contributed to her use of instructional strategies that she considered to be more culturally responsive and more likely to result in success for students who were not flourishing when she asking students to complete work independently.

\section{Case 3: Monica}

Monica is currently an English Language Development (ELD) teacher who works with students in Kindergarten through Fifth Grade. She began teaching in 1990, but "retired" to stay home with her four children after teaching Sixth Grade for four years. She returned to teaching in 2006 "fully focused" on English as a Second Language (ESL) and ELD. Monica teaches ELD in a pullout model, so she removes ELD students from their regular classrooms to work with them in a small group for thirty minutes daily. She is the only ELD teacher at her school, so she works with all of the students who are acquiring English. Her groups are created based on the students' grade levels and language levels. She explained that per protocol, children with no more that two different language levels could be placed together in a group for ELD instruction. She had also extended beyond her role as ELD teacher to serve as a Family Liaison position. Monica shared that the Family Liaison position involved a lot of work including setting up 
counseling, medical, and dental appointments for families as well as organizing the food backpack program and getting parents hooked up with ESL classes.

Monica indicated that she became part of her school's CARE team because she is "very into social justice." She also had additional experience serving on other teams that were formed as part of the Pacific Educational Group professional development series. She became part of her school's Equity Team in 2008 and was then invited to be part of the District's Equity Team. Monica said the focal students she chose were some of her Fourth Grade English Language Learner (ELL) students who were "heading towards Special Ed. referrals." She indicated that she wanted to see if the extra attention, her involvement with their families, and/or the cultural responsiveness that were built into the CARE process would "ease them away from that Special Ed. referral." Monica shared that both of her focal students were children of single parents and that they had a "lot of foundational holes" in their education. She also mentioned that their families often discuss moving back to Mexico.

Over the course of the interviews, Monica spoke in depth about her communication with families and the different ways she contacts parents. Monica expressed very passionately that "families need to be contacted" and indicated that she communicates with parents frequently because she feels "it's her job" to do so. She said, "building relationships with parents is part of the focal student thing" and so she spends a lot of time making efforts to do just that. Monica makes calls to families daily and keeps a call $\log$ where she writes down the gist of the conversation and the information she gathers while speaking to parents. If she cannot reach parents, she will leave a message 
on the machine indicating that she needs to speak with them and wants to see them. She also provides parents with her personal cell phone number so they can reach her if they need to and she indicated that parents "totally respect boundaries." Monica listed six reasons why she calls parents were: a) concern for students with behavioral or academic difficulties, b) to build relationships with parents, c) to collect prenatal data and information on students' infancy, d) to collect information for a review of family history if she is beginning the referral process, e) to try and get families involved in the Hispanic Advisory Committee and f) on behalf of her colleagues who need her to contact Spanishspeaking parents because she is a fluent speaker of Spanish. She noted that she always starts out by speaking in Spanish, if appropriate, and she tries to "come off real casual" because her facial expressions cannot be seen through the phone. She indicated that she tries not to use formal academic speech patterns; rather, she positions the parent in the role of expert about his or her own child and initiates a collaborative relationship between herself and the parents in an effort to help the student. Monica said she often talks to the student's mom and she tries to come off "real sister like" and is willing to share information about herself. She told me a story about calling the mother of a young girl about whom she was concerned to ask if she could invite the student to lunch and get takeout food. When she asked if the student liked rice, the mom said of course she likes rice, we eat rice every night. Monica shared that she eats rice every night too and they had a laugh about the habit shared between their two cultures. If she is calling to offer support to parents, such as the food program provided by the school, she again said she 
tries to sound "really casual" and lets them know what is being offered, but that it's "no biggie."

Monica shared that she has encountered parents who were fearful and/or cynical towards contact from her because her school has experienced a lot of turnover with ELD teachers and because parents were concerned about deportation. However, Monica talked about how the students' excitement for her class made parents most trusting because they attributed their children's' success to her teaching. She also tries to counter parents' fears by explaining to them that she is interested in giving their children more attention and wants to spend more time with the students to help them be successful.

When I questioned Monica about communicating with parents who don't speak English, she said that most of her students' parents do speak English, but she did mention a few strategies she uses for parents who do not. She indicated that she uses Google Translate to translate any documents that need to go home to homes where parents don't speak English. She also created a phone tree of parents who are bilingual in other languages and are willing to offer support to parents who share their language but don't speak English. Monica was able to create this tree as part of her work when she held the Family Liaison position a few years back when she was teaching at a different school. She said that by looking through a database containing student information (eSIS), she was able to identify families speaking languages other than English and she knew if the students were not on her ELD caseload, the parents and students likely spoke fluent English. She was able to contact parents who were fluent in other languages and request their help translating for families who needed support understanding communication 
from the school. She said she always made sure that all parents involved understood that the conversations were confidential and that parents were comfortable with their children being discussed. She also indicated that the principal had approved the phone tree idea. In addition to expressing the importance of contacting and communicating with families, Monica was vocal about the importance of knowing, and relating to, her students. When describing her idea of a culturally responsive teacher, Monica said, "I think it's definitely getting to know the little faces that are sitting there with you, getting to know their likes and their dislikes. It takes extra time. It goes beyond the workday. It goes beyond the contract. But, it's the mark of a better teacher." She also said a culturally responsive teacher "goes above and beyond the call of duty" and when I asked her if she considers herself a culturally responsive teacher she replied yes. Monica said that she feels she is a culturally responsive teacher because she makes the effort to know the families and makes efforts to put her students' interests into her lessons. She elaborated on her view by saying that she is a professional, her students are her clientele, and her job is to "sell them education." She declared, "I have to know how they're gonna buy it."

In order to know her students better Monica says she hands out little information cards at the beginning of each year to collect information about their favorite foods and other things they like. She also relies on conversations with the students and said they are talkative and "they'll tell me anything." She also began listening to her students' favorite radio stations as a way to familiarize herself with the music they liked and then integrated popular songs and music video clips into her lessons, but changed the lyrics to incorporate math concepts. Monica said that her students were "thrilled that the stuff that 
was part of their normal regular life was also academic and they loved coming to class." She indicated that she tried to stay "keyed in" on different aspects of their cultures and "keyed in" on things that would naturally motivate anyone in order to get those kids closer to her and more motivated. She shared a story of one student who adored Star Wars and who was so excited and engaged when he found out that she also loved Star Wars enough to name her son after one of the characters. She said her student was highly motivated to do extra work in order to get a page from her Star Wars coloring book to take home and that she didn't think she would have done something like that before the "below the line intensity stuff of CARE."

When she reflected on how her CARE lessons impacted her focal students, Monica said she thought the lessons gave them more confidence, made them feel more successful, and made them like school just a tiny bit more. Monica said:

I do believe what is said about children really being able to measure how important they are in the eyes of their educator and if they really feel like they're a burden, they will not perform. They will choose not to perform. Or, they will rise to the level that is expected of them.

Monica also talked about situations when she felt she wished she knew more about particular students and what efforts she made to get to know them better. One strategy she used was to do some research on students' cultural groups in order to have more broad background knowledge on particular culture's history and behaviors. For one particular island culture, she learned that they are a collectivist society and that oral history is very important in terms of passing down information. She also found out that they were very concerned about protecting their land and were interested in 
environmental issues. Monica said that her research plus information from particular authors and researchers she trusts was a good source of information. However, Monica also expressed the importance of getting information that is specific to the student as opposed to the general information she obtained about the cultural groups to which students belong. Monica spoke extensively about the benefits of home visits in terms of getting a lot of information about students and their families. She said:

A million points of input come from that home visit and then by getting all that below the line information you can turn that into teaching that responds to their culture and whether it's another country's culture or another facet of American culture, you can teach towards that.

Monica says that she has never invited herself to a student's home, but she often seeks an invitation. When she interacts with her students she hopes that through conversation and their similarities, she will get an invitation to visit some homes. She made it clear however, that you won't get invited if you don't have some kind of relationship with the parents or the family beforehand. She referred back to the importance of taking the time to make a few phone calls in order to make a relationship as a precursor to seeking an invitation to visit a family at home. Monica discussed one benefit of home visits being that she could see right away what they have or don't have and what they're needing. She mentioned making efforts to help families by getting assigned a particular student as her Christmas kid in order to help provide items that were needed as well as having one of her students stay after school so that he could have a quiet place to do homework because there wasn't a place in his home for him to get work 
done. Monica said that her relationship changes with students and she is more understanding when she can see what their life is like at home.

\section{Themes In Monica's Case}

Monica was a very interesting teacher to get to know. She is clearly very passionate about teaching and dedicated to knowing both her students and their families well enough to provide students with the very best educational experience possible. Two themes were prominent in her work as an educator, which were importance of frequent contact and communication with parents and her belief that getting to know her students as individuals is a core element of her duties as a teacher. Monica spent time daily reaching out to parents to ensure that they were connected and involved with their students schooling. She connected with parents for a variety of reasons, always having their best interests in mind. Monica explained her actions as a sense of duty, indicating that it was "her job" to contact families, but her stories and explanations about her communications with families were not weighted by a sense of obligation. Rather, her commitment to her students and their families seemed driven by her high level of knowledge about working with ELD students and what it takes to ensure student success. She clearly demonstrated that the extra effort and time put towards building relationships was well spent.

Interestingly, Monica was the only teacher to identify herself as a culturally responsive teacher compared to the other four participants who indicated that becoming culturally responsive is a progression and they were still working on it. Monica created lessons specifically for the CARE process; yet, it was evident that her beliefs about 
connecting with families and the actions she took to know her students and their families on a deeper level were part of her repertoire prior to CARE. Monica stood out as an educator who understands culturally responsive practices and who would be an excellent resource for training teachers about how to conduct home visits. She provided a lot of examples with specific details about how she communicates with families and how she expresses interest in what students do outside of school as a way to get an invitation to visit their home rather than just asking if she can visit. Her strategies for connecting with students involved a lot of time and effort; yet, she was confident in the value of her efforts.

\section{Case 4: Mary}

Mary is a Fifth Grade teacher with six years of teaching experience. She currently works at a school with a very high socioeconomic status, which she mentioned is quite different from the inner city environment where she completed her student teaching and her first year of teaching in Sixth Grade. Mary decided to become part of her school's CARE team because she had previously participated in Sheltered Instruction Observation Protocol (SIOP) training as well as Beyond Diversity training. She said that she thought the CARE trainings would "go hand in hand" with the training experiences she already had because of the focus on teaching in culturally responsive ways for students of color. She shared that she thinks cultural competency is important and having grown up in California, she is used to a lot of diversity. Yet, a particular activity she participated in during the Beyond Diversity training made her think more about the "lack of color and diversity" in Oregon, which pushed her to desire a better understanding of how to make 
diversity more relevant in the classroom. When Mary decided on her focal students, she chose students of color who were underachieving in her class. She said that her school does not have a high population of students of color, but does have a lot of Asian students. She chose a Chinese male who she described as "underperforming" and "behaviorally immature," a Palestinian male who "underachieves" and "has some reading difficulties," and a Chinese female who was adopted by White parents.

When Mary reflected on what she learned about culturally responsive teaching, she talked in detail about the importance of getting to know where students are coming from and not making any assumptions based on, "what they look like, their name, or even meeting their parents." She said she realized she shouldn't assume that her students share a common background with her even if they are White like she is. She said, "There are times when I have to be careful about decentering my own Whiteness." Mary said that it's important to understand different cultures; yet, "you can't judge a kid by that because everyone's so different." She reiterated that even within a class where many students are White, she doesn't want to assume that students will automatically understand something just because it's part of White culture. Mary used the example of a story that is included in the reading curriculum she teaches that is about a spelling bee. To Mary, a spelling bee is representative of competition in school, which she said is, "kind of an American thing." Yet, Mary recognized that she could not read the story with her students just assuming that they knew what a spelling bee was. So, she did a lot of pre-reading questioning about what it's like to be in a contest and talked with her class about different contests and different challenges. Mary also said that it's important not to, "single kids 
out as a representative of their culture." When one of her Muslim students was fasting during the celebration of Eid, she said the other kids wanted to know more about it. She was torn because she didn't want her Muslim student to, "stand up there and be the representative about Eid," but his classmates were curious. Mary decided to talk with the student and his mom to make sure it was okay for him to share some information with the class, but she said she felt like she had to "walk a fine line."

In addition to recognizing that she shouldn't make assumptions about her students, Mary also talked about assumptions in a different context. She said that in the texts and novels she reads with her students, often there is an assumption that the main character is White simply because the story takes place in America and nothing within the text indicates otherwise. Mary noted that many books do have White males as the lead characters and this led her to consider whether her classroom atmosphere and the book choices she makes allow all students to feel like their cultures are represented. She wondered if all students are able to "find themselves in the material." She thought, "Do kids see people like them in the literature they read?" Mary mentioned that she was excited about the half Black main character in the Rick Riordan series because his books are modern and not historical fiction. She also said she makes efforts to choose stories with female main characters.

Mary reflected that part of being a culturally responsive teacher is making sure to have discussions about topics in curriculum texts that relate to different cultures or different perspectives. She talked about how she will now address topics in her instruction that she may have felt uncomfortable talking about in the past. Mary shared 
about an incident that another Fifth Grade teacher at her school experienced, in which a student's race became the topic of discussion. Mary explained that when the Fifth Grade students were asked to do an activity requiring them to be American colonists, one African American girl in her teammate's class did not want to make herself Black when she had to design a little person and make a doll of herself. Mary acknowledged that it was difficult for that student because she was the only Black girl in the Fifth Grade, so there weren't any other kids sharing her experience. In addition to collaborating with an ELL teacher about this issue, her colleague had a private talk with the student in order to let her know that it was okay for her character to look like her and it was also okay if she wanted to make her character look like a White colonist. Mary used this as an example of a conversation that was uncomfortable, but at the same time something the teacher was willing to talk through. When I asked Mary if she thinks Fifth Graders are equipped to talk about issues like this one that come up in class, her response was that she thinks it depends a lot on the students' parents and how the students' feel about themselves. She mentioned that focal student who is Chinese and was adopted by American parents was very proud of her culture and excited about it because her parents have helped her be proud of her roots. Yet, other kids were definitely aware of their differences and they may have felt self-conscious even if other kids didn't treat them differently. Mary shared that, "Ten is a strange age" and that kids can get picked on for a variety of reasons that don't necessarily have to do with their color. So, she thinks it's important to help kids understand that whether or not they choose to talk about their issues, school is an appropriate place to talk about it. 
Mary said that she feels like she is still learning how to be a culturally responsive teacher but that something she has noticed about herself is that she now speaks up when conversations with other people rub her the wrong way. When another teacher was talking in the staff room about an OPB documentary about Native Americans, and made a generalizing statement about how "they are all this way," Mary spoke up to challenge what was said. Mary said it's hard to speak up without being too defensive or seeming like she is attacking someone; yet, she "couldn't just sit there."

\section{Themes In Mary's Case}

Conversations with Mary as well as her written lesson plans revealed the themes of avoiding assumptions, concern for student identity, and addressing topics even if they are uncomfortable. Although Mary says she is still learning how to be culturally responsive, she has made great strides in recognizing the importance of getting to know her students and understanding that knowledge of a student's cultural background does not automatically translate into specific knowledge about that student. Mary's recognition that she cannot make assumptions about her students leads her to be very cognizant of her students' personal identities in terms of a) getting to know her students as individuals and b) ensuring that her students feel as though their identities are represented in her classroom, her instruction, and in the books/stories she chooses. Mary's comments about speaking up to her colleague who was making generalizing comments about Native Americans and about how she makes sure to stop and talk about culturally related themes in the stories she read with her students are both illustrative of shifts in her teaching and within herself that reflect culturally responsiveness. She indicated that prior to CARE 
training, she probably would not have said anything to her colleague and she would not necessarily stop to talk about uncomfortable topics that arose in the curricula she teaches because both of those scenarios can be uncomfortable.

Mary brought up an important idea when she talked about not having a single child represent his or her entire cultural group, which is equally as important as not using generalizations about a particular culture to define an individual. Mary had a clear understanding that information she learned about a particular child did not translate to knowledge about that child's broader cultural group and she was cognizant that cultivating discussions with her students about race, culture, and their personal identities needed to occur in a way that prevented assumptions or stereotypical thinking. It was evident that Mary valued knowing her students as individuals and wanted all her students to know that they are valued and important in her classroom.

\section{Case 5: Johanna}

Johanna is an educator with four years of experience teaching Special Education at the middle school level to students in sixth, seventh, and eighth grades. She is currently working as a Special Education teacher at the elementary school level. Johanna became part of her school's CARE team because she was asked by her principal who wanted someone to bring the Special Education perspective to the team. She said she was a bit worried about how much time the commitment was going to take, but she thought "it was good" to be part of the team. Johanna selected three students to be her focal students because "they were Hispanic," they "had low skills" and "were also very needy students in many ways." 
When Johanna talked about how she went about learning about her focal students, she shared that during activity time in her classroom she would sit around with different groups and "just talk to them and listen to them." Johanna said that when her students felt comfortable with her, they gave her a lot of information, but at times, her students told her "more than she wanted to know." Often, students would come to her with complaints about other teachers or they would share about their "difficult" home lives, which she referred as "intense negative drama." Johanna also said she heard "a lot about girl drama." When I asked Johanna if she was able to get any information from her focal students that informed her about their cultural backgrounds, she said "yes," and shared that they talked to her about their friendships, how they interact with their families, and how their families relate to school. Johanna said she found it interesting to learn about their different holidays and, "how they went about doing it with their family and their extended family." She also brought up that at times she "didn't necessarily agree" with some of the things her focal students said occurred in their homes. Johanna shared that she tried to "compartmentalize" that the things she heard were happening at home and just loved the students "for who they are." She said she recognized that there were some things she couldn't change; yet she did try to provide her students with information and to help them see different options that were available to them. Johanna tried to give them another option "without being judgmental towards their family." She wanted to "give them a different viewpoint" and used her own family as an example. She wanted to let her know how she could support them. Johanna shared that her students began to seek her out to talk to her and she often utilized the little landing outside her 
portable classroom as a space where students could talk with her privately. She described herself as a "go to person" whom students would come talk to and she felt very good that her students were comfortable to share their problems with her and felt like her relationship with her students changed as a result of her listening and their conversations.

One particular student shared information with Johanna that made her concerned. She followed up with the school counselor and that student's parents in order to clarify and confirm what she had heard. Although she was concerned, she said she was empathetic towards the parents and tried to figure out what the school could do to help, including what services the family might need that were available through the school. Johanna said that she felt as though her focal student shared some "very intense" information with her because she needed to "download" everything so she could be in a place to learn. However, because of the content of what this student shared, the details of which are not appropriate to share here, Johanna was in the position to question whether or not to "take action." When I asked her to clarify what she meant by "take action," she said she called Child Protective Services.

Although she was pleased with her ability to build trust with her students so that they felt comfortable talking with her and sharing their problems and concerns, Johanna was worried that conducting home visits would result in boundaries being crossed that could negatively impact their understanding of her role as a teacher. In addition to thinking that middle school students would not be excited about having their teacher come to their homes, she was concerned that visiting homes would result in a familiarity between her and her students that would blur the lines between "being a professional and 
wanting to be friends." She acknowledged that home visits offer an opportunity to gather information about students via "feelings and impressions" as opposed to researching particular cultures which might result in stereotypical information. At the same time, she felt home visits are a more appropriate practice for elementary school.

As a Special Education teacher, Johanna had annual meetings with parents and had to collect information about the students on her caseload as part of the process of preparing the students' Individual Education Programs (IEPs). She said sometimes the conversations with parents were "a little uncomfortable," for her, but she thought it was probably easier for her to ask certain questions than a regular classroom teacher because she has to ask them frequently as part of her role as a Special Education teacher. She indicated that asking questions pertaining to whether or not parents could afford certain things was more difficult. Johanna explained that when she questions parents, she can indicate to them that it's a way that she is looking for information and collecting data.

When I questioned Johanna about her thoughts on whether or not parents' culture influences their expectations of Special Education, she prefaced her response by saying "Not to stereotype, but..." and shared that "Hispanic families expect behavior to be taken care of at school" and that "school problems are school problems." She also said that, "Some of the families don't understand the importance of homework." Johanna listed several examples of what might be occurring in a student's home that would inhibit their abilities to find time to focus on homework such as taking care of siblings, sharing a household with other families, and not even having a bed to sleep on. Yet, she said parents still expect their children to get A's and B's in school. She mentioned that 
"schools in Mexico are different and the parents don't really interact," so she would try to draw them in and would tell them, "This is your part. This is what you need to do" in order to get the parents more involved in their children's education.

\section{Themes in Johanna's Case}

Two themes that emerged from the analysis of Johanna's stories and experiences were the crossing of boundaries and judgments. The complexity of the issues in Johanna's experience warranted more unpacking on my part; therefore the discussion of Johanna's themes is a bit longer than the discussions following other cases. The issue of boundaries was evident in Johanna's story about having to report information about her student to authorities. Although she wanted to be a good listening ear for her students and wanted to be available to them in terms of helping with their problems, because teachers are mandatory reporters, it came to a point when she had to "take action" due to the information that was shared with her. The intention of collecting below the line information about students is to get to know them in a more intimate way in order to have information about them that informs curriculum and instruction. Yet, the information that is learned from and about students can be sad, upsetting, or confusing. In the case of one of Johanna's focal students, Johanna became privy to information that put her in a tough position. The nature of the information shared was intimate to the extent that Johanna's legal obligation as a mandatory reporter overrode her role as confidant to the student. Johanna also specifically addressed the idea of crossing boundaries when discussing the prospect of home visits. She was cautious about the idea of visiting students in their homes because she was concerned about becoming too familiar with students in a way 
that would negatively impact their relationship and their understanding of her as a professional.

Additionally, the theme of judgment emerged throughout Johanna's discussions with me. Johanna explicitly expressed her desire to refrain from judging her students; yet, apparently she experienced some tension around this issue. Although she didn't want to be judgmental, she did state that she was opposed to certain behaviors that took place in one student's home. What is significant, however, is that she wrestled with how to carry on with her students in a positive way, when she knew information about their life at home that she didn't agree with that she described as "perfectly acceptable in their family."

Also, when speaking about her Hispanic students and their families, Johanna did make some statements that generalized behaviors of particular families as traits associated with their cultural group. She did this when she indicated that Hispanic families expect behavior to be taken care of at school and don't understand the importance of homework. Similar to how she expressed her intentions not to judge her students, she prefaced her statements about Hispanic families with "not to stereotype, but..." which I believe is a red flag to signal that the following statement is likely going to reflect a stereotype or generalization about a particular group. I believe Johanna had very good intentions with her students and did connect with them in ways that allowed them better engage with her instruction. Yet, I also believe that Johanna's understanding of culture and the depth of her reflection about her own beliefs, and perhaps biases, would benefit from further unpacking and consideration. At times, she demonstrated 
deficit thinking, which places the responsibility of students' failures on their homes and family (Nieto \& Bode, 2008). Deficit thinking was evident when Johanna claimed that Hispanic families don't understand the importance of homework because rather than evaluating the nature of her homework assignments, how she communicated with Hispanic families, or trying to take into consideration situations that might have prevented students from completing their homework, she placed blame on the parents by saying they don't value homework. Johanna also repeatedly mentioned students' holidays and celebrations as evidence of their culture, which reflects a limited understanding of the complexity of culture. To Johanna's credit, the disequilibrium that was evident between how she intended to act and some of her statements represents an opportunity for her to further develop her identity as a culturally responsive educator.

\section{Cross-Case Themes}

As previously noted, the within-case analysis included a narrative detailing each participant's unique experience, which highlighted the particular themes that stood out within each case. Yet, it is also essential to consider the common ground among all of the cases. By conducting cross-case data analysis, I was able to explore the attitudes, values, beliefs, behaviors, ideas, and/or actions that participants shared. The themes that will be discussed in this section represent commonalities among the collective group; yet, they do not necessarily overlap the themes that emerged from the individual cases because I intentionally reserved the discussion of the data pertaining to cross-case themes for this section. Participants did not always agree entirely; however, several themes and consistencies became apparent as I continued to explore and analyze the data. 
Participants shared beliefs, ideas, experiences, and perceptions about the significance of relationships as a key to better understanding focal students, culturally responsive scripted instruction teacher collaboration, and home visits. Although parent contact was included in the prominent topics of discussion among participants, Monica was the only participant who described her methods and strategies for parent contact in detail. Therefore, Monica's discussion of parent contact was included in her case narrative.

\section{Culturally Responsive Implementation of Scripted Curricula}

When participants created their CARE lessons, they each had to negotiate how to best use the curricula that was provided by the district while at the same time attending to the needs of their focal student(s). Data from participants indicated that implementing scripted curricula with fidelity was important in terms of meeting district expectations. Several participants made reference to "fidelity checks" and Linda shared that since the adoption of the reading program the district currently uses, teachers are no longer able "to do our own things." Linda also shared that as a member of the math committee, she knows that teachers are not expected to be on the exact same page everyday; yet, she stated that there is "kind of an underlying message that you don't deviate from the curriculum and you can create new things but they have to be aligned with this curriculum." Mary indicated that her understanding of the district's message was that teachers need to teach to the standards but it is up to them as professionals to decide on the best way to do so. She said that her principal is very supportive of different things teachers have done, but she pointed out that "we're not taking anything away from the Treasures program but we're enhancing it." 
In sharing about their CARE lessons and their thoughts on how to teach in a culturally responsive way while implementing a scripted curriculum as the primary resource for instruction, participants shared several common perceptions. When responding to my inquiry about what elements of a scripted curriculum are nonnegotiable in terms of adhering to fidelity, participants agreed that the lesson objectives, vocabulary, and skills should not be altered. They indicated that it is important to ensure that students are working on the same outcome that the lesson indicates and teachers should keep with the "heart" of the lesson, but perhaps utilize more "variety." Monica said she, "wouldn't cut much, just teach it differently." Participants had many ideas for how to alter lessons to better meet the needs of their focal students in ways that would not impact those key elements of the scripted program such a having students work together instead of working independently and creating interactive activities that offered built in support to students who needed help with the content. They agreed that the pacing and delivery components of instruction are flexible and can be altered to suit the needs of particular groups or particular students. Participants indicated that pacing guides don't always work and that sometimes it is necessary to modify the pacing guide in order to fit the calendar, to take more time when needed, or to organize or sequence the lessons differently. Monica shared that she doesn't always do everything in the program and Johanna shared that she often takes "spontaneous deviations based on what comes up" during her instruction. Mary felt it was important to spend more time to "understand cultural pieces" and sometimes she would shorten or modify other parts of lessons. 
Monica also said she would "cut stupid stuff to make time for background building and cultural stuff."

Additionally, participants talked about ways in which they change the "delivery" of the lessons in order to make their instruction more engaging for their focal students. Examples of how participants changed the delivery of content were using additional books, using hands on activities instead of paper, providing examples other than the ones written in the curriculum, using comparison words for vocabulary, using different materials or manipulatives, using additional teacher created outside resources, making lessons more interactive, using the curriculum materials in different ways than indicated in the lessons, using music and math raps to teach concepts, bringing in real life examples, using games instead of worksheets, using games to teach or practice vocabulary words, and adding novels. Participants felt that it was important to try different strategies in order to make instruction "relevant to kids." Their intentions were to make instruction more "appealing" to their focal students and to help those kids "connect" to the content and instruction by "adding to the lessons" and "bringing in extra resources to make lesson more exciting" and "significant." Monica also indicated that classroom management could be used to teach lessons differently than they are indicated in a scripted curriculum. She did not provide any specific examples; however, several participants talked about utilizing more cooperative learning as a way to honor collectivist cultures, which could be an example of using pairs of students or groups instead of having students work independently. Additionally, a scripted program might indicate that students should be grouped by skill levels; yet, a teacher could use different 
criteria for grouping students based on her knowledge of their social skills, cultural communication patterns, or personality traits.

Discussion. When discussing how they created culturally responsive lessons to go along with the district's adopted curricula, teachers mostly reflected the attitude that they value what a scripted program has to offer. None of the teachers exemplified the rebel teacher as described by Eisenbach (2012) who used entirely self-created materials and did not use the scripted program at all. Rather, each of the participants exemplified the negotiator by demonstrating the ability to infuse their own ideas and beliefs into a scripted curricula (Eisenbach). None of the participants indicated that using culturally responsive instructional practices in conjunction with a scripted program was not feasible. Participants' CARE lessons did not always go as planned or elicit the results that were intended, but most participants agreed that their focal students became more engaged as a result of the instructional changes they made. Participants demonstrated that they understood their students' needs and preferences more deeply by altering their instruction in ways that made focal students more engaged and more excited about learning.

When addressing participants' culturally responsive implementation of scripted curricula, it is necessary to return to the earlier exploration of culturally responsive instruction in order to discuss which components of culturally responsive instruction (Gay, 2010) were evident from participants' descriptions of their CARE lessons during which they were still making efforts to adhere to the integrity of scripted curricula. In describing their CARE lessons and efforts to better engage their focal students, 
participants talked about activities and instructional strategies that reflected each of Gay's descriptors. However, participants did not discuss activities or instructional strategies that represent ethnic and cultural diversity in curriculum content to the same extent that they provided examples of attending to culture and communication in the classroom, culturally responsive caring, and/or cultural congruity in teaching and learning.

Participants' efforts to better engage their focal students relied more heavily on trying to build connections with their students and getting to know them better than on integrating below the line information about their cultural or ethnic affiliations into the instruction. Culturally responsive caring was particularly evident in participants' relationships with their focal students, which will be discussed in a subsequent section. The use of games and activities that required students to work collaboratively are examples that reflect participants' attention to cultural communication as well as cultural congruity in teaching and learning. Mary and Brenda used games for practicing letter recognition, number recognition and vocabulary. Both Linda and Brenda also used cooperative learning with their students based on their knowledge of collectivist cultures. Participants took time to get to know their focal students in order to become aware of how they learn, what types of activities they enjoy, and what types of learning situations help foster their understanding and communication of curricular content. Attending to learning styles is reflective of Gay's component of cultural contiguity in teaching and learning. Participants also altered aspects of their instruction marked by Whiteness such as when and how students speak up, how many student voices respond at once, and what 
role students have during instruction (listening passively versus being actively engaged), which reflect Gay's component of cultural communication in the classroom.

In creating this study, I was most interested in Gay's (2010) final component of culturally responsive instruction, which is ethnic and cultural diversity in curriculum content. I was particularly interested in the ways in which teachers learn about and incorporate students' funds of knowledge into their scripted instruction; yet, this final component was not well exemplified in participants' reports. Most of what participants shared regarding the incorporation of below the line information into instruction related to students' likes, preferences, and interests in order to facilitate better engagement and make students more interested in learning. For example, Monica listened to the radio station her students liked so that she could turn popular songs into math raps. She also utilized Disney Princesses and Star Wars items to motivate students. Efforts were made to incorporate students' perspectives such as when Mary asked students to tell about their families' histories of coming to America or when Johanna realized that her students were not quite understanding the concept of a vacation and then related the lesson content to how her students would often travel to Mexico. Yet, attending to the experiences and perspectives of culturally and linguistically diverse students only represents part of ensuring that curricular content is both ethnically and culturally diverse. The histories, heritages, and contributions of students' ethnic and cultural groups were not explicitly incorporated into participants' CARE lessons. A discussion of my emerging understanding of the differences between below the line information and funds of knowledge is included in the following chapter. 


\section{Relationships as a Key to Better Understanding Focal Students}

One of the main objectives for CARE participants was getting to know their focal students better by collecting below the line information and particularly focusing on what they look like when they are engaged in learning and what kinds of instructional tasks result in them being engaged. Participants used a variety of strategies for collecting below the line information including talking with students' parents, observing students in various school settings, talking with students in both structured and non-structured conversations, arranging for students to have conversations with instructional assistants in their native language, and using students' narrative writing pieces as a source of information. Although participants did focus on all four categories from the Motivational Framework (relationships, relevance, rigor, and realness) in their CARE lessons, they talked about the importance of relationships more than they talked about any of the other three aspects within the framework. Their focus on relationships relates to Gays' (2010) cultural caring in the classroom. Participants indicated that the relationship piece was very significant to their CARE work and this belief was evident in the inclusion of many aims from the Motivational Framework's section on relationships in their CARE lessons as well as their descriptions of the outcome for their focal students. Some examples of points from the Motivational Framework that were addressed in participants' lesson plans as well as in their explanation of their lessons were a) focal students talk with their partners in small group work; b) focal students have opportunities to respond to the lessons by writing or speaking; c) teacher demonstrates that she cares about Focal students; d) focal students and teacher have opportunities to learn about each other; and 
e) focal students and teachers have opportunities to learn about each other's unique backgrounds.

Participants talked about "checking in" with their focal students more often in order to make "personal connections" and learn about what their students like to do outside of school and what their interest are. Several participants talked about making concerted efforts to interact with and observe their focal students. Linda asked an instructional assistant to talk to her focal students in Spanish and the information gleaned from sharing in their native language gave her something different to talk to her focal students about. She said it gave her an "opening to talk to the kids about what they might have done at home" and that resulted in her learning even more about her students because she could inquire about topics specific to their lives and their families. Monica shared many examples of how she built relationships with her students including using parents as a resource for information, inviting a student to stay after school in her classroom to do his homework because he didn't have a quiet space at home, inviting a student to have lunch with her, and using her students' interests as a way to motivate them and make them excited about learning such as using the tunes of songs they liked for math raps. Several teachers agreed that it was important to focus on building relationships with students before focusing on other aspects of the motivational framework such a rigor. Brenda shared that she realized that she couldn't properly address rigor until she worked on building relationships with her students and on helping her students build relationships with one another. Once her students gained trust and confidence, they were more willing to participate in academic tasks and demonstrate their 
knowledge. Several participants indicated that they began utilizing more partner activities and tasks where students had to collaborate with one another in order to help students connect with each another. Participants used what they learned about their focal students to pair them up with other students with whom they could work well.

Participants agreed upon the importance of obtaining below the line information about particular students rather then relying entirely on generalized cultural traits to better understand their focal students; however, participants differed in their satisfaction about what kind of information they could get by talking directly to their students. The participants who taught upper elementary and middle school students reported that their students gave them a lot of information about their lives both through conversation as well as through their writing. The participants who taught Kindergarten, however, shared that it was difficult to get a lot of information from talking with their focal students, particularly focal students who were acquiring English. Linda said she felt like her students would tell her things they thought she wanted to hear such as about how they rode their bikes or went to the park over the weekend. She said she was not getting the kind of information that she was looking for until they were given an opportunity to talk about their lives in their native language with another native speaker.

Discussion. Although all four categories of the Motivational Framework are equally significant, participants' consensus in placing emphasis on relationships, which reflects Gay's (2010) culturally responsive caring, marries well with their ideas about how to successfully implement scripted curricula while also attending to culturally responsive instruction. Participants indicated that the actions they took to build 
relationship and implement collaborative student activities benefitted their focal students; yet, they were still confident that they were implementing the scripted program with fidelity because they didn't alter the main objectives, vocabulary, or skills included in the various programs. One participant described her instruction as “doing what we're supposed to be doing and doing it with thoughtfulness." Recognition that not all students communicate in the same way and concern for students' comfort level led participants to evaluate their teaching using a CARE-oriented lens and make adjustments accordingly. Participants' choices to alter the pacing, delivery of lessons, and means by which students were asked to demonstrate their understanding/skills are reflective of how participants were in tune with their students and adjusted instruction achieve better engagement form their focal students in particular.

Although participants did collect below the line information about their students, they did not share a lot of examples of specific below the line information that was used in conjunction with the content area subject matter content that they were teaching which would have provided examples of Gay's (2010) component of ethnic and cultural diversity in curriculum content. Rather, the below the line information they referenced related more to what they learned about students lives, home situations, families, interests, and/or personalities that then led to changes in their instructional strategies in content delivery instead of curriculum content. Below the line information helped participants to feel as though they better understood their focal students' perspectives and allowed them to empathize better with their students points of view, which resulted in positive outcomes for their relationships with focal students and evidence of culturally 
responsive caring. Connecting with students more often and taking interest in their lives by asking questions helped participants obtain below the line information. Obtaining below the line information then helped teachers reflect on their instruction and how those particular students would receive it,

\section{Teacher Collaboration}

Participants prepared and taught several different CARE lessons during the 20112012 school year and they also participated in a group observation cycle during one of those lessons. During the group observation cycle, each participant had an opportunity to be observed by the other members of her school's CARE team as well as the CARE facilitator in order to get feedback about one of her CARE lessons and the lesson's impact on her focal students. Participants also observed their fellow CARE team members' lessons in order to provide their teammates with feedback. Most participants shared that the group observation cycle was a positive experience that provided them with feedback about their culturally responsive instruction and helped them to better understand what their focal students were doing during the lesson. For example, Brenda pointed out that her CARE team members were able to notice behaviors from her focal student of which she was not aware and the group observation was very helpful in terms of her understanding which of the four R's she needed to focus on with her lessons. She said her CARE team noticed her focal student's facial expression during the mixer activity and she realized through their objective descriptions of his behavior that what they saw when they focused on his behavior was not the same as what she thought she 
saw. She said, "they could see that where I couldn't" and their observations were "completely eye opening."

Monica shared that her CARE team members gave her good ideas for how to extend the lesson she did and they also gave her an idea about "how to do partner selection better." She said working with her CARE team members was beneficial and she appreciated the opportunity to reflect on her lesson with them when it was over. She also mentioned that at times it was difficult to work the other adults on her CARE team because one member of her CARE team didn't follow through with the expectations like providing a written copy of her lesson to her team members prior to the observation. That individual then denied having done or said things in a particular way when those aspects of her lesson were discussed during the debriefing session. Therefore, Monica indicated that, "you have to have somebody who's really understanding what they're doing and they're really working with you on that." Overall, she still felt the group observations were beneficial because the feedback she got from her CARE team member was helpful.

Although most participants felt the group observation cycles were a positive contribution to their CARE experience, some indicated that conducting group observations doesn't seem like a feasible practice to use on a regular basis because of the extent of planning it required. Mary shared that her CARE team was able to carry out the group observation cycle by utilizing three student teachers to help cover classrooms. She also indicated that time was a factor and that the observation cycle was rushed because her team was tight for time due to the time period during which the observations were 
scheduled. Another participant felt that it would hard to do a group observation cycle again because "It takes people out of their classrooms." She felt like her colleagues were the most valuable observers and she thought it was not only informative, but also fun having them visit her classroom. She said she thinks it is "definitely doable," but it requires planning and having the support of someone who is available to cover classrooms when teachers are observing other teachers.

Discussion. In my experience, collaboration among teachers is crucial for understanding, interpreting, and putting into practice the knowledge that is acquired through professional development and training. The procedure of observing other teachers while they instruct students doubles as a learning opportunity as well as an opportunity to provide valuable feedback to colleagues about nuances in their classroom environments they may not be able to attend to in the midst of teaching. This practice is particularly valuable in the context of culturally responsive instruction and CARE work because teachers are interested in the facial expressions, emotions, and behaviors of their focal students, which may not be apparent to them as they teach an entire group of students. Unfortunately, when teachers leave their own classrooms to observe colleagues, it creates some logistical and scheduling concerns. A teacher cannot leave his or her own classroom unattended in order to observe a colleague; therefore, arrangements for coverage need to be made. Observations can be brief; yet, the benefits for the teacher being observed need to be carefully balanced with the time the teacher who is observing is not instructing his or her own students. With schedules that are already tight, and insufficient budgetary funds for hiring additional personnel who could assist with 
classroom coverage, the process of teacher collaboration by means of classroom observations may not be feasible. Teacher collaboration was one way that participants were able to gain more knowledge about their focal students, but there were many other ways they were able to do so on their own.

\section{Home Visits}

Participants recognized that home visits have the potential to provide them with a lot of information; yet, they shared trepidations about the process. Of the five participants, four had some type of experience with visiting a student's home; however, the extent of experience ranged from one participant's experience dropping off a backpack from the food program and leaving without interacting with the parents to another participant's extensive experience with visiting families at their homes for a variety of purposes and attending students' activities outside of school. Although experience with home visits was not uniform, all participants, aside from the teacher with extensive home visit experience, expressed interest in obtaining more information about conducting home visits as well as interest in opportunities for receiving training or coaching in order to have their questions about home visits answered. Participants expressed interest in having "parameters," "expectations," "guidelines," or "steps and strategies kind of like a blueprint." Teacher participants wanted to learn from individuals who have conducted home visits who might be able to offer advice or suggestions for effective practices. Participants also indicated that parameters and expectations set forth by the district would make the practice of home visits more "comfortable." Examples of desired parameters included how many students to visit, how to select which students to 
visit, how long visits should last, an established purpose or goal for the visits, how to initiate or set up a home visit, and how to maintain teacher safety during a home visit.

The interest in receiving training corresponds with participants' reservations about conducting home visits. Although each participant indicated that she either already had or would be willing to do a home visit, many factors were identified as barriers or concerns about the process. Participants identified discomfort, safety, parent reactions, and language barriers as concerns in addition to questioning if home visits would be conducted inside or outside of work hours and whether or not they would be compensated for their time spent conducting home visits. The most pervasive concerns about home visits shared among the majority of participants were personal discomfort and worrying about doing something that would be offensive to parents. Even Monica, who had extensive experience with home visits and who was comfortable with the process spoke about an occasion where she experienced discomfort visiting a student's home.

Participants were willing to overcome their discomfort based on their ideas about the benefits of home visits; yet, they shared the feeling of hesitance due to concern about offending parents or not being well received by parents when they try to initiate a home visit. Linda expressed interest in learning more about the cultural backgrounds of her students in order to have an understanding of what having "manners" would look like in a traditional Hispanic home or traditional Marshallese home so that she, too, might exhibit appropriate manners. The consensus among participants was that they would not feel comfortable initiating or conducting home visits until they were prepared with a better understanding of how to do them effectively. 
Despite their concerns about home visits, participants had a shared vision of what their goals would be in conducting home visits and, in most cases, identified the same intentions as their top two goals. Participants indicated that if they were to conduct home visits, they would hope to gather information about students and their families, which was described as "spying," "observing," "collecting below the line information," "getting to know the student and their family a little better and learning more about them," and "seeing what the inside of their home looks like on a normal day." They also saw home visits as a way to build relationships with students and their families, which one participant described as "making the parent feel comfortable with me and willing to approach me." They also agreed that the information gathered from home visits would be very valuable in terms of better understanding their students. Monica described a home visit as "a million points of input" and said, "The home is the photograph." Brenda indicated that "seeing the way a child is comfortable in their own home," and knowing "what conditions they're living in" could give her valuable information about that child. Mary added that a home visit allows a teacher to notice things about a student's environment that a parent may not have shared due to not thinking it was relevant or due to feeling embarrassed about it. She gave the examples of not having his or her own room or sleeping on a couch as something that wasn't likely to come up during a conference at school, but as something that could give her "information about what's going on for that child." In the case of a parent feeling embarrassed about some aspect of their home life, a teacher visiting the home could obtain first hand observational knowledge, yet would not 
have to specifically question a parent or draw attention to something the parent did not wish to talk about.

Even though participants shared goals for home visits that affirm the importance of learning from and about students and their families, most participants shared that they would feel more comfortable approaching a home visit if they had a particular task to accomplish, such as getting a form signed or dropping off the backpack full of food that a child didn't receive at school due to being absent. One participant said that she would feel more comfortable having a questionnaire or something "to do" in order to have a purpose that was more "tangible" for both herself and the parents.

Discussion. Participants' discussions about home visits were marked by a discrepancy between what they stated as their intended objectives for home visits and their comfort level with going into homes with the open-ended purpose of gathering information and building relationships. Based on the current comfort level of most participants, training sessions or guidelines for conducting home visits would be beneficial. However, because most participants felt that teachers would not be receptive to home visits being made mandatory, I think it would be very important for any training or professional development opportunities about home visits to be optional for teachers who were interested. I am confident that through learning about home visits and receiving training on how to effectively initiate and conduct home visits, teachers could increase their comfort level with the practice. That being said, I think that a certain level of discomfort is to be expected regardless of the extent of prior training; yet, the overall benefit of the visit outweighs the discomfort that may be experienced. Although there 
cannot be a precise set of directions for how to conduct a home visit due to the variations in family dynamics, family to school dynamics, and teacher to student dynamics, preparing for home visits by means of hearing advice from teachers who have conducted them or learning about traditional cultural customs in order to avoid being offensive would certainly benefit teachers interested in decreasing their worry or misgivings.

\section{Summary}

Participants experienced the CARE process in unique ways, but they also shared common perspectives about culturally responsive scripted curricula, relationship building as a key aspect to better understanding culturally and linguistically diverse students, teacher collaboration, and home visits. The subsequent chapter connects the findings from the study to assertions about the interplay between culturally responsive instruction and curricula that contribute to answering the research questions. 


\section{CHAPTER V}

\section{DISCUSSION AND CONCLUSIONS}

This chapter begins with a summary of the findings and themes that emerged from the cross-case analysis of the study. Further reflection and assertions as well as discussion of the findings within the greater context of education for culturally and linguistically diverse students are then followed by recommendations for both future research and future practice.

\section{Summary of the Study}

The goals of this study were to identify and understand the behaviors and ideas teachers have developed as ways to connect with their culturally and linguistically diverse students and their families. Additionally, the study investigated how elementary teachers who implement scripted curricula describe the experience of creating culturally responsive lessons intended to specifically connect with their culturally and linguistically diverse students and how teachers incorporate students' funds of knowledge into their instruction. By sharing about their focal students, and talking through their CARE lessons, participants were able to give voice to the process of merging culturally responsive instruction with scripted instruction.

A multiple case study approach was employed to collect data from five teachers who participated in CARE training during the 2011-2012 school year. Data was organized for analysis using HyperRESEARCH software and was presented by focusing specifically on each case to showcase each participant's unique experience as well as by 
examining themes that emerged across cases. The cross-case analysis brought forth the following themes:

- Feasibility of culturally responsive instruction while maintaining the integrity of scripted curricula, or "culturally responsive scripted instruction."

- Relationship building as key for better understanding culturally and linguistically diverse students.

- Teacher collaboration as a beneficial practice for improving culturally responsive instruction.

- Appreciation for the value of home visits paired with apprehension about the process and the desire for training.

The preceding themes, informed by data in this study, led to the following assertions:

1. The use of scripted curricula may inhibit teachers' abilities to incorporate ethnic and culturally diverse curricular content as an aspect of culturally responsive teaching.

2. Attending to the specific communication styles and learning styles of culturally and linguistically diverse students does not impede teachers' ability to maintain the integrity of the scripted-curricula.

3. Relationships between culturally and linguistically diverse students and their teachers play a key role in teachers being able to determine which instructional strategies or curricular modifications will be appropriate for the students. 
4. Teacher collaboration, in the form of peer observations, helps facilitate better understanding of culturally and linguistically diverse students because an observing teacher can attend to nuances that an instructing teachers may not notice.

5. Home visits have potential for helping teachers better understand their culturally and linguistically diverse students.

6. Lack of preparation and concerns about the feasibility of conducting home visits invites the need for explicit training in the area of home visits.

Table 10 illustrates how the themes from the cross-case analysis connect to the six assertions. 
Table 10

Summary of the Relationship Between Cross-Case Themes and Assertions Cross-Case Themes Assertions

Culturally Responsive Implementation of Scripted Curricula

Relationships as a Key to

Better Understanding

Culturally and Linguistically

Diverse Student

Teacher Collaboration

Home Visits
1. The use of scripted curricula may impede teachers' abilities to incorporate ethnic and culturally diverse curricular content as an aspect of culturally responsive teaching

2. Attending to the specific needs and learning styles of culturally and linguistically diverse students does not impede teachers' ability to maintain the integrity of the scripted-curricula

3. Attending to the specific needs and learning styles of culturally and linguistically diverse students does not impede teachers' ability to maintain the integrity of scripted curricula

4. Teacher collaboration, in the form of peer observations, helps facilitate better understanding of culturally and linguistically diverse students because an observing teacher can attend to nuances that an instructing teachers may not notice.

5. Home visits have potential for helping teachers better understand their culturally and linguistically diverse students.

6. Lack of preparation and concerns about the feasibility of conducting home visits invites the need for explicit training in the area of home visits.

Each portion of data analysis contributed uniquely to explaining and addressing the study's research questions. The individual case narratives and inclusive themes 
provide a detailed account of each participant's perspective through a comprehensive story. The cross-case themes highlight participants' common experiences and represent a broader look at consistencies across the collective group of participants. Finally, the assertions bring forth more generalized statements, or conclusions, from the study that specifically inform the answers to the research questions. Table 11 includes a brief, summative answer to each of the five research questions.

Table 11

Summary of Answers to the Study's Research Questions

\section{Key Words from Research Question}

R.Q.1.

R.Q.1a.

R.Q.1b.

R.Q.2.

R.Q.2b.

\section{Brief Answer}

Participants were able to utilize culturally responsive instructional strategies in conjunction with the implementation of scripted curricula.

Participants provided examples of how they utilized below the line information about students to inform their instruction; yet, they did not provide evidence of incorporating students' funds of knowledge.

Participants perceived that their use of culturally responsive instructional strategies resulted in increased engagement from their focal students.

Participants emphasized that they built relationships with the focal students in order to learn more about them.

Participants expressed appreciation for the value of home visits; yet, they shared many concerns about the process and desired training and/or parameters to gain a better understanding of how to conduct them 


\section{Discussion}

Based on the shared perspectives held by participants regarding the overarching research questions and some of the topics that were studied, it follows that merging the distinct theoretical ideologies that support the implementation of research-based scripted curricula and culturally responsive instruction is very feasible. Participants provided context for how they negotiated using district-adopted scripted curricula with integrity while also attending specifically to their focal students. They also emphasized the significance of building relationships, which is an appropriate action for any teacher in any situation. Due to the subjective nature of culturally responsive instruction, participants' ideas and examples from their practice clearly cannot simply be replicated. However, their examples do provide a basic blueprint for how teachers can begin to approach their desire to be culturally responsive regardless of what type of curriculum is already in place. The investigation of the potential interplay between scripted curricula and culturally responsive instruction also raises some topics that invite additional discussion and clarification.

\section{Fidelity vs. Integrity}

The term fidelity, just like many other words used in academic arenas, is interpreted and used differently by professionals. In my teaching experience, the term fidelity, especially when used by superiors in my district, implies a strict adherence to a scripted program. Dewitz et al. (2010) reference Linda Diamond of the Consortium on Reading Excellence to provide a detailed description of what is meant by fidelity. Diamond said: 
High fidelity implementation means that you get a program with an internal design and follow that design. That would include using the materials in a particular sequence, adhering to the amount of time and practice called for by the program and following the recommendations for grouping and re-teaching students. It would mean using all of the essential components as they are designed (p.311).

Although participants perceived that they were still utilizing scripted curricula with fidelity, according to Diamond's description their modifications to activities, pacing, and lesson delivery violated the criteria for maintaining fidelity, which carries a negative undertone. Yet, Dewitz et al. point out that:

The teacher's edition suggests many options for how to use the core program without strict fidelity, and these options are available because a core reading program must appeal to many different instructional audiences and meet the needs of many different types of readers. Teachers must rely on their knowledge of curriculum and instruction, their understanding of children, and the goals of their school or district to select and design instruction that helps students meet these goals (p.313).

I appreciate the aforementioned point because it recognizes the importance of professional knowledge, the teacher's role as expert about or her particular students, and the need for teachers to reflect on the content of the scripted program rather than simply delivering it exactly as written, all of which coincides with the essential focus of culturally responsive instruction and CARE work. Therefore, instead of critiquing whether or not teachers truly were implementing their scripted curricula with fidelity, my preference is to refer to participants' ideas and intentions regarding keeping the objectives, vocabulary, and skills identified for instruction intact as teaching the scripted curricula with integrity. 
Based on their efforts to not make changes to what Linda referred to as the "heart" of the lessons or the main structure of the programs they used, I see these teachers as maintaining the integrity of the research base and, therefore, the integrity of the program design. Yet, participants also attended to their specific audiences, utilized their professional knowledge, and took the below the line information they learned about their students into consideration when planning their lessons. I do not equate integrity with fidelity; however, I would venture to say that using a scripted program with integrity, although not as close to the exact intended use, is superior to using it only as written or not using it at all when attending to cultural responsiveness. Therefore, I believe that participants enhanced the curriculum by making the necessary adjustments to engage their focal students without detracting from the main content, which I think is more effective for culturally and linguistically diverse students than teaching the program strictly according to the aforementioned definition of fidelity. Participants demonstrated thoughtful and professional decision-making with the best interests of their focal students in mind. Additionally, they relied on their colleagues to provide them feedback, providing more opportunities for reflection on their practice.

\section{Potential Limitations to Culturally Responsive Scripted Instruction}

Because the majority of literature on multicultural education and culturally responsive/relevant instruction is not contextualized within the use of scripted curricula, it is important to synthesize how the findings from this study fit within the overall context of curriculum and instruction for culturally and linguistically diverse students. Gay (2010), Banks (2001), and Ladson-Billings (1995) each offer a unique summation of the 
critical features of education for culturally and linguistically diverse students. Table 12 revisits the comprehensive look at the components of culturally responsive instruction according to Gay and the features of culturally responsive instruction were evident in participants' discussions of their CARE lessons. Although the discussion and analysis from this study forefront Gay's work, I have also included the work of Banks to provide additional contextualization of the possible limitations associated with the interplay between scripted curricula and culturally responsive instruction. Because this study primarily focuses on teachers' use of culturally responsive teaching practices, I did not include the components as described by Ladson-Billings because her tenets focus on the student experience compared to Gay and Banks who emphasize the teacher's role in educating culturally and linguistically diverse students. 
Table 12

How This Study's Findings Represent the Components of Culturally Responsive Instruction and Multicultural Education as Described By Gay and Banks

Culturally Responsive Instruction

Gay

(2010)
Multicultural Education

Banks

(2001)

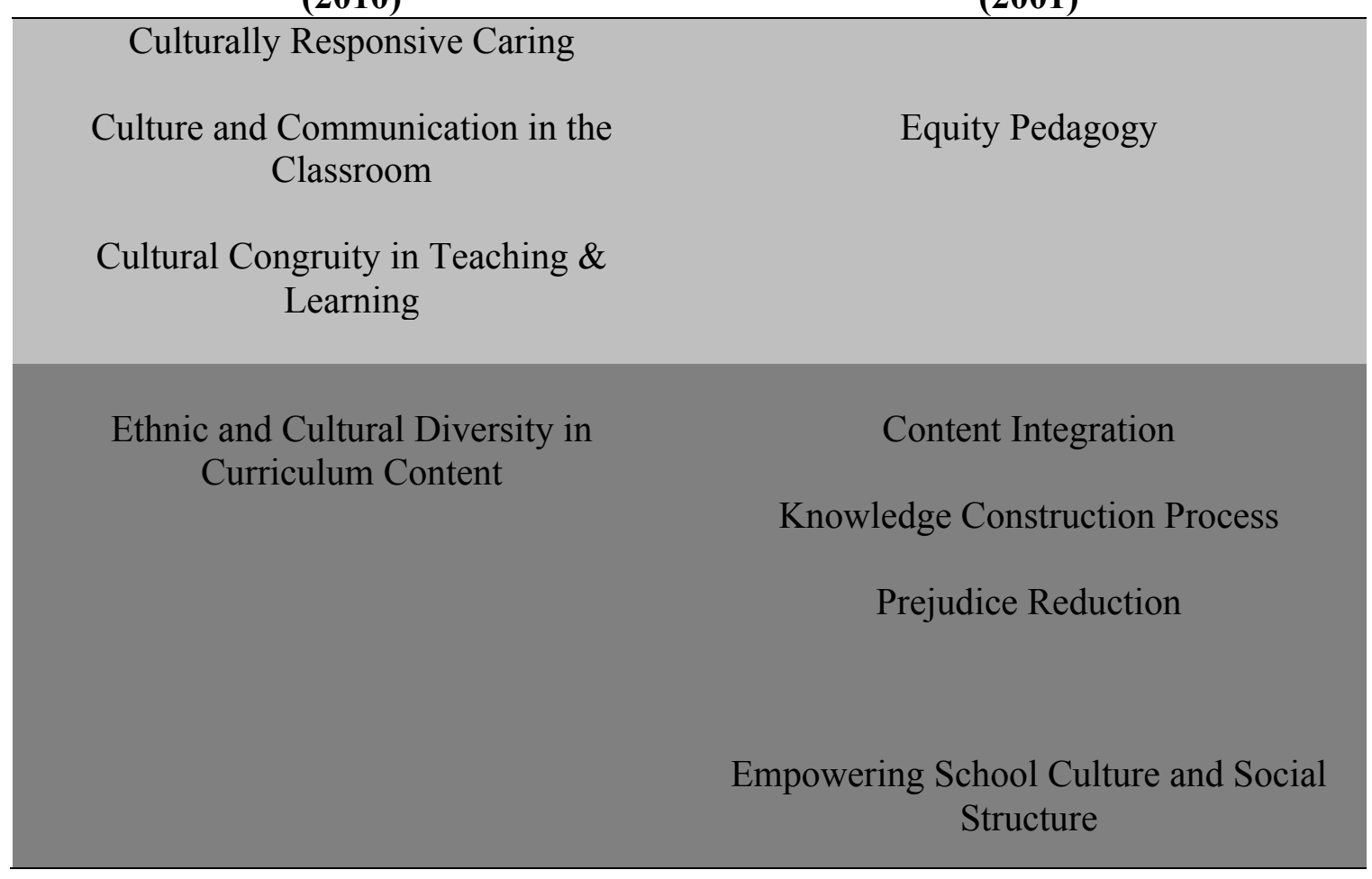

KEY

Represented in this study's findings

Not represented in this study's findings

Table 12 is color-coded to illustrate that based on participants' reports they employed culturally responsive caring, culturally responsive communication in the classroom, and cultural congruity in teaching and learning, which collectively correspond with Banks' equity pedagogy. However, given their inclination to teach district-adopted curricula with 
integrity, they did not significantly alter the curricular content to reflect students' funds of knowledge, which both Gay (ethnic and cultural diversity in curriculum content) and Banks (content integration) address as a significant component of instruction for culturally and linguistically diverse students.

Ethnic and cultural diversity in curriculum content is likely the most challenging of Gay's (2010) components to accomplish, particularly when implementing a scripted curriculum that already includes the curricular content to be taught. Therefore, it should be noted that culturally responsive instructional strategies used in conjunction with the implementation of scripted curricula might interfere with teachers' abilities to incorporate each of the components of culturally responsive instruction equally. It follows that the extent to which teachers incorporate ethnically and culturally diverse curriculum content may affect the extent to which they can implement the scripted curricula with integrity. Or, when approached from another side, if teachers continue to implement scripted curricula with integrity, they are limited in the extent to which they can employ culturally responsive instruction.

Based on my own experience trying to incorporate students' funds of knowledge into scripted curriculum content, I do believe that teachers who use scripted programs can incorporate ethnic and culturally diverse content to a greater extent than was represented in this study's findings. I tried several different ideas and activities with my Kindergarten students in order to make my instruction more culturally responsive while maintaining the integrity of the district adopted scripted curricula. One example is that I used personal alphabets with my scripted phonics program to help students relate letter sounds to 
important people, places, things, and experiences in their own lives (Toppel, 2012) and found that this very simple addition helped me to learn more about my students and helped them feel connected to my instruction when I read their words.

The difficulty of incorporating ethnic and cultural diversity into curriculum content will likely differ based on the grade level of the students, the subject being taught, and the particular content within the subject area. Yet, I believe the biggest challenge associated in achieving ethnic and cultural diversity in curriculum content, regardless of the type of curriculum used (i.e. scripted versus non-scripted), is how teachers go about becoming familiar with students' funds of knowledge and how well teachers understand the concept of funds of knowledge.

\section{Below the Line Information vs. Funds of Knowledge}

Several teachers expressed interest in knowing more about their focal students than they were able to discover through their interactions with those students at school, which led me to question if my initial decision to equate funds of knowledge with below the line information was misguided. I still think there is a significant overlap between the two terms; yet, after analyzing the data and reflecting further, I believe there is a fundamental difference between the two, which I would ascribe to the difference between the words "information" and "knowledge." Below the line information could be any information about a student or their family, ranging from the fact that the student likes soccer to the fact that the student is tired in class because he stays up late attending his dad's soccer games. Yet, the term funds of knowledge has more to do with how the child understands the world based on his or her daily living experiences. The concept of funds 
of knowledge is grounded in what they know as a result of being in their particular family and involves a sense of longevity in terms of the student's family history as opposed to something situational about the child that may change from month to month or from year to year. I would consider funds of knowledge to be a specific subset of the broader category of below the line information, which is depicted in Figure 4.

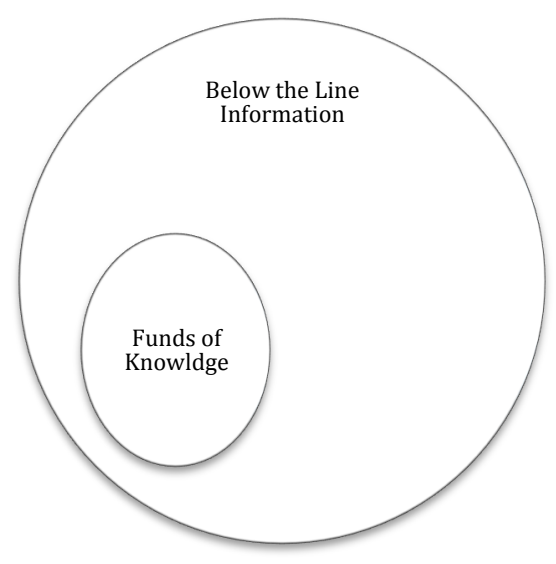

Figure 4. Relationship Between Funds of Knowledge and Below the Line Information Discovering that a student is crazed about Star Wars and will complete extra math work in exchange for a Darth Vader coloring page does not align with knowing that a student's mother, aunts, and grandmothers all practice midwifery and that the student has witnessed births and helped care for newborn babies. Knowing that a child is very shy and prefers to collaborate with students that speak her native language differs from knowing that a child belongs to a family of farmers and has extensive knowledge about planting, harvesting, and selling produce. Both kinds of information are valid; yet, I believe the type of information about students and their families that is associated with funds of knowledge is the type of information that teachers need in order to create 
ethnically and culturally diverse curriculum content as opposed to modifying instruction to make it more engaging. It is possible that the lack of evidence relating to ethnic and cultural diversity in curriculum content in this study may have been a result of my equating below the line information with funds of knowledge. I will further discuss how the synonymous use of those terms may have influenced the absence of ethnic and cultural diversity in curriculum content in the section on limitations.

This distinction between below the line information and funds of knowledge causes me to resituate funds of knowledge as information likely contained within the contexts of students' homes and circles back to home visits as a viable way to provide teachers with opportunities to connect with students' funds of knowledge. Participants recognized that home visits have the potential to provide them with a unique type of information; yet, they shared trepidations about the process. If teachers are not conducting home visits, or conducting them on a limited basis, it follows that their access to students' funds of knowledge may be limited unless they can find other ways to connect with students and their families to bring forth that information.

\section{Limitations}

This study primarily addressed teachers' perceptions about the interplay between scripted curricula and culturally responsive instruction in addition to the ideas and behaviors teachers have for better understanding culturally and linguistically diverse students. The emphasis was on CARE teachers' thoughts, reflections, and descriptions of their actions pertaining to how they crafted culturally responsive instruction in conjunction with the use of district adopted scripted curricula. Results indicated teachers 
perceived that their culturally responsive pedagogy positively influenced their focal students' engagement and the nature of their relationships with their focal students. However, the study did not operationalize and measure engagement nor did it address students' perspectives, both of which are very pertinent for the field of multicultural education. Participants mentioned that their focal students became more engaged as a result of the CARE lessons that utilized culturally responsive instructional strategies; yet participants did not share enough details about students' engagement to understand specifically how it changed. Therefore, the claim that the CARE lessons positively influenced students' engagement is lacking evidence to support teachers' perceptions that improvement in engagement actually occurred.

District CC has done extensive work with various trainings spotlighting race in education; therefore, the results may not apply to other districts where teachers are not as advanced in processing how race impacts students and learning in the classroom or who are not as informed about or focused on culturally responsive instruction. It is always beneficial to draw conclusions that are applicable across multiple settings and circumstances; yet, that was not the intention of this study. The data collected will provide valuable information to District CC as well as to the existing body of knowledge about teachers' experiences in creating culturally responsive lessons. A case study design is not used with the intention of uncovering broadly generalizable data; rather the case study design is selected to elicit a very detailed account from a small number of cases. 
Finally, the choice to equate the concepts of below the line information and funds of knowledge at the outset of the study may have contributed to the underrepresentation of funds of knowledge in participants' CARE lessons, and therefore, the underrepresentation of ethnic and culturally diverse curriculum content. I used the term funds of knowledge in my research questions because that specific term was used in the literature that I read. Yet, when I wrote my interview questions, I used the term below the line information because that was the term used throughout CARE training that participants would be more familiar with. At the outset of the study, I did not reflect specifically on the ways in which those terms may be different and in retrospect, I think it would have benefitted the work to do so.

Because the two terms have very similar meanings, I did not take the time to explain the term funds of knowledge or to unpack with participants what about that term funds of knowledge is distinct from below the line information. Consequently, an opportunity to speak with them and question them about funds of knowledge was lost. The type of the information participants collected about their focal students allowed them to make adjustments to their instruction in efforts to better engage those students. Because teachers were focusing on how to better engage their focal students, it is likely that they sought out information that would help them to do so (i.e. Monica listening to her students' favorite radio stations to find songs for math raps). Had participants been focused on collecting examples of students' funds of knowledge, it is likely that those examples may have been utilized in CARE lessons to represent ethnically and culturally diverse curriculum content. 


\section{Recommendations}

Based on the findings and conclusions of this study, I have recommendations in the areas of future research, in-service professional development for teachers, the use of culturally responsive instructional strategies combined with the implementation of scripted curricula, and home visits.

\section{Future Research}

Because this study was conducted within one moderately sized school district and data was collected from five teachers, replication of the study in other districts, with different teachers, might provide additional insight into how teachers who use scripted curricula can better meet the needs of their culturally and linguistically diverse students. I have a few recommendations for replication that might improve the richness and depth of data.

Rather than collecting data after teachers have completed CARE training, I think it would be more beneficial for the series of interviews to be completed concurrently with the training because the content of the trainings and the CARE lessons would be more recent. The timing of participant recruitment in this study and interviewing teachers over summer break was not ideal because not all participants provided CARE lesson plans and they also struggled to remember particular details about their lessons and their focal students. Additionally, recruiting participants much earlier in the school year might result in the recruitment of a larger number of interested participants, which would then enable the researcher to utilize selection criteria to ensure a greater variety among participants in terms of grade level taught, years of experience, and gender. Finally, because not all 
participants provided written documentation of their CARE lessons and they experienced difficulty recalling the exact details of how they planned and implemented the lessons, I would recommend including lesson observations in any subsequent studies investigating the research questions that were addressed in this study. By observing CARE lessons, the researcher could have an opportunity to gain firsthand knowledge of the lesson content, which might help elicit more thoughtful and detailed descriptions from participants.

The overarching ambitions behind the study were a) to fill a gap in the current literature pertaining to how culturally responsive instruction and scripted curriculum might fit together and b) to explore if accessing students' funds of knowledge can assist teachers to improve educational outcomes for culturally and linguistically diverse students. Yet, a study focusing on how teachers get to know culturally and linguistically diverse students and consequently alter their instruction to meet the needs of those students leaves space for additional research that focuses more explicitly on how culturally responsive instruction affects students for whom it is meant to benefit.

Literature does address the impact culturally responsive instruction has on culturally and linguistically diverse students (Bell \& Clark, 1998; Hefflin, 2002; Howard, 2001; Ladson-Billings, 1992b; Powell, 1997), but studies that contextualize culturally responsive instruction within the use of scripted curricula are lacking. Research in the area of multicultural education has more recently expanded to address cultural responsiveness as it relates to explicit instruction (Pace, 2011) as well as research-based instruction (McIntyre, Hulan, \& Layne, 2011; McIntyre \& Hulan, 2013). Yet, research specifically addressing culturally responsive instruction as it merges with scripted 
curricula is important for understanding the potential this combination has for culturally and linguistically diverse students. Studies that investigate how culturally instructional practices influence student achievement, student motivation, student engagement, and students' sense of belonging in schools will add depth to the current literature in the field. Test scores and numerical data should not be the only criteria used to determine student achievement because teachers have detailed knowledge about their students' progress that may not be reflected in such data; yet similarly, the field cannot rely entirely on teachers' perspectives about how culturally and linguistically diverse students are impacted without additional data to support their claims.

This research suggests that culturally responsive practices can be used in conjunction with scripted curricula while maintaining the integrity of the prescribed program. Additional research that explicitly addresses how teachers go about this process and that incorporates a direct focus on outcomes relating to student engagement, student motivation, and student achievement will contribute to further contextualizing the significance and feasibility of culturally responsive instruction

\section{In-Service Teacher Professional Development}

The Courageous Conversations (Singleton \& Linton, 2006) trainings that District $\mathrm{CC}$ has participated in for the past several years are an excellent platform for professional development in the area of diversity as well as cultural and racial awareness. The Beyond Diversity workshop in addition to the four agreements and six conditions are first-rate groundwork for helping teachers understand the role race plays in schools as institutions. The work engaged in throughout the CARE process guides teachers to learn more about 
their culturally linguistically diverse students in order to employ culturally responsive instructional strategies that emphasize rigor, relevance, relationships, and realness in curriculum and instruction.

I attended a teacher education program that did not emphasize diversity or how race and culture influence both teaching and learning. Consequently, participating in the various aspects of Courageous Conversations, particularly the CARE process, has fundamentally impacted the way I know and understand my students and the way that I teach. The professional development opportunities put forth by the Pacific Educational Group (PEG) have the potential to greatly influence how teachers understand and process issues about race, culture, equity, and diversity as they relate to teaching, learning, and curriculum.

Despite my positive experience with CARE and other PEG trainings, much of my learning stemmed from applying the doctoral work that I was also doing to the content that PEG facilitators were presenting. The idea for this study emerged from my own authentic questions relating to how I could utilize what I learned in CARE trainings while at the same time trying to implement the district-adopted scripted curricula with fidelity. Consistently throughout the year-long CARE experience, I often felt a tension between the mandate to teach the adopted curricula with fidelity, which meant not altering it, and the CARE work requiring me to examine my practice to ensure that it was appropriate for my focal students, which required making changes to prescribed lessons. Not only did I sense a disconnect between the literature on culturally responsive instruction and implementing scripted curricula, but I also felt a disconnect between what I was learning 
about in the professional development series and what I was supposed to do in the classroom. I felt the need for explicit attention to the intersection between culturally responsive instruction and the use of scripted curricula, which was not addressed in the training. I did not feel as though I could fully understand and begin to implement culturally responsive instructional strategies until I could understand how to incorporate them into the use of prescribed lessons.

Consequently, I believe professional development training will not contribute to changing and improving teacher practice if the new content is not clearly connected to and/or combined with the teaching reality that teachers face when they are teaching students, which includes, but is not limited to, the type of curricula they use. Enhancement and modification of scripted curricula will not follow a particular formula because each teacher will modify the programs to better engage his or her particular students, but professional development opportunities need to address how to create an interplay between utilizing the scripted programs with integrity while concurrently honoring the needs of students. As a "rule-following" teacher, I want to know that I have support from administration to make program adjustments and hope to have guidance in moving away from the "one size fits all" mentality.

\section{Culturally Responsive Teaching Combined with Scripted Curricula}

Not all districts and schools use scripted curricula; however, the use of such programs is widespread (Dewitz et al., 2010), meaning that many teachers will encounter the challenge of teaching in culturally responsive ways while implementing prescribed programs. Teachers need to have the understanding that despite the call for 
standardization, instruction will change from year to year because it must be tweaked to fit each unique group of students. Therefore, even when teachers are implementing scripted programs, they should have knowledge of how to utilize the research-based best practices contained in the programs while also attending to the needs of their students and tweaking/enhancing the curricula as they see fit.

As a teacher who entered the profession right when NCLB was enacted, I am part of a generation of teachers who have experienced the directive to implement scripted curricula with fidelity. Compared to my colleagues who have been in the field for more time, I do not have as much experience with curriculum planning because I have always worked in educational settings where I was responsible for implementing a curriculum that was already planned, sequenced, and packaged. I have not been afforded opportunities to create my own curriculum, and consequently, I have held tight to the mandate that I should teach the district-adopted curricula with fidelity without creating my own lessons or straying from the scope and sequence.

However, perhaps as a result of the extensive work with PEG and CARE training, the educational climate in my district now seems to be shifting to reflect a push for reflective practice and instruction based more on the needs of particular students as opposed to instruction based solely on implementing the lessons in scripted programs exactly as they are written. This shift creates a need for teachers to have the time and space to work with colleagues on instructional planning and for teachers to be capable of knowing how to work with a scripted curriculum in ways that honor what the curriculum 
offers while at the same time acting as an expert on the particular students the curriculum is being used for.

\section{Home Visits}

Regarding the prospect of home visits to inform culturally responsive instruction, I hesitate to say that districts should add mandatory home visits to teachers' already filled agendas. Rather, I think that school districts could capitalize on the knowledge of teachers who have benefited from home visits and offer trainings for teachers who are interested. I believe interest and investment on the part of teachers is essential in order for home visits to have positive outcomes, therefore doing them should not be forced upon them. Yet even interested teachers who are motivated to begin conducting home visits face barriers that were addressed by participants in this study such as safety, time, and concerns about parents' reactions to home visits.

I don't foresee home visitation becoming a mandated or widespread practice among elementary teachers; yet, I do believe that even a small number of teachers engaging in the practice can make a different for some students and their families. The teachers who continue or begin to visit students' homes because they believe in the purpose and the value in them will most likely be the teachers that those students remember and feel most connected with. If a single teacher can create a relationship with a single student that motivates that student to stay in school or makes that student excited about learning, then the investment was worthwhile. 


\section{Conclusions}

In an era where the number of culturally and linguistically diverse students is increasing rapidly (Ginsberg, 2007; Schmidt \& Lazar, 2011), culturally responsive instruction is imperative. Teachers need to be capable of enhancing curricular content and instructional practices to reflect and engage the diverse populations of students that inhabit their classrooms. Whether teachers are free to plan and create their own curriculum or are mandated to implement a district adopted scripted curriculum, it is essential they do so with regard for their students' various ethnic and cultural affiliations in order to prevent outcomes for their students that are stratified based on those very characteristics. Particularly when teachers' backgrounds differ from those of their students, they must work hard to get to know their students and to build relationships with their students so they are capable of making informed decisions regarding the best way to facilitate school success. 


\section{REFERENCES}

Baeder, A. (2010). Stepping into students' worlds. Educational Leadership, 67(5), 56-60.

Banks, J.A. (1999). An Introduction to Multicultural Education. Boston, MA: Allyn and Bacon.

Banks, J.A. (2001). Cultural Diversity and Education: Foundations, Curriculum, and Teaching $\left(4^{\text {th }} \mathrm{ed}\right)$. Boston: Allyn and Bacon.

Bell, Y.R., \& Clark, T.R. (1998). Culturally relevant reading material as related to comprehension and recall in African American children. Journal of Black Psychology, 24(4), 455-475.

Benson, B.E. (2003). Framing culture within classroom practice: Culturally relevant teaching. Action in Teacher Education, 25(2) 16-21.

Boske, C.A., \& Benavente-McEnery, L. (2010). Taking it to the streets: A new line of inquiry for school communities. Journal of School Leadership, 20(3), 369-398.

Chall, J.S. (1996). Learning to Read: The Great Debate. $\left(3^{\text {rd }}\right.$ ed.). New York: Harcourt Brace College Publishers.

Creswell, J.W. (2007). Qualitative Inquiry and Research Design: Choosing Among Five Approaches $\left(2^{\text {nd }}\right.$ ed.). Thousand Oaks: Sage Publications.

Delpit, L. (2012). Multiplication is for White People: Raising Expectations for Other People's Children. [Kindle]. Retrieved from www.amazon.com

Dewey, J. (1902). The Child and the Curriculum. Chicago: The University of Chicago Press.

Dewitz, P., Leahy, S.B., Jones, J., and Sullivan, P.M. (2010). The essential guide to selecting and using core reading programs. Newark, DE: International Reading Association, Inc.

Dey, I. (1993). Qualitative Data Analysis: A User Friendly Guide for Social Scientists. New York: Routledge Taylor \& Francis Group.

Duncan-Owens, D. (2009). Scripted reading programs: Fishing for success. Principal, 26-29. 
Eisenbach, B.B. (2012). Teacher belief and practice in a scripted curriculum. The Clearing House: A Journal of Educational Strategies, Issues and Ideas, 85(4), 153-156.

Eliot \& Associates. (2005). Guidelines for Conducting a Focus Group.

Fang, Z., Fu, D., \& Lamme, L.L. (2004). From scripted instruction to teacher empowerment: Supporting literacy teacher to make pedagogical transitions. Literacy, 38(1), 58-64.

Freire, P. (2007). Pedagogy of the Oppressed. New York: Continuum International Publishing Group, Inc.

Gay, G. (2002). Preparing for culturally responsive teaching. Journal of Teacher Education, 53(2), 106-116.

Gay, G. (2010). Culturally Responsive Teaching: Theory, Research, and Practice $\left(2^{\text {nd }}\right.$ ed. $)$. New York: Teachers College Press.

Ginsberg, M.B. (2007). Lessons at the kitchen table. Educational Leadership, 64(6), 56-61.

González, N., Moll, L.C., \& Amanti, C. (Eds.). (2005). Funds of Knowledge: Theorizing Practices in Households, Communities, and Classrooms. Mahwah, NJ: Lawrence Erlbaum Associates, Publishers.

Goldenberg, C. (2008). Teaching English language learners: What the research does-and does not-say. American Educator, 8-44.

Grant, C.A., \& Sleeter, C.E. (2003). Turning on Learning: Five Approaches for Multicultural Teaching Plans for Race, Class, Gender, and Disability ( $3^{\text {rd }}$ ed.). Danvers, MA: John Wiley \& Sons, Inc.

Guba, E.G. (1990). The Paradigm Dialog. Newbury Park, CA: Sage Publications, Inc.

Hefflin, B.R. (2002). Learning to develop culturally relevant pedagogy: A lesson about cornrowed lives. The Urban Review, 34(3), 231-250.

Hirsch, E.D. (2007). Teaching content is teaching reading. Principal, 90(20), 10-14.

Howard, T.C. (2001). Powerful pedagogy for African American students: A case of four teachers. Urban Education, 36(2), 179-202. 
Howard, T.C. (2010). Why Race and Culture Matter in Schools: Closing the Achievement Gap in America's Classrooms. New York: Teachers College Press.

Johnson, B., \& Christensen, L. (2008). Educational Research: Quantitative, Qualitative, and Mixed Approaches. $\left(3^{\text {rd }}\right.$ ed.). Los Angeles: Sage Publications.

Kitzinger, J. (1994). The methodology of focus groups: The importance of interaction between research participants. Sociology of Health and Illness, 16(1), 103-121.

Kliebard, H.M. (2004). The Struggle for the American Curriculum. ( $3^{\text {rd }}$ ed.). New York: RoutledgeFalmer.

Ladson-Billings, G. (1992a). But that's just good teaching. Theory into Practice, 34(3), 159-165.

Ladson-Billings, G. (1992b). Liberatory consequences of literacy: A case of culturally relevant instruction for African American students. The Journal of Negro Education, 61(3), 378-391.

Ladson-Billings, G. (1995). Toward a theory of culturally relevant pedagogy. American Educational Research Journal, 32(3), 465-491.

Ladson-Billings, G. (1999). Dreamkeepers. San Francisco: Wiley \& Sons, Inc.

Lazar, A.M. (2011). Access to excellence: Serving today's students through culturally responsive literacy teaching. P.R. Schmit \& A.M. Lazar, (Eds). New York: Teachers College Press.

Learning Points Associates. (2007). Understanding no child left behind. Naperville, IL.

Lin, M., \& Bates, A.B. (2010). Home visits: How do they affect teachers' beliefs about teaching and diversity. Early Childhood Education Journal, 38, 179-185.

Lincoln, Y.S., \& Guba, E.G. (1985). Naturalistic Inquiry. Newbury Park, CA: Sage.

Maxwell, J.A. (2005). Qualitative Research Design: An Interactive Approach (2 ${ }^{\text {nd }}$ ed.). Thousand Oaks: Sage Publications.

McIntyre, E., \& Hulan, N. (2013). Research-based, culturally responsive reading practice in elementary classrooms: A yearlong study. Literacy Research and Instruction, 52(1), 28-51. 
McIntyre, E, Hulan, N, \& Layne V. (2011). Reading Instruction for Diverse Classrooms. New York: The Guilford Press.

Meyer, J.A., \& Mann, M.B. (2006). Teachers' perceptions of the benefits of home visits for early elementary children. Early Childhood Education Journal, 34(1), 93-97.

Moll, L.C., Amanti, C., Neff, D., \& Gonzalez, N. (1992). Funds of knowledge for teaching: Using a qualitative approach to connect homes and classrooms. Theory into Practice, 31(2), 132-141.

Morgan, D.L. (1996). Focus groups. IN J. Hagan \& K.S. Cook (Eds.), Annual review of sociology (Vol.22, pp.129-152). Palo Alto, CA: Annual Reviews.

Morgan, D.L. (1997). Focus Groups as Qualitative Research. $\left(2^{\text {nd }}\right.$ ed.). Thousand Oaks, CA: Sage Publications.

Morrison, K.A., Robbins, H.H., \& Gregory Rose, D. (2008). Operationalizing culturally relevant pedagogy: Synthesis of classroom based-research. Equity \& Excellence in Education, 41(4), 433-452.

National Center for Research on Cultural Diversity and Second Language Learning. (1994). Funds of knowledge: Learning from language minority households. Washington, DC: Author Available:www.cal.org/resources/Digest/ncreds01.html

Nieto, S., \& Bode, P. (2008). Affirming Diversity: The Sociopolitical Context of Multicultural Education (5 ${ }^{\text {th }}$ ed.). Boston: Pearson Education, Inc.

No Child Left Behind Act of 2001, Pub. L. No. 107-110. 115 Stat. 1425 (2002).

Pace, D. (2011). Best practice: The use of explicit instruction and culturally responsive teaching. Insights on Learning Disabilities, 8(2), 5-14.

Peck, S., \& Serrano, A.M. (2002). Open Court and English language learners: Questions and strategies. Paper presented at the Annual Meeting of the American Association for Applied Linguistics, Salt Lake City, UT, April 6-9.

Peralta-Nash, C. (2003). The impact of home visit in students' perceptions of teaching. Teacher Education Quarterly, 30(4), 111-125.

Powell, R. (1997). Then the beauty emerges: A longitudinal case study of culturally relevant teaching. Teaching and Teacher Education, 13(5), 467-484. 
Ravitch, D. (2010). The Death and Life of the Great American School System: How Testing and Choice are Undermining Education. New York: Perseus Books Group.

Saldaña, J. (2009). The Coding Manual for Qualitative Researchers. Los Angeles: Sage.

Schmidt P.R., \& Lazar, A.M. (Eds.). (2011). Practicing What We Teach: How Culturally Responsive Literacy Classrooms Make a Difference. New York: Teachers College Press.

Scripted reading instruction. (n.d.). In EdResearch Info online. Retrieved from http://edresearch.info/scripted.asp

Seidman, I. (2006). Interviewing as Qualitative Research: A Guide for Researchers in Education and the Social Sciences ( ${ }^{\text {rd }}$ ed.). New York: Teachers College Press.

Shannon, P. (1998). Awareness and attitudes toward literacy. In M.F. Optiz (Ed.), Literacy Instruction for Culturally and Linguistically Diverse Students. (213-228). Newark, DE: International Reading Association.

Singleton, G.E. \& Linton, C. (2006). Courageous Conversations About Race: A Field Guide for Achieving Equity in Schools. Thousand Oaks, CA: Corwin Press, Inc.

Sleeter, C.E. (2005). Un-Standardizing Curriculum: Multicultural Teaching in the Standards-Based Classroom. (J.A. Banks, Ed) New York: Teachers College Press.

Toppel, K. (2012). Phonics instruction with a culturally responsive twist: Three approaches to transforming curriculum. Multicultural Perspectives, 14(2), 1-4.

Yin, R.K. (2009). Case Study Research: Design and Methods (4 ${ }^{\text {th }}$ ed.). Los Angeles: Sage.

Veléz-Ibañez, C.G. (1988). Networks of exchange among Mexicans in the U.S. and Mexico: Local level Responses to national and international transformations. Urban Anthropology, 17(1), 27-51.

Veléz-Ibañez, C.G., \& Greenberg, J.B. (1992). Formation and transformation of funds of knowledge among U.S.-Mexican households. Anthropology \& Education Quarterly, 23(4), 313-335.

Vogt, M., \& Echevarría, J. (2008). 99 Ideas Activities for Teaching English Learners with The SIOP Model. Boston, MA: Pearson Education, Inc. 


\section{APPENDIX A \\ “A MOTIVATIONAL FRAMEWORK FOR CULTURALLY RELEVANT TEACHING" FRAMEWORK FROM CARE TRAINING}

\section{A. Establishing Inclusion: How does the learning experience contribute to developing as a community of learners who feel respected and connected to one another?}

Routines and rituals are visible and understood by all:

1. Rituals are in place that help African American and Latino students feel that they belong in class.

2. African American and Latino students and teacher(s) have opportunities to learn about each other.

3. African American and Latino students and teacher(s) have opportunities to learn about each other's unique backgrounds.

4. Classroom agreements and rules and consequences for violating agreements are negotiated.

5. The system of discipline is understood by all students and applied with fairness.

6. Teacher directs attention equitably for African American and Latino students.

7. Teacher interacts respectful with and according to African American and Latino students.

8. Teacher demonstrates that $\mathrm{s} / \mathrm{he}$ cares about African American and Latino students.

9. African American and Latino students talk to and with partners in small group work.

10. African American and Latino students have opportunities to respond to the lessons by writing or speaking.

\section{B. Developing a Positive Attitude: How does the learning experience offer meaningful choices and promote personal relevance to contribute to a positive attitude?}

Teacher works with African American and Latino students to personalize the relevance of course content:

1. African American and Latino students' experiences, concerns, and interests are used to develop course content.

2. African American and Latino students' experiences, concerns, and interests are addressed in response to questions. 
3. African American and Latino students' prior knowledge and learning experiences are explicitly linked to course content.

4. Teacher encourages African American and Latino students to understand, develop, and express different points of view.

5. Teacher encourages African American and Latino students to clarify their interests and set goals.

6. Teacher maintains flexibility in pursuit of "teachable moments" and emerging interests.

Teacher encourages African American and Latino students to make real choices such as:

7. how to learn

8. what to learn

9. when a learning experience will be considered complete

10. how learning will be addressed

\section{Enhancing Meaning: How does the learning experience engage participants in challenging learning?}

The teacher encourages all students to learn, apply, create, and communicate knowledge:

1. Teacher helps African American and Latino students to activate prior knowledge and to use it as a guide to learning.

2. Teacher, in concert with African American and Latino students, creates opportunities for inquiry, investigation, and projects.

3. Teacher provides opportunities for African American and Latino students to actively participate in challenging ways.

4. Teacher asks higher order questions of African American and Latino students throughout a lesson.

5. Teacher elicits high quality responses from African American and Latino students.

6. Teacher uses multiple "safety nets" to ensure African American and Latino student success.

\section{Engendering Competence: How does the learning experience create an understanding that participants are becoming more effective in learning they value and perceive as authentic to real world experiences?}

There is information, consequence, or product that supports African American and Latino students in valuing and identifying learning:

1. Teacher clearly communicates the purpose of the lesson. 
2. Teacher clearly communicates criteria for excellent final products.

3. Teacher provides opportunities for a diversity of competencies to be demonstrated in a variety of ways.

4. Teacher helps African American and Latino students to concretely identify accomplishments.

5. Teacher uses multiple forms of assessments.

6. Teacher assesses progress continually in order to provide feedback on individual growth and progress.

7. Teacher creates opportunities for African American and Latino students to make explicit connections between new and prior learning.

8. Teacher creates opportunities for African American and Latino students to make explicit connections between their learning and the "real world."

9. Teacher provides opportunities for African American and Latino students to self-assess learning in order to reflect on their growth as learners.

10. Teacher provides opportunities for African American and Latino students to self-assess their personal responsibility for contributing to the classroom as a learning community. 


\section{APPENDIX B}

\section{EMAIL COVER LETTER}

Dear CARE Team Member,

Because you have participated in CARE training this school year, I would like to invite you to be part of a study I will be conducting for my doctorate in Educational Leadership pending approval from Portland State University.

I was a member of Durham's CARE team this year and attending the trainings has sparked my interest in how teachers go about getting to know their culturally and linguistically diverse students and their families as well as how teachers who use scripted curricula experienced the process of creating culturally responsive lessons. I am looking for six elementary teachers to be part of my study, which involves 3 individual interviews and one focus group session.

I have attached a letter of informed consent, which provides additional information about the study. Please contact me via email or by phone by the end of this week if you are interested in participating in the study. I'd like to start interviews in July, so I will need summer contact info from interested teachers as I know district email may not be checked beyond this week. I am more than happy to answer any questions you may have about the study. My contact information is listed below.

Thank you for your consideration,

Katie

Katie Toppel

Kindergarten Teacher

Durham Elementary

District email: ktoppel@ttsd.k12.or.us

Home email: katie.toppel@gmail.com

Cell: 203-526-1245 


\section{APPENDIX C \\ LETTER OF INFORMED CONSENT}

\section{A Case Study of Teachers' Perceptions about the Interplay Between Culturally Responsive Instruction and Scripted Curricula}

You are invited to participate in a research study conducted by Katie Toppel who is a student at Portland State University pursuing a doctorate in Educational Leadership with a focus on Curriculum and Instruction. Katie is also a Kindergarten Teacher at Durham Elementary School where she is a CARE team member. The researcher hopes to learn more about what teachers do to better understand their culturally and linguistically diverse students and how teachers who use scripted curricula describe the process of creating culturally responsive lessons geared towards their culturally and linguistically diverse students. This study is being conducted in partial fulfillment of the requirements for a doctoral degree and will be conducted under the supervision of Dr. Samuel Henry who is a faculty member at Portland State University. You were selected as a possible participant in this study because you are a member of your schools' CARE team and participated in CARE trainings during the 2011-2012 school year.

If you decide to participate, you are being asked to participate in a series of three individual interviews with the researcher that will take place in July, August, and September. Each interview will last approximately 45-60 minutes during which time you will be asked to share your experiences pertaining to the creation of culturally responsive lessons as part of CARE training and to describe ways in which you have attempted to or have been successful in gaining a better understanding of your focal students.

Additionally, you are being asked to participate in one focus group session that will last approximately one hour to which you are encouraged to bring one additional colleague.

While participating in this study, it is possible that negative and/or positive experiences may trigger powerful emotions. You may not receive any direct benefit from taking part in this study, but you will experience opportunities to reflect on your own practice, furthering your personal and professional development. Any information that is obtained in connection with this study will be kept anonymous and confidential when possible.

Your participation is voluntary. You do not have to take part in this study, and it will not affect your relationship with Tigard-Tualatin School District, Portland State University, or Katie Toppel. If you choose to participate, please be aware that the focus group session will involve discussing topics and sharing opinions/ideas in a group setting, therefore this session will not be confidential. You may withdraw from the study at any time without affecting your relationship with the aforementioned institutions. 
If you have concerns or problems about your participation in this study or your rights as a research subject, please contact Human Subjects Research Review Committee, Office of Research and Strategic Partnerships, Market Center Building, $6^{\text {th }}$ Floor, Portland State University, (503) 725-4288. If you have questions about the study itself, contact Katie Toppel at Durham Elementary School, 7980 SW Durham Road, Tigard, OR 97224, (503) 431-4554 work / (203) 526-1245 cell / email ktoppel@tttsd.k12.or.us

Your signature indicates that you have read and understand the above information and agree to take part in this study. Please understand that you may withdraw your consent at any time without penalty, and that, by signing, you are not waiving any legal claims, rights or remedies. The researcher will provide you with a copy of this form for your own records.

Name 


\section{APPENDIX D}

\section{EMAIL REMINDER}

\section{Hello CARE participants!}

I know this is far from an ideal time to be asking you to think about anything but summer break, but I am still looking for 3 teachers to participate in my research study. I am particularly in need of teachers who teach grades 1-5. As you bask in the glory of your quiet classroom after all the kiddos leave for the summer, please consider taking part. I am very interested in hearing your perspectives about how you've been working with your culturally and linguistically diverse students and incorporating culturally responsive lessons into the structure of scripted curricula we utilize in TTSD.

Participation involves about 4 hours of your time, which includes 3 individual interviews and one focus group session spread out between July and September. In order to make participation as convenient for you as possible, I am willing to come to your home for interviews or a location close by to reduce your travel time.

Thank you for your consideration and please let me know if you are interested in becoming a participant. All I would need at this point is an email address/phone number I could use to contact you over the summer.

\section{Happy Summer!}

Katie

Katie Toppel

Kindergarten Teacher

(503) 431-4554 


\section{APPENDIX E}

\section{QUESTIONNAIRE TO DETERMINE MAXIMUM VARIATION AMONG SAMPLE POPULATION}

The following survey will be sent via Survey Monkey to teachers interested in participating if the number of interested teachers exceeds six. The data will later be used to determine maximum variance among participants with respect to grade level taught, gender, years of teaching experience, and availability during Summer 2012 to participate in the study.

1. Which elementary school do you work at?

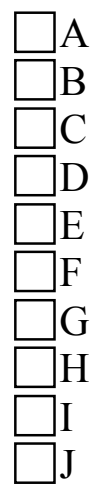

2. What grade/position do you teach?

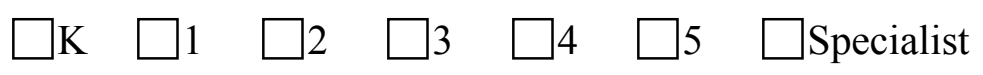

3. How many years of teaching experience do you have?

4. Have you ever conducted a home visit?

$\square$ Yes

$\square$ No

4a.If yes, approximately how many home visits have you done?

5. Will you be available in August of 2012 to participate in individual interviews?

$\square$ Yes

$\square$ No

6. What is your gender?

$\square$ Male

$\square$ Female 


\section{APPENDIX F}

\section{PROTOCOL FOR INDIVIDUAL INTERVIEWS}

Interviewee:

Interview \#:

Date \& Time \& Location:

1. Begin by sharing the following: Thank you for your willingness to participate in this project. Before we begin, I would like to reassure you that this interview will be confidential and the tape/transcripts available only to me, my academic advisers at PSU, and 5 other doctoral colleagues who are part of my cohort. Excerpts of this interview may be made part of the final research report and may be discussed with the aforementioned individuals, but under no circumstances will your name or identifying characteristics be included in the report. Please be aware there are no right or wrong answers. Is it all right for me to turn on the recorder now?

2. Remind participant of the purpose of the study:

- To understand ideas and behaviors teachers have developed as ways to better understand their culturally and linguistically diverse students

- To understand how teachers who use scripted curricula create culturally responsive lessons intended to specifically connect to their culturally and linguistically diverse students' funds of knowledge.

3. Consult calendars and confirm next individual interview.

Interview 1

June 2012
1. Tell me about your teaching experience.

2. Tell me about how/why you became part of your school's CARE team.

3. Tell me about your focal students and how/why you selected them.

4. How do you go about learning about students whose races or cultures differ from yours (your focal students)?

5. What barriers have you encountered in your efforts to collect below the line information about your focal students?

6. Can you share something particularly memorable that you learned about one of your focal students?

7. Tell me about what you think it means to be a culturally responsive teacher.

8. Do you consider yourself to be a culturally responsive teacher? Why or why not?

9. Gloria Ladson-Billings, Cultural Competency- Duration: $3: 10$ http://www.youtube.com/watch?v=ccEu7r2IwM0\&feature $=$ related

What, if anything, from Gloria's commentary resonates with you? 
Interview 2

July 2012

Interview

3

August.

2012
1. Explain to me how you go about planning what you will teach and how you will teach it.

2. What curricula do you use?

3. When you began to plan your culturally responsive lesson(s), what was your focus in terms of the four R's (Rigor, Relationships, Relevance, and Realness) from the Motivational Framework for Culturally Relevant Teaching and why?

4. Did your lesson draw upon the below the line information you collected about your focal students? If so, can you explain how the below the line information influenced your lesson?

5. Walk me through one of the culturally responsive lessons that you created.

6. To what extent did you follow and/or deviate from the curriculum purchased by the district in the process of creating your culturally responsive lesson and why?

7. Describe any challenges or successes you encountered in the process of creating your culturally responsive lesson plans.

8. Do you think the culturally responsive lessons had an impact on your focal students? If so, in what ways?

9. Tell me about an "aha" moment you experienced or something notable you learned in the process of creating culturally responsive lessons.

10. Do you think you will continue to attend to cultural responsiveness in your instruction? If so, how will you integrate culturally responsive lessons in with the curricula you already use?

1. Have you ever done a home visit?

2. What do you think about home visits?

3. Do you think home visits affect culturally and linguistically diverse students in the classroom? Why or why not?

4. Do you think elementary teachers should do home visits? Why or why not?

5. What might teachers gain from home visits that they couldn't access by interacting with a student or the student's family in the school setting?

6. Can you describe a memorable experience you had when visiting a student's home? 


\section{APPENDIX G}

\section{PROTOCOL FOR FOCUS GROUP CONVERSATION}

Date \& Time \& Location:

Members Present:

1. Begin by sharing the following: Thank you for your willingness to participate in this project. Before we begin, I would like to reassure you that this discussion will be confidential from others not in participation and the tape/transcripts available only to me, my academic advisers at PSU, and 5 other doctoral colleagues who are part of my cohort. Bear in mind however that participants in attendance will know your identity and hear your comments today. Excerpts of this interview may be made part of the final research report and may be discussed with the aforementioned individuals, but under no circumstances will your name or identifying characteristics be included in the report.

Please be aware there are no right or wrong answers. Is it all right for me to turn on the recorder and video now?

2. Remind participants of the purpose of the study:

- To understand ideas and behaviors teachers have developed as ways to better understand their culturally and linguistically diverse students

- To understand how teachers who use scripted curricula create culturally responsive lessons intended to specifically connect to their culturally and linguistically diverse students.

3. Distribute paper/pens for notes/free write.

Focus Group Conversation

August or September 2012

\begin{tabular}{l|l} 
Part 1 & Participants will first view a YouTube video and will be asked to keep home
\end{tabular} visits in mind as they watch it.

[Teaching Diverse Students Initiative-Culturally Responsive Instruction, Duration 4:40

http://www.youtube.com/watch?v=nGTVjJuRaZ8\&feature=related]

\begin{tabular}{l|l} 
Part 2 & 5 minute free write to allow participants time to capture ideas and thoughts
\end{tabular} prompted by the video (artifact for data analysis)

1. How would you describe the connection between home visits and culturally responsive instruction?

2. Let's brainstorm some of the benefits and challenges associated with home visits. (artifact for data analysis).

3. Can anyone share a personal experience with visiting a student at home?

4. Do you have any ideas about how teachers should determine which homes to visit?

5. Do you have any ideas about how teachers should go about initiating home visits with families? 


\section{APPENDIX H}

\section{DATA COLLECTION TIMELINE}

Plan A-Revised

\begin{tabular}{|l|l|}
\hline \multicolumn{1}{|c|}{ Data Collection } & \multicolumn{1}{c|}{ Schedule } \\
\hline In depth interviews-Round 1 & June 2012 \\
\hline In depth interviews-Round 2 & July 2012 \\
\hline Focus Group & August 2012 \\
\hline In depth interviews-Round 3 & End of August 2012 \\
\hline \multicolumn{1}{|c|}{ Data Analysis } & $\begin{array}{l}\text { On going throughout data collection and } \\
\text { following round 3 of interviews in } \\
\text { September, October, November, and } \\
\text { December }\end{array}$ \\
\hline
\end{tabular}

Plan B

\begin{tabular}{|l|l|}
\hline \multicolumn{1}{|c|}{ Data Collection } & \multicolumn{1}{c|}{ Schedule } \\
\hline In depth interviews-Round 1 & August 2012 \\
\hline In depth interviews-Round 2 & September 2012 \\
\hline Focus Group & End of September 2012 \\
\hline In depth interviews-Round 3 & October 2012 \\
\hline \multicolumn{1}{|c|}{ Data Analysis } & $\begin{array}{l}\text { On going throughout data collection and } \\
\text { following round 3 of interviews in } \\
\text { November, December, and January }\end{array}$ \\
\hline
\end{tabular}

